WSRC-TR-2000-00205

KEYWORDS:

Mixer Model

CFD Approach

Mixer Shroud Model

Propeller Model

Asymmetric Wall Jet Model

RETENTION - Permanent

\title{
Three-Dimensional CFD FLYGT Mixer Model and Results
}

SAVANNAH RIVER TECHNOLOGY CENTER

Si Young Lee and Richard A. Dimenna

Publication Date: June, 2000

Westinghouse Savannah River Company

Savannah River Site

Aiken, SC 29808

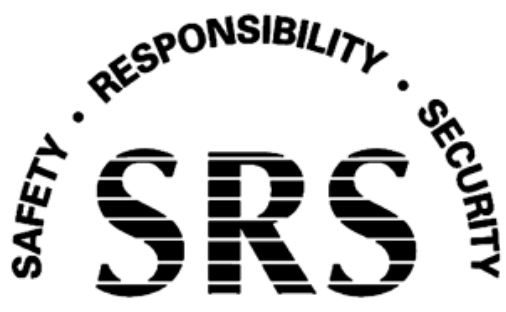

SAVANNAH RIVER SITE

Prepared for the U.S. Department of Energy under Contract No. DE-AC09-96SR18500 
This document was prepared in conjunction with work accomplished under Contract No. DE-AC09-96SR18500 with the U. S. Department of Energy.

\section{DISCLAIMER}

This report was prepared as an account of work sponsored by an agency of the United States Government. Neither the United States Government nor any agency thereof, nor any of their employees, makes any warranty, express or implied, or assumes any legal liability or responsibility for the accuracy, completeness, or usefulness of any information, apparatus, product or process disclosed, or represents that its use would not infringe privately owned rights. Reference herein to any specific commercial product, process or service by trade name, trademark, manufacturer, or otherwise does not necessarily constitute or imply its endorsement, recommendation, or favoring by the United States Government or any agency thereof. The views and opinions of authors expressed herein do not necessarily state or reflect those of the United States Government or any agency thereof.

This report has been reproduced directly from the best available copy.

Available for sale to the public, in paper, from: U.S. Department of Commerce, National Technical Information Service, 5285 Port Royal Road, Springfield, VA 22161, phone: (800) 553-6847, fax: (703) 605-6900

email: orders@ ntis.fedworld.gov

online ordering: http://www.ntis.gov/support/index.html

Available electronically at http://www.osti.gov/bridge

Available for a processing fee to U.S. Department of Energy and its contractors, in paper, from: U.S. Department of Energy, Office of Scientific and Technical Information, P.O. Box 62, Oak Ridge, TN 37831-0062,

phone: (865)576-8401,

fax: (865)576-5728

email: $\underline{\text { reports@ adonis.osti.gov }}$ 
(This Page Intentionally Left Blank) 
DOCUMENT: WSRC-TR-2000-00205

TITLE: Three-Dimensional CFD FLYGT Mixer Model and Results

\section{APPROVALS}

Date:

Si Y. Lee, Author (EM\&S Group/EDS/SRTC)

Date:

Richard A. Dimenna, Coauthor (EM\&S Group/EDS/SRTC)

Date:

Glenn A. Taylor, Technical Reviewer (Process Engineering)

Date:

Cynthia P. Holding-Smith, Manager (EM\&S Group/SRTC)

Date:

Martha A. Ebra, Manager (EDS/SRTC)

Date:

Brannen J. Adkins, Customer (Waste Removal Engineering) 
(This Page Intentionally Left Blank) 


\section{Table of Contents}

$\begin{array}{ll}\text { Summary } & 1\end{array}$

1. Background and Objective 2

2. Model Descriptions and Solution Methods 3

3. Investigation of the Nominal Pump Operating Conditions 12

4. Results and Discussions $\quad 20$

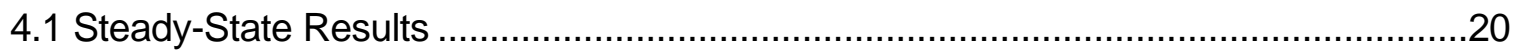

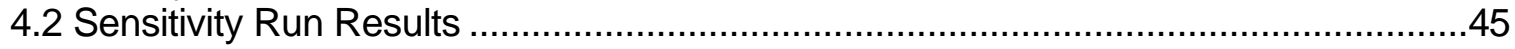

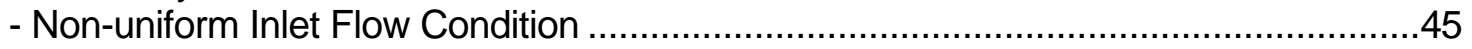

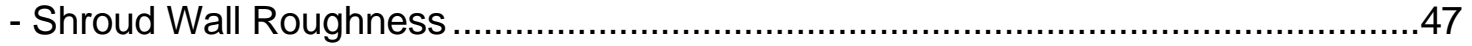

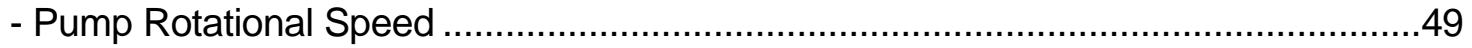

4.3 Transient Flow Behavior of FLYGT Mixer Associated with Pump Cavitation ...............51

5. Conclusions $\quad 63$

6. Recommendations $\quad 64$

$\begin{array}{ll}\text { 7. References } & 65\end{array}$ 
(This Page Intentionally Left Blank) 


\section{List of Figures}

Figure 1. Analysis methodology for FLYGT mixer models ............................................

Figure 2. Modeling boundary for three-dimensional FLYGT mixer model ............................7

Figure 3. Meshes on three-dimensional domain .........................................................

Figure 4. The blade and shaft surface grid after two repetitions of periodic boundary........10

Figure 5. Two-dimensional meshes at exit plane after two periodic repetitions of the present model ......................................................................................11

Figure 6. Overview of segregated solution method for the present model........................12

Figure 7. Comparison of predictions with test data ……................................................14

Figure 8. Determination of pump operating conditions ...................................................15

Figure 9. Axial velocity distribution as a function of liquid level at the $40 \mathrm{ft}$ distance from the shroud exit based on two-dimensional wall jet model (Lee and Dimenna, 2000) for $9000 \mathrm{gpm}$ pump flowrate. Inlet velocity is uniformly $45^{\circ}$ skewed against the axial flow direction $\left(\theta_{\text {in }}=45^{\circ}\right.$ in the figure).

Figure 10. Axial velocity distributions as a function of liquid depth at various distances distance from the shroud exit based on two-dimensional wall jet model (Lee and Dimenna, 2000) for 9,000 gpm pump flowrate. Inlet velocity is increased linearly along the radial direction.

Figure 11. Velocity vector plot near the shroud region predicted by the two-dimensional wall jet model (Lee and Dimenna, 2000) for 9,000 gpm pump flowrate. Inlet velocity is increased linearly along the radial direction.

Figure 12. Velocity distributions near the shroud region and over the whole computational domain predicted by two-dimensional wall jet model (Lee and Dimenna, 2000) for 9,000 gpm pump flowrate. Inlet velocity is increased linearly along the radial direction.

Figure 13. Typical static pressure distribution at downstream and upstream regions near the leading-edge tip of single pump blade for 860rpm of pump rotational speed and 9,000 gpm flow condition (Propeller blade shown in the figure rotates clockwise.).

Figure 14. Typical fluid flow pattern and rotation profile at downstream and upstream regions near the leading-edge tip of single pump blade for 860rpm of pump 
rotational speed and 9,000 gpm flow condition (Propeller blade shown in the figure rotates clockwise.).

Figure 15. Load on each component of the FLYGT mixer along the axial flow direction for 860rpm of pump rotational speed (Loads for propeller and shroud wall region are negative forces).

Figure 16. Radial velocity vector plots at the plane crossing the leading tip of the blade for various rotational speeds of $9,000 \mathrm{gpm}$ pump flowrate.

Figure 17. Static pressure contour plots at the plane crossing the leading tip of the blade for various rotational speeds of 9,000 gpm pump flowrate.

Figure 18. Vorticity contour plots at the plane crossing the leading tip of the blade for various rotational speeds of 9,000 gpm pump flowrate.

Figure 19. Pressure distributions along the axial flow direction (line A-A') crossing the leading-edge tip of the propeller blade for $9,000 \mathrm{gpm}$ flow condition as a function of pump speed.

Figure 20. Fluid vorticity distributions along the axial flow direction (line A-A') crossing the leading-edge tip of the propeller blade for $9,000 \mathrm{gpm}$ flow condition as a function of pump speed e.

Figure 21. Turbulence intensity contour plots at the plane crossing the leading tip of the blade for various rotational speeds of 9,000 gpm pump flowrate.

Figure 22. Static pressure contour plot on the propeller surface.

Figure 23. Shear stress contour plot on the propeller surface. 35

Figure 24. Velocity contour plot near the pump propeller. 36

Figure 25. Vorticity contour plot at the pump propeller region (counterclockwise propeller direction).

Figure 26. Vorticity contour plot at the propeller hub region (counterclockwise propeller direction).

Figure 27. Static pressure contour plot at the propeller hub region (counterclockwise propeller direction)

Figure 28. Shear stress contour plot at the propeller hub region (counterclockwise propeller direction)

Figure 29. Static pressure contour plot at the shroud wall (counterclockwise propeller direction).

Figure 30. Shear stress contour plot at the shroud wall (counterclockwise propeller direction).

$$
\text { -viii- }
$$


Figure 31. Velocity distributions along the radial direction.

Figure 32. Velocity vector plot at the inlet and exit planes of shroud exit.........

Figure 33. Non-uniform and uniform flow conditions at the pump inlet for the sensitivity runs.

Figure 34. Sensitivity run results for axial flow velocities at shroud exit. .46

Figure 35. Velocity vector plot at the inlet and exit planes for non-uniform inlet flow condition.

Figure 36. Sensitivity run results for surface roughness effects of shroud wall on axial flow velocities at shroud exit (Roughness height of rough wall used in this figure was $0.01 \mathrm{ft}$ equivalent to concrete surface.).

Figure 37. Sensitivity run results for pump speed effects on axial flow velocities at the exit of the pump shroud under $9,000 \mathrm{gpm}$ flow and $860 \mathrm{rpm}$ pump speed.

Figure 38. Transient response of flow velocity along the radial direction of the shroud exit plane for $12,000 \mathrm{gpm}$ and $860 \mathrm{rpm}$ operating condition.

Figure 39. Transient response of axial flow velocity along the radial direction of the shroud exit plane for $12,000 \mathrm{gpm}$ and $860 \mathrm{rpm}$ operating condition.

Figure 40. Transient response of total pressure force for three propeller blades under $12,000 \mathrm{gpm}$ and $860 \mathrm{rpm}$ operating condition.

Figure 41. Transient response of pressure force for hub under $12,000 \mathrm{gpm}$ and $860 \mathrm{rpm}$ operating condition.

Figure 42. Transient response of pressure force for shroud wall under 12,000 gpm and 860 rpm operating condition.

Figure 43. Transient response of static pressure at leading-tip edge plane of the propeller.

Figure 44. Transient response of fluid density at leading edge plane of the propeller tip....59

Figure 45. Transient response of fluid velocity at leading edge plane of the propeller tip. ..60

Figure 46. Transient response of fluid vorticity at leading edge plane of the propeller tip...61

Figure 47. Transient velocity distributions at the exit of the shroud for $700 \mathrm{rpm}$ pump speed and $12000 \mathrm{gpm}$ flow condition..

Figure 48. Transient response of total pressure force for three propeller blades under $12000 \mathrm{gpm}$ flow with two pump speeds.. 
(This Page Intentionally Left Blank) 


\section{List of Tables}

Table 1. Modeling purpose and descriptions for Tank 19 FLYGT mixer..........................35

Table 2. Potential ranges of flow conditions for Tank 19 FLYGT mixer............................8

Table 3. Absolute pressures near the leading-edge tip of the pump blade under 9,000 gpm water flowrate of steady-state operating condition.

Table 4. Maximum axial and radial velocities for various pump speeds at the leading-edge tip of the pump blade under 9,000 gpm flow constraint of steady-state operating condition.

Table 5. Axial forces along the flow direction for propeller, hub, and shroud regions under wide range of steady-state pump operating conditions.

Table 6. Comparison of minimum pressure coefficients $\left(C_{p, m i n}\right)$ for three different advance coefficients $(\mathrm{J})$ corresponding to three rotational pump speeds.

Table 7. Influence of inlet flow non-uniformity on the axial loads of pump blade, shaft, and shroud wall regions under steady-state operating conditions of two different flows with $860 \mathrm{rpm}$ pump speed.

Table 8. Influence of shroud wall roughness on the axial loads of pump blade, shaft, and shroud wall regions under 9,000 gpm flow and $860 \mathrm{rpm}$ pump speed of steadystate operating condition.

Table 9. Influence of rotational pump speed on the axial loads of pump blade, shaft, and shroud wall regions under $9,000 \mathrm{gpm}$ flow of steady-state operating condition in a smooth shroud wall.

Table 10. Summary of load sensitivities to the changes of the physical parameters for the propeller blade for $9,000 \mathrm{gpm}$ flow and $860 \mathrm{rpm}$ operating condition (Negative number means decrease in load.). 
(This Page Intentionally Left Blank) 


\section{Summary}

Since mid-February, the Engineering Development Section (EDS) has been assisting the High Level Waste (HLW) customer to assess the failure causes of the FLYGT mixer to be used to recover waste from Tank 19 and to redesign the mixer.

In support of this project, two Computational Fluid Dynamics (CFD) models have been developed. They are a steady-state pump model and a transient model considering the effects of vapor formation due to pump cavitation. The commercial CFD code, FLUENT, is used in the present analysis. The computations are performed for a three-dimensional model of the FLYGT mixer with the prototypic propeller shape. In the models, the propeller is assumed to be symmetrical and perfectly-balanced within a rigid and stationary shroud boundary under any operating condition. Both of the models include the propeller region, the inlet region upstream of the propeller, and the downstream shroud region as computational domain.

The areas of focus for the analysis presented here are the flow performance of the modified mixer under potential operating conditions and the loads on the propeller, shaft, and shroud regions of the mixer. Calculated physical parameters include velocity profiles of axial flow, cavitation locations, and loads on the shroud wall, propeller blade, and hub of the propeller for the operating flow conditions. As inlet boundary conditions of the model, uniform radial and axial velocities through the shroud inlet and around the motor housing are used assuming azimuthally symmetrical flow conditions.

An operating flow condition of $9,000 \mathrm{gpm}$ is established by the comparison of the steadystate model predictions and HLW test data (Adkins, 2000) at the shroud exit. The results of the model show that the modified mixer has reasonable performance for $9,000 \mathrm{gpm}$ flow and 500 rpm pump speed in terms of flow behavior and spread angle near the exit.

Sensitivity runs are performed for non-uniform inlet velocity along the axial flow direction, different surface roughness of the shroud inner wall, and different pump speeds. From the model results, the non-uniform inlet velocity and wall roughness effects are found to have a negligible effect on overall flow patterns at the inner and outlet regions of the shroud, loads on each pump component, and cavitation size near the tip of the blade as long as liquid flow rate remains constant. The primary reason for these results is that turbulent flow effect within the shroud is dominant due to the high-speed rotations of axial propeller. Thus, it is noted that pump speed is the most sensitive one among the three parameters in terms of flow performance and pump loading.

Transient model results show that transient flow behavior such as change of fluid density and loadings of the mixer establish steady-state conditions within about 1 second, corresponding to about 40 cycles of the propeller blade, after the initiation of the pump cavitation. In addition, a low density region is established near the peripheral region adjacent to the shroud wall because all bubbles generated as a result of cavitation migrate to the high-velocity fluid region near the tip of the propeller blade. 
Date:

\section{Background and Objective}

The Engineering Modeling and Simulation Group (EMSG) was asked to perform an analysis which could provide pertinent information in support of a modified High-Level Waste (HLW) Tank 19 FLYGT mixer design. The modified FLYGT mixer will be used to recover waste from the Tank 19. Under normal operating conditions, the mixer will be oriented in a horizontal position above approximately 6 to 7 inches above the bottom of the $85 \mathrm{ft}$ diameter tank. In this situation, the discharge from the shroud exit is the driving force for liquid mixing and material suspension from the tank floor.

In previous work (Lee and Dimenna, 2000), two two-dimensional models were developed to determine the optimum shroud length for acceptable flow performance and to conduct sensitivity analysis for a wide range of possible boundary conditions at the shroud inlet downstream of the pump propeller. One model was axisymmetric using the shroud alone without the pump propeller as a modeling boundary. The other model was a twodimensional wall jet model to investigate asymmetric effects of the shroud discharge flow.

For the previous analysis (Ref. 8) and the present work, a Computational Fluid Dynamics (CFD) approach has been taken. In the present analysis, a three-dimensional steadystate pump model and a transient cavitation model have been developed to evaluate detailed flow performance of the Tank 19 FLYGT mixer improved by the previous work. The commercial CFD preprocessor and solver, GAMBIT and FLUENT5.3, have been used to model the mixer. The geometry files of the mixer models have been created with the integrated CFD preprocessor GAMBIT based on the propeller model. At the beginning of April, Fluent Inc. was contracted to assist in the development of the propeller model based on digital propeller shape data obtained from Michigan Wheel Company, who manufactured the present propeller.

The present three-dimensional CFD models can identify a number of viscous flow phenomena in the propeller flow field confined in the shroud region, including blade and hub boundary layers, flow separation on the blade, hub and tip vortices, etc. Thus, the models help estimate reasonable loading conditions for the optimum operating performance of the mixer. Parametric sensitivity studies with the models also help identify operation and design variations that may be used to improve the operational design of the mixer from the aspect of pump flow performance. The main objectives of the present work are as follows:

1. To examine detailed flow performance of the Tank 19 FLYGT mixer improved by the previous work,

2. To conduct sensitivity analysis for a wide range of possible boundary conditions, and

3. To investigate transient flow behavior and loading of the FLYGT mixer.

For the present study, a flow simulation method is developed to calculate the flow around a marine-type propeller configuration of the FLYGT mixer. The flow characteristics of the blade boundary layer and tip vortex are investigated by the CFD method. Comparisons between calculation results and experimental data are made to demonstrate the capability of the model to handle propeller flows. The analysis results for the mixer model provide 
design guidance concerning the operation performance of a modified mixer, and this estimated loading information would be used as input to assess the structural vibration and stress analysis of the FLYGT mixer.

Table 1. Modeling purpose and descriptions for Tank 19 FLYGT mixer.

\begin{tabular}{|c|c|c|c|c|}
\hline \multicolumn{2}{|c|}{ Model } & Brief description & Primary purpose & Status \\
\hline \multicolumn{2}{|c|}{$\begin{array}{c}\text { Axisymmetric 2-D } \\
\text { model }\end{array}$} & $\begin{array}{l}\text { Shroud model using } \\
\text { two-dimensional CFD } \\
\text { approach }\end{array}$ & $\begin{array}{l}\text { To assess the impact of shroud } \\
\text { length on discharge flow } \\
\text { behavior for various } \\
\text { boundary conditions }\end{array}$ & $\begin{array}{c}\text { Done } \\
\text { (Ref. 8) }\end{array}$ \\
\hline \multicolumn{2}{|c|}{ 2-D wall jet model } & $\begin{array}{l}\text { Two-dimensional wall } \\
\text { jet model with the } \\
\text { pump shroud } \\
\text { submerged in } 4 \mathrm{ft} \text { tank } \\
\text { level }\end{array}$ & $\begin{array}{l}\text { To investigate asymmetric } \\
\text { effect of the liquid discharge } \\
\text { from the optimum length of } \\
\text { shroud due to the presence } \\
\text { of the tank bottom }\end{array}$ & $\begin{array}{c}\text { Done } \\
\text { (Ref. 8) }\end{array}$ \\
\hline \multirow[t]{2}{*}{$\begin{array}{l}\text { 3-D } \\
\text { FLYGT } \\
\text { mixer } \\
\text { model }\end{array}$} & $\begin{array}{l}\text { Steady- } \\
\text { state } \\
\text { model }\end{array}$ & $\begin{array}{l}\text { Detailed three- } \\
\text { dimensional pump } \\
\text { model with prototypic } \\
\text { propeller blade shape } \\
\text { to exclude cavitation } \\
\text { model }\end{array}$ & $\begin{array}{l}\text { To assess pump flow } \\
\text { performance in detail and to } \\
\text { evaluate load on propeller } \\
\text { blade, hub, and pump } \\
\text { shroud in a steady-state } \\
\text { operation }\end{array}$ & $\begin{array}{l}\text { Present } \\
\text { analysis }\end{array}$ \\
\hline & $\begin{array}{c}\text { Transient } \\
\text { model }\end{array}$ & $\begin{array}{l}\text { Detailed three- } \\
\text { dimensional pump } \\
\text { model with prototypic } \\
\text { propeller blade shape } \\
\text { to include cavitation } \\
\text { model }\end{array}$ & $\begin{array}{l}\text { To evaluate transient load on } \\
\text { propeller blade, hub, and } \\
\text { pump shroud and to } \\
\text { estimate transient time to } \\
\text { reach steady-state mode }\end{array}$ & $\begin{array}{l}\text { Present } \\
\text { analysis }\end{array}$ \\
\hline
\end{tabular}

\section{Model Descriptions and Solution Methods}

Two CFD models have been developed to examine the flow performance of the modified Tank 19 FLYGT mixer (Lee and Dimenna, 2000) and to conduct sensitivity analysis for a wide range of possible boundary conditions. The modeling purpose and brief descriptions for the models are provided in Table 1. The methodology used for the models and analysis of the FLYGT mixer are shown in Fig. 1. The computational domain for the mixer model consists of three major regions, which are the propeller region, the inlet region upstream of the propeller, and the shroud region downstream of the propeller. The present modeling boundary is shown in Fig. 2.

For computational efficiency, a $120^{\circ}$ sector of the mixer was used as a computational domain by using the following assumptions:

- $\quad$ The blades of the propeller are perfectly balanced and symmetrical. 
Date: $04 / 22 / 02$

- The clearance gap between the tip of the blade and the shroud wall is kept constant.

- The inlet geometry is symmetrical in terms of geometry and flow distribution in an azimuthal direction.

- The flow distribution around the FLYGT mixer is fully mixed so that the wall boundary of the tank bottom has no impact on flow pattern just outside the shroud wall.

About 120,000 mesh nodes were established from the mesh sensitivity analysis on a Silicon Graphics, Inc. (SGI) workstation platform. The computational meshes on a threedimensional domain are shown in Fig. 3. It should be noted that both curved-side surfaces of the $120^{\circ}$ model were used as periodic boundaries for a rotational reference frame. Two repetitions of the periodic boundaries result in a complete FLYGT mixer as shown in Figs. 4 and 5 . For better convergence in numerical iterations, hexahedral meshes and smooth propagation of mesh size were adapted along the fluid-stream direction as shown in Fig. 5. The models assume isothermal conditions fixed at room temperature; the thermal effect on the performance of the pump is not calculated.

One of the two models is a steady-state pump model assuming the fluid is incompressible and is not affected by cavitation. The other one is a transient cavitation model to investigate effects of density change due to the cavitating fluid for high-speed rotation of propeller. As the fluid jet emerges from the tip of propeller blade, the boundary layer separates at the cavity created near the leading-edge of the blade. Then, it rolls up and produces the cavitation cloud. Reynolds number for one typical flow (corresponding to about $9,000 \mathrm{gpm}$ ) of potential conditions, as shown in Table 2, is about $1.4 \times 10^{6}$ based on shroud diameter $(D=0.5048 \mathrm{~m})$ and water at room temperature. That is,

$$
\mathrm{Re}=\frac{D \rho u}{\mu}=1.4 \times 10^{6}
$$

The flow condition is in the fully-turbulent flow region. A standard k- $\varepsilon$ turbulent model, namely, a two-equation model, was used for the present analysis. A velocity boundary condition was used at the shroud inlet. At the exit, a pressure boundary condition was used to determine flow pattern. The wall boundary of the shroud region used a no-slip boundary condition.

The steady-state model used mass continuity and three standard momentum equations with addition of Coriolis force to the convection term considering the rotating reference frame. In this situtation, relative velocity becomes

$\vec{v}_{r}=\vec{V}-\vec{\Omega} \times \vec{r}$

where

$\vec{\Omega}=$ angular velocity of the rotating frame and

$\vec{r}=$ position vector in the rotating frame.

After some manipulation of the standard Navier-Stokes equation using equation (2), the convection term $(\nabla \bullet(\rho \vec{v} \vec{v}))$ on the left-hand side of the momentum equation in a rotating frame of reference becomes 


$$
\nabla \bullet\left(\rho \vec{v}_{r} \vec{v}_{r}\right)+2 \rho \vec{\Omega} \times \vec{v}_{r}+\rho \vec{\Omega} \times(\vec{\Omega} \times \vec{r})
$$

Equation (3) shows that two new terms, referred to as Coriolis force and centrifugal force, respectively, in the literature, are added to the standard convection term for the rotational effect.

The transient cavitation model relies on the volume fraction concept so that it allows the fluids to be interpenetrating. The volume fraction of vapor phase created from the cavitation can therefore be equal to any value between 0 and 1, depending on the space occupied by vapor and liquid phases. The cavitation model allows mass to be transferred from one phase to another. This allows for modeling the formation of vapor from a liquid when fluid is cavitated. The model assumes no collapses of bubbles inside the shroud region and no slip between the two phases of the fluid because bubble residence time within the shroud is very short and bubbly flow regime is assumed to be dominant in the modeling domain. Thus, FLUENT solves a single-phase momentum equation for both phases and a volume fraction equation for the second phase. The volume fraction for the secondary vapor phase is determined from the continuity relation with interphase mass transfer from liquid to vapor phases. To get interphase mass transfer term, the change in bubble radius can be computed by a simplified Rayleigh equation assuming that the pressure within the bubble remains nearly constant when cavitation bubbles form in a liquid at room temperature. The latent heat of vaporization is neglected in isothermal cavitating flow. Bubble growth due to inertia effects is assumed negligible compared to caviation pressure. In this situation, bubble radius $R$ at time $t$ is determined by the following equation (Kubota et al., 1992):

$$
\frac{d R}{d t}=\left(\frac{2\left(p_{g}-p\right)}{3 \rho_{f}}\right)^{0.5}
$$

where $p_{g}$ is vapor pressure corresponding to fluid temperature and $\rho_{f}$ is the density of the liquid.

The total vapor mass $\left(m_{g}\right)$ can be written as

$$
m_{g}=\rho_{g}\left(\frac{4}{3} \pi R^{3}\right) n
$$

where $n$ is the number of bubbles per unit volume.

Bubble number density ( $n$ ) in eq. (5) was assumed to be constant. For the present work, $n$ was chosen as $1.0 \times 10^{4} \mathrm{~m}^{-3}$ from the literature (Kubota el al. (1992)). After some algebraic manipulation of eq. (5), rate of vapor formation due to cavitation can be written in terms of void fraction $\left(\alpha_{g}\right)$ as 


$$
\begin{aligned}
\frac{d m_{g}}{d t} & =\left(\frac{3 \rho_{g}}{R}\right)\left(\frac{4 \pi R^{3}}{3} n\right)\left(\frac{d R}{d t}\right) \\
& =\left(\frac{3 \rho_{g} \alpha_{g}}{R}\right)\left(\frac{d R}{d t}\right)
\end{aligned}
$$

Thus, an equation for the interphase mass transfer $\left(\mathrm{m}_{\mathrm{fg}}\right)$ due to cavitation can be obtained from eqs. (4) and (6). That is,

$$
m_{f g}=\frac{d m_{g}}{d t}=\left(\frac{3 \rho_{g} \alpha_{g}}{R}\right)\left(\frac{2\left(p_{g}-p\right)}{3 \rho_{f}}\right)^{0.5}
$$

Equation (7) is used in the continuity equation for the vapor phase, which is created from the cavitation phenomenon of the high-speed mixing pump.

Computations use the FLUENT rotating reference frame assumption in which the threedimensional flow field rotates in a reference frame that is fixed on the propeller blade. When equations of motion are solved in the steady-rotating reference frame, the Coriolis force term is added to the fluid acceleration term in the left-hand side of the ReynoldsAveraged Navier-Stokes equations derived in the inertial frame. The three-dimensional incompressible Navier-Stokes flow solver developed by Fluent, Inc. is applied to compute the rotating propeller flow in Tank 19 FLYGT mixer. The FLUENT solver is based on segregated solution method as shown in Fig. 6.

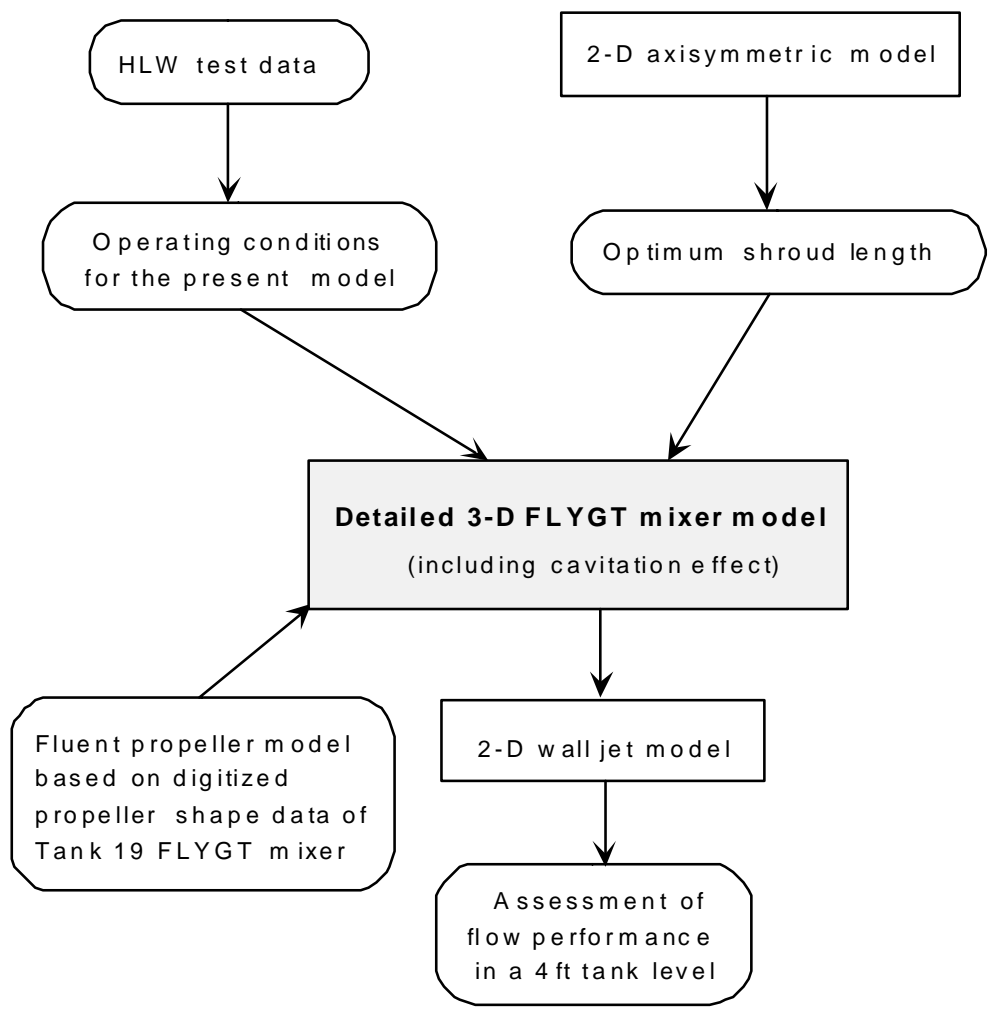

Figure 1. Analysis methodology for FLYGT mixer models 


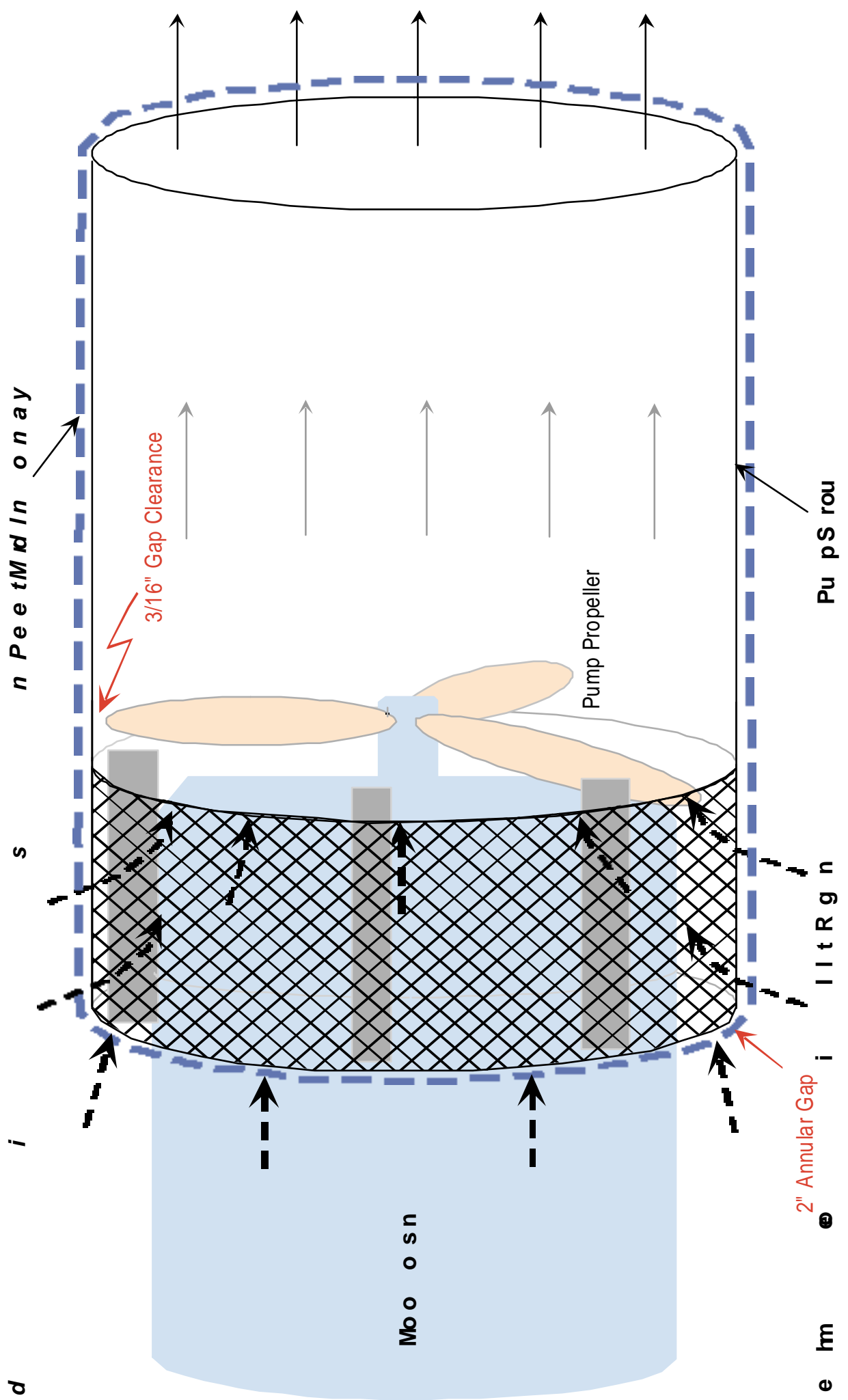

Figure 2. Modeling boundary for three-dimensional FLYGT mixer model o)

$-$ 
Table 2. Potential ranges of flow conditions for Tank 19 FLYGT mixer

\begin{tabular}{|c|c|c|c|c|c|c|c|c|}
\hline \multirow{2}{*}{$\begin{array}{l}\text { Flowrate } \\
\text { (gpm) }\end{array}$} & \multirow{2}{*}{$\begin{array}{r}\text { Flowrate } \\
\left(\mathrm{m}^{3} / \mathrm{sec}\right)\end{array}$} & \multirow{2}{*}{$\begin{array}{c}\text { Mass flow } \\
(\mathrm{kg} / \mathrm{sec})\end{array}$} & \multicolumn{2}{|c|}{ Inlet flow area } & \multirow{2}{*}{$\begin{array}{c}\text { Total flow area } \\
\left(\mathrm{m}^{2}\right)\end{array}$} & \multirow{2}{*}{\begin{tabular}{|c} 
Inlet velocity \\
$(\mathrm{m} / \mathrm{sec})$
\end{tabular}} & \multicolumn{2}{|c|}{ Average Velocity* } \\
\hline & & & Radial $\left(\mathrm{m}^{2}\right)$ & annular $\left(\mathrm{m}^{2}\right)$ & & & $(\mathrm{m} / \mathrm{sec})$ & $(\mathrm{ft} / \mathrm{sec})$ \\
\hline 9000 & 0.56781 & 566.674 & 0.48340 & 0.07842 & 0.56182 & 1.011 & 2.8368 & 9.3071 \\
\hline 10000 & 0.63090 & 629.638 & 0.48340 & 0.07842 & 0.56182 & 1.123 & 3.1520 & 10.3413 \\
\hline 11000 & 0.69399 & 692.602 & 0.48340 & 0.07842 & 0.56182 & 1.235 & 3.4672 & 11.3754 \\
\hline 12000 & 0.75708 & 755.566 & 0.48340 & 0.07842 & 0.56182 & 1.348 & 3.7824 & 12.4095 \\
\hline 12500 & 0.78863 & 787.048 & 0.48340 & 0.07842 & 0.56182 & 1.404 & 3.9400 & 12.9266 \\
\hline 13000 & 0.82017 & 818.530 & 0.48340 & 0.07842 & 0.56182 & 1.460 & 4.0976 & 13.4437 \\
\hline 13500 & 0.85172 & 850.012 & 0.48340 & 0.07842 & 0.56182 & 1.516 & 4.2552 & 13.9607 \\
\hline 14000 & 0.88326 & 881.493 & 0.48340 & 0.07842 & 0.56182 & 1.572 & 4.4128 & 14.4778 \\
\hline 14500 & 0.91481 & 912.975 & 0.48340 & 0.07842 & 0.56182 & 1.628 & 4.5704 & 14.9948 \\
\hline 15000 & 0.94635 & 944.457 & 0.48340 & 0.07842 & 0.56182 & 1.684 & 4.7280 & 15.5119 \\
\hline 15500 & 0.97790 & 975.939 & 0.48340 & 0.07842 & 0.56182 & 1.741 & 4.8856 & 16.0290 \\
\hline 16000 & 1.00944 & 1007.421 & 0.48340 & 0.07842 & 0.56182 & 1.797 & 5.0432 & 16.5460 \\
\hline 16500 & 1.04099 & 1038.903 & 0.48340 & 0.07842 & 0.56182 & 1.853 & 5.2008 & 17.0631 \\
\hline 17000 & 1.07253 & 1070.385 & 0.48340 & 0.07842 & 0.56182 & 1.909 & 5.3584 & 17.5802 \\
\hline 18000 & 1.13562 & 1133.349 & 0.48340 & 0.07842 & 0.56182 & 2.021 & 5.6736 & 18.6143 \\
\hline 19000 & 1.19871 & 1196.313 & 0.48340 & 0.07842 & 0.56182 & 2.134 & 5.9888 & 19.6484 \\
\hline
\end{tabular}

Note: * Average velocity was based on the cross-sectional flow area of the shroud. 


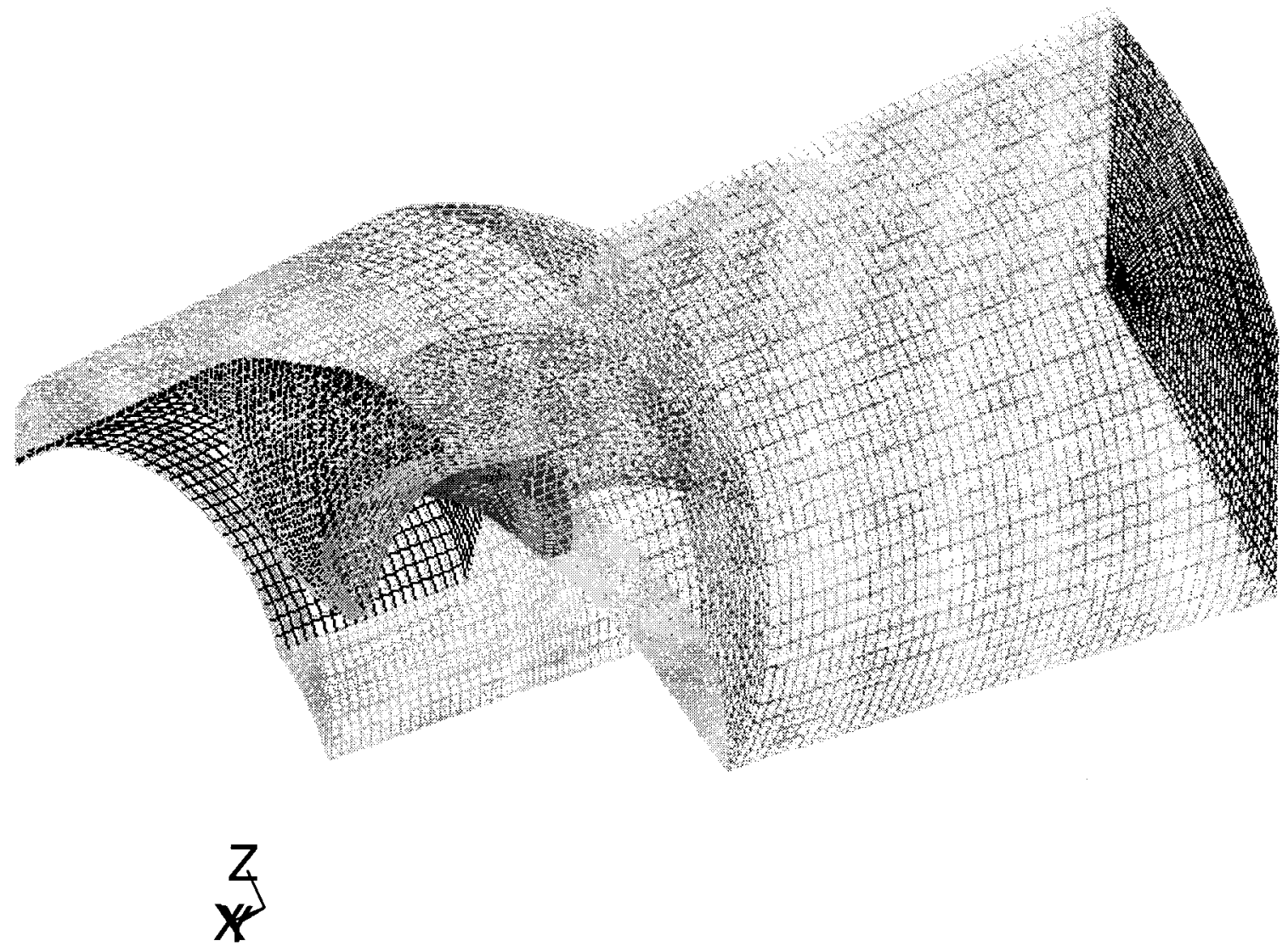

Figure 3. Meshes on three-dimensional domain 


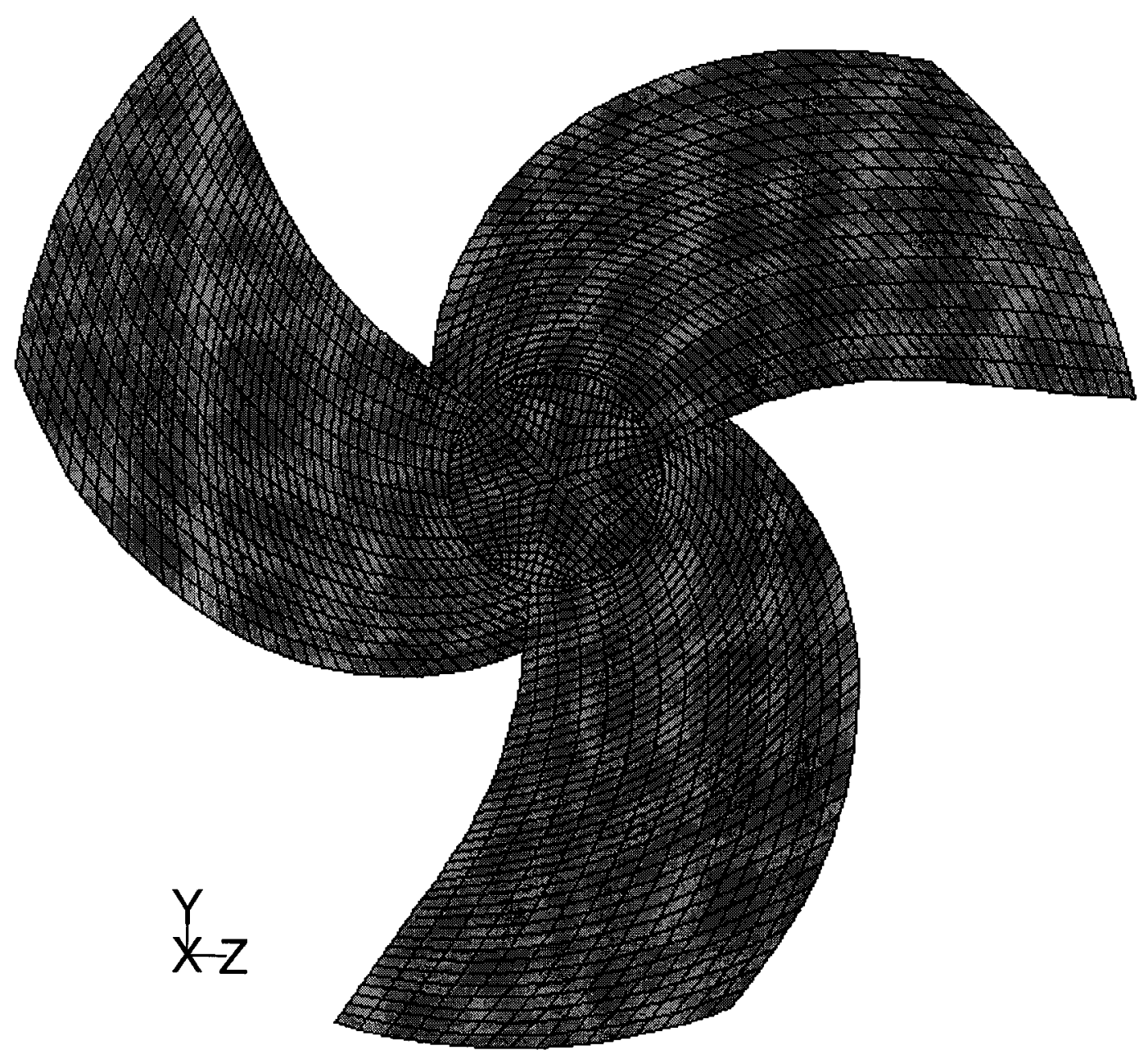

Figure 4. The blade and shaft surface grid after two repetitions of periodic boundary 


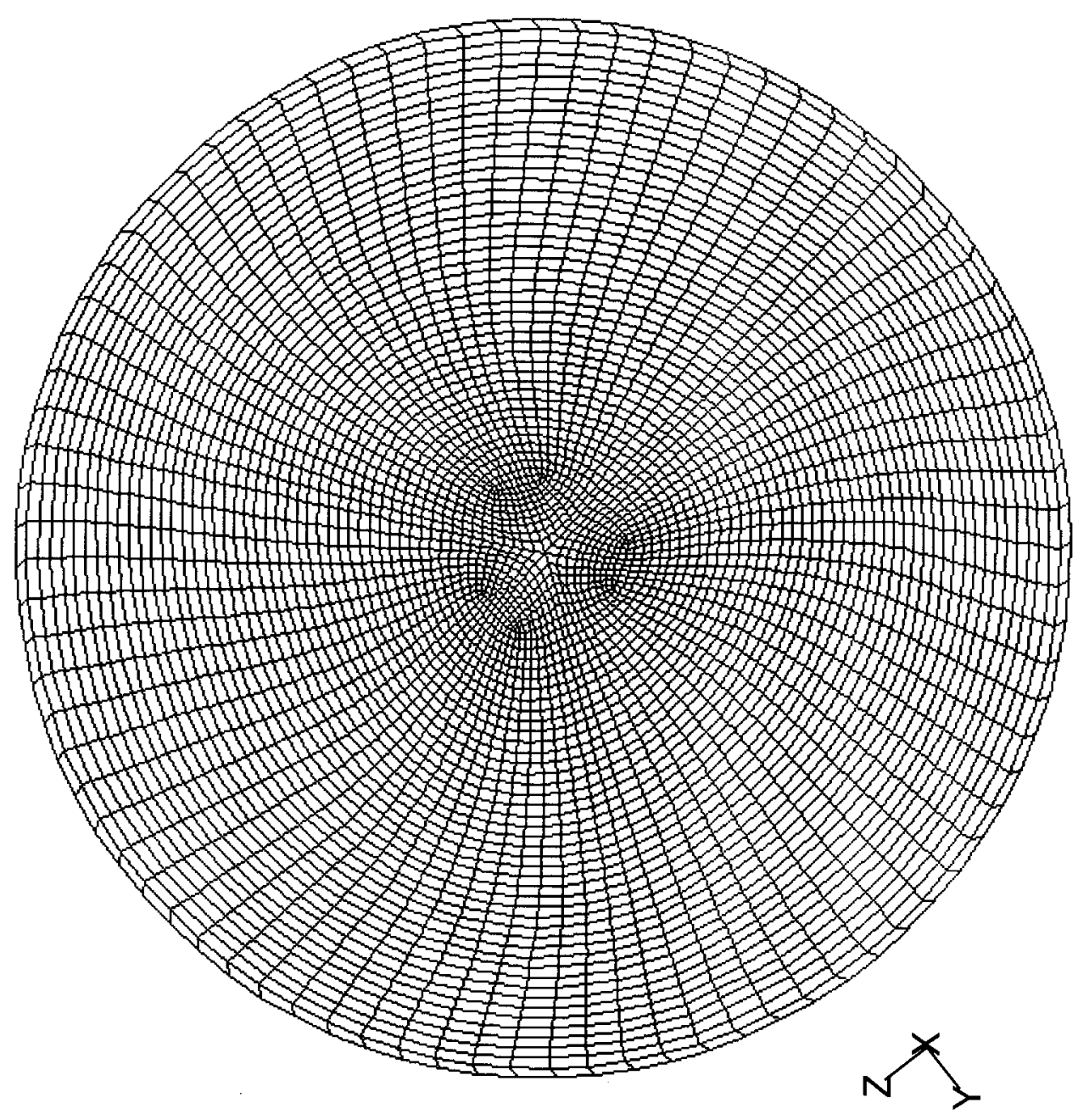

Figure 5. Two-dimensional meshes at exit plane after two periodic repetitions of the present model 


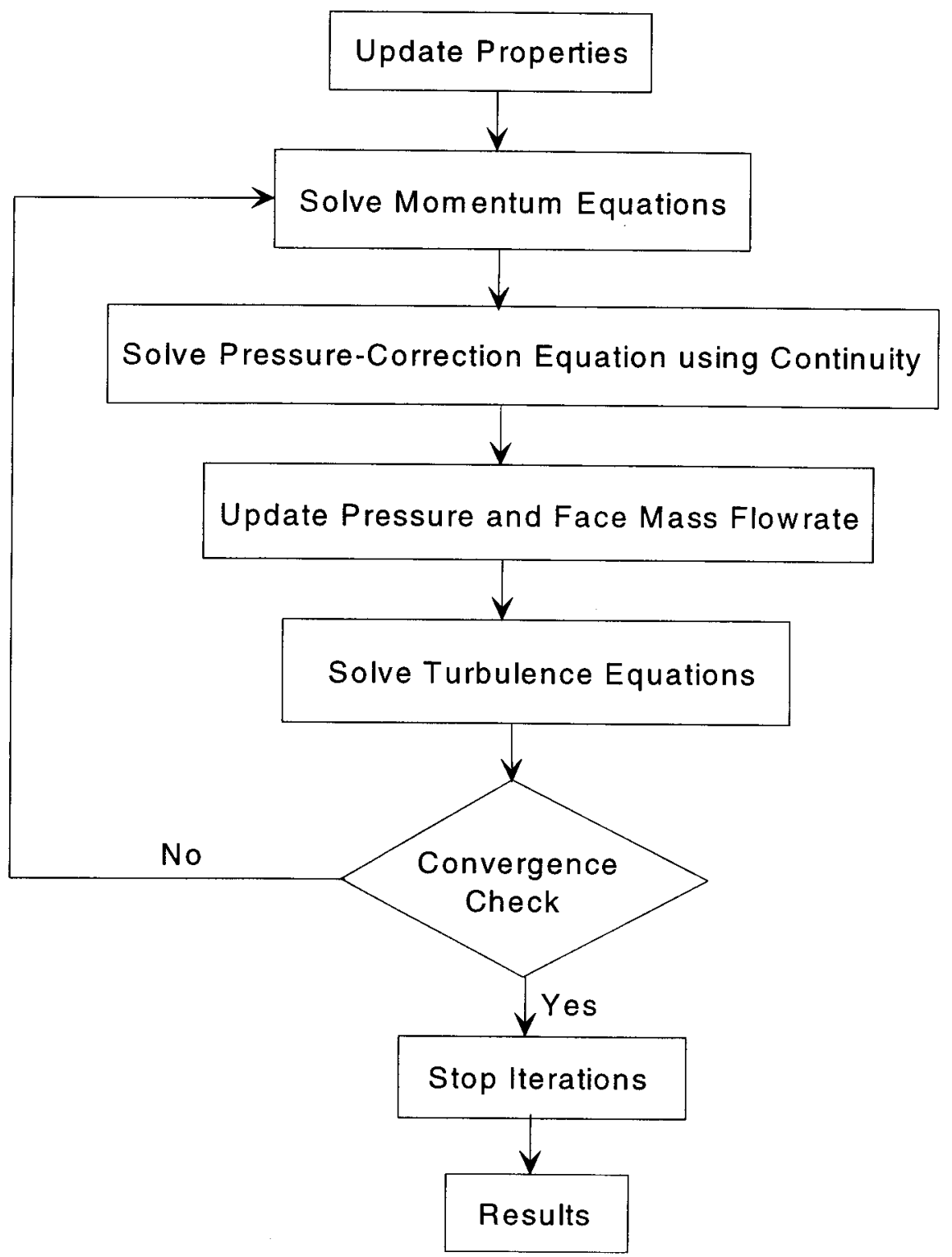

Figure 6. Overview of segregated solution method for the present model

\section{Investigation of the Nominal Pump Operating Conditions}

As discussed earlier, a detailed CFD model of mixing performance using a high speed mixing pump within a large stagnant liquid tank was used to study the behavior of a submerged axisymmetric mixing pump. The mixing pump is defined as a mixer which is not obstructed by any solid boundary in its vicinity. The mixer generates relatively large circulation flows and mixes with the surrounding fluid forming shear layers all around. This 
is different from the mixer pump within Tank 19, which sucks liquid near the tank bottom (about 6 inches above the tank bottom) and discharges it in a horizontal direction parallel to the tank bottom. In this situation, the interaction between the wall and the mixer changes the flow pattern due to presence of the no-slip boundary in the direction parallel to the bottom plane. Sensitivity calculations showed that nonuniformity in the inlet flow distribution had a negligible effect on the discharge flow pattern. Therefore, the inlet flow distribution is modeled as azimuthally symmetrical. This assumption is expected to be valid for highly turbulent and chaotic flow around the pump inlet.

Recently, HLW personnel performed an experimental investigation of structural pump stability and flow velocity measurements at the shroud exit under a $4 \mathrm{ft}$ liquid level. The velocity measurement was based on a pitot-tubes, and located 2 inch from the top and bottom of the shroud wall. In this experiment, three different pump speeds of 600,700 , and $860 \mathrm{rpm}$ were used. The asymmetry effect of the flow velocity at shroud exit was found to be negligible. The results also showed that the velocity at the center of the shroud was much smaller than the velocity adjacent to the shroud wall.

The present CFD model estimated nominal operating flow conditions using the HLW test data measured at the exit of shroud. The CFD results are compared with the test data for a range of pump flowrates $(9,000$ to $12,000 \mathrm{gpm})$ as shown in Fig. 7 . Figure 8 also shows the comparative results for three different pump speeds with an assumption of $9,000 \mathrm{gpm}$ flowrate. From this comparison and the HLW test data, nominal operating flowrate of the FLYGT mixer was estimated to be about $9,000 \mathrm{gpm}$. This flowrate is about half of the original estimated flowrate and raises a question about the driving force for the liquid mixing and material suspension for the tank floor.

The liquid flow velocity induced by the FLYGT mixer in Tank 19 is the driving force to effect suspension and mixing of particles or sludge components in the waste tank. The efficiency of this mixing process primarily depends on several key parameters that affect the liquid flow performance, such as axial flow velocity, flow direction of the mixer discharge, and the location of the mixer. Thus, the axial flow velocity at a $40 \mathrm{ft}$ distance from the shroud exit should be examined to see if $9,000 \mathrm{gpm}$ pump flow can deliver acceptable flow performance.

The present model does not include the fluid region outside the shroud of the mixer as shown in Fig. 2. From the previous two-dimensional wall jet model (Ref. 8), the maximum velocity at a $40 \mathrm{ft}$ distance from the shroud exit was estimated to be about $5.6 \mathrm{ft} / \mathrm{sec}$ for an inlet velocity boundary condition corresponding to $9,000 \mathrm{gpm}$ as shown in Table 2 . Detailed flow distribution at the plane $40 \mathrm{ft}$ away from the shroud exit is presented as a function of height above the tank bottom in Fig. 9. As shown in Fig. 10, it is noted that the axial flow is asymmetrically developed by the presence of the tank wall as fluid moves to the downstream region of the shroud. In Fig. 11, flow velocity vector plot in the neighborhood of the pump shroud is shown when the inlet velocity at the downstream region of the propeller is highly non-uniform. Detailed velocity contour plots above the tank bottom are shown in Fig. 12. It is found that the two-dimensional results are consistent with the three-dimensional model results in the predictions of flow patterns within the shroud. 
Report:

Date:

Page:
WESTINGHOUSE SAVANNAH RIVER COMPANY THREE-DIMENSIONAL CFD FLYGT MIXER MODEL AND RESULTS

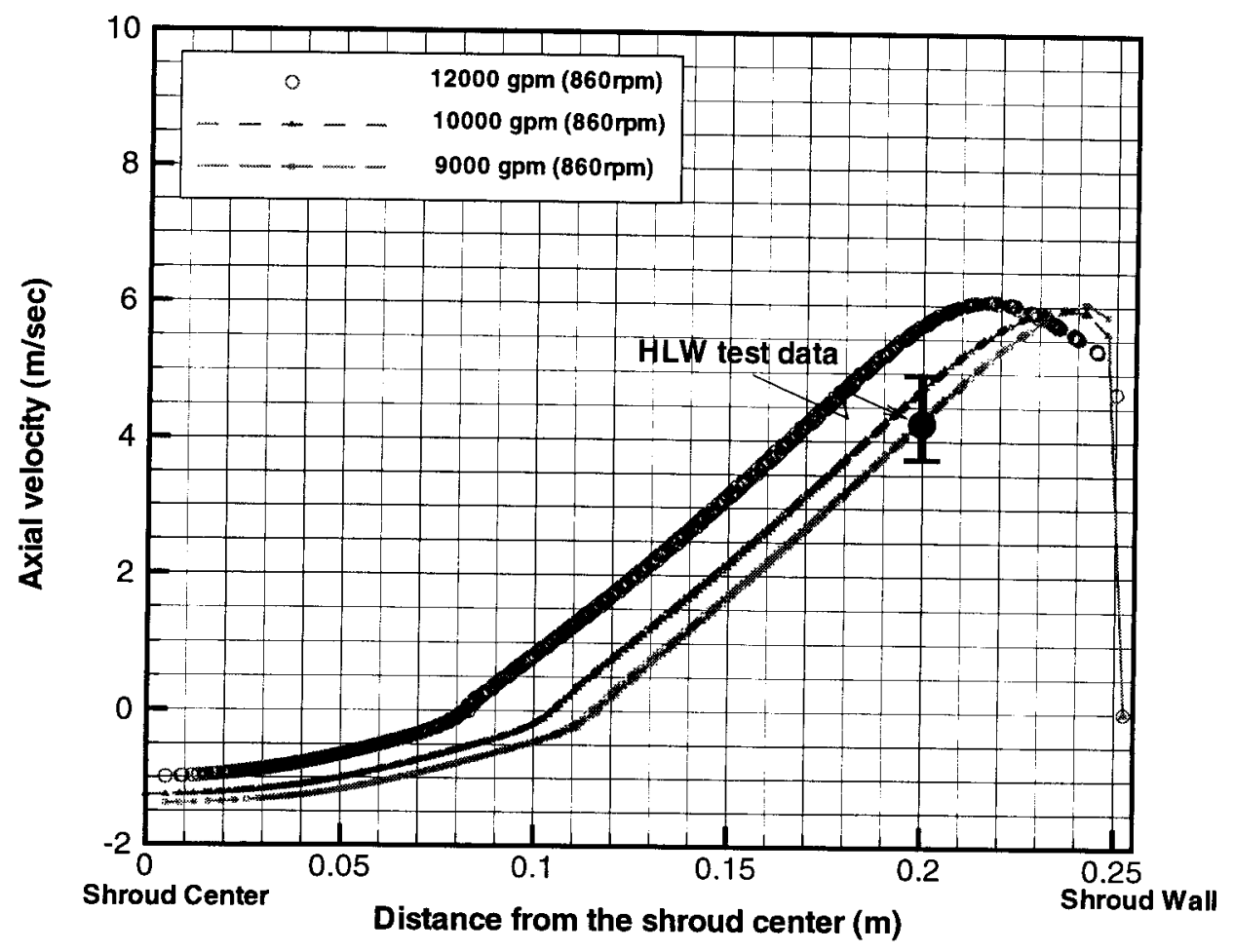

Figure 7. Comparison of predictions with test data 


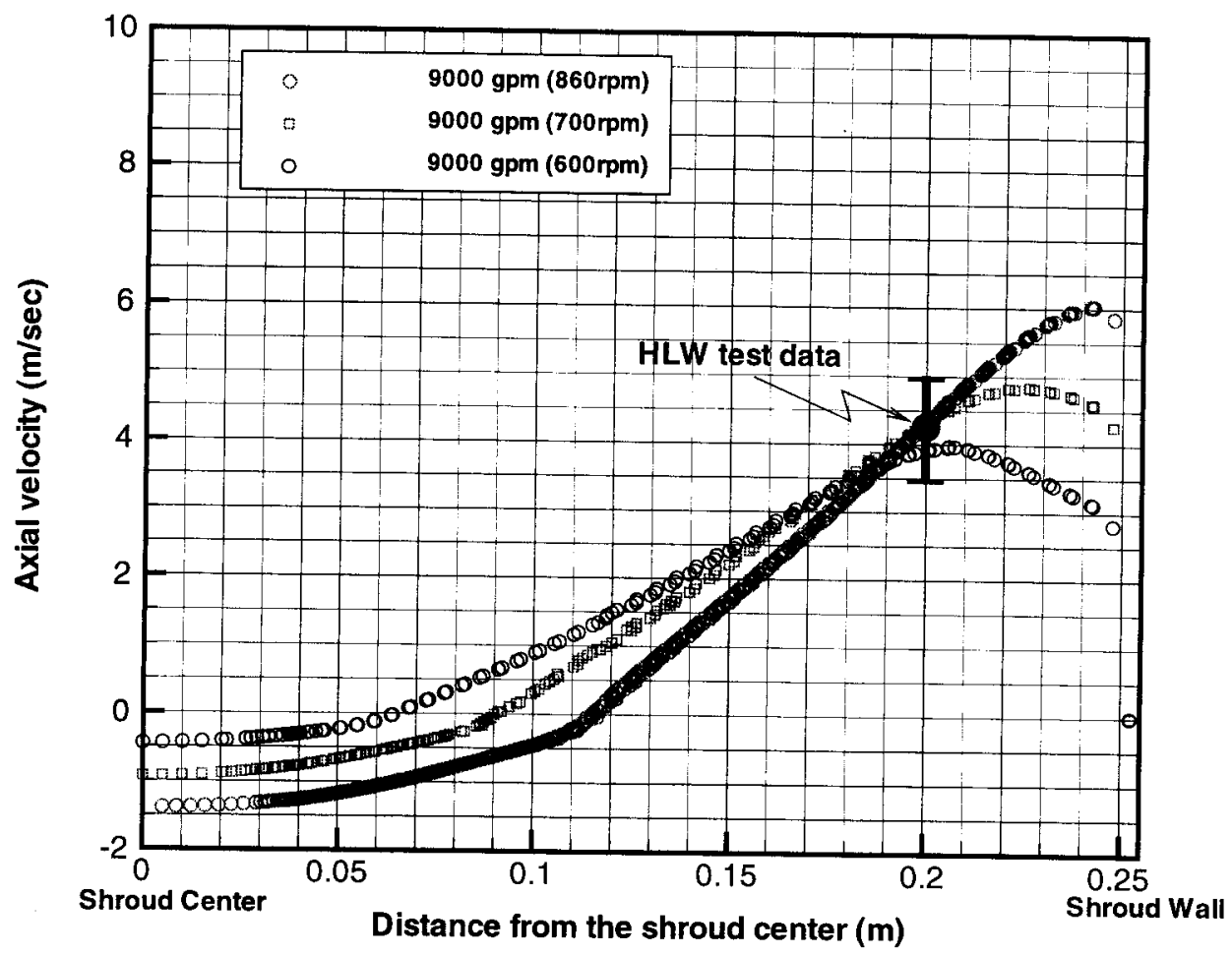

Figure 8. Determination of pump operating conditions 
Report:

Date:

Page:
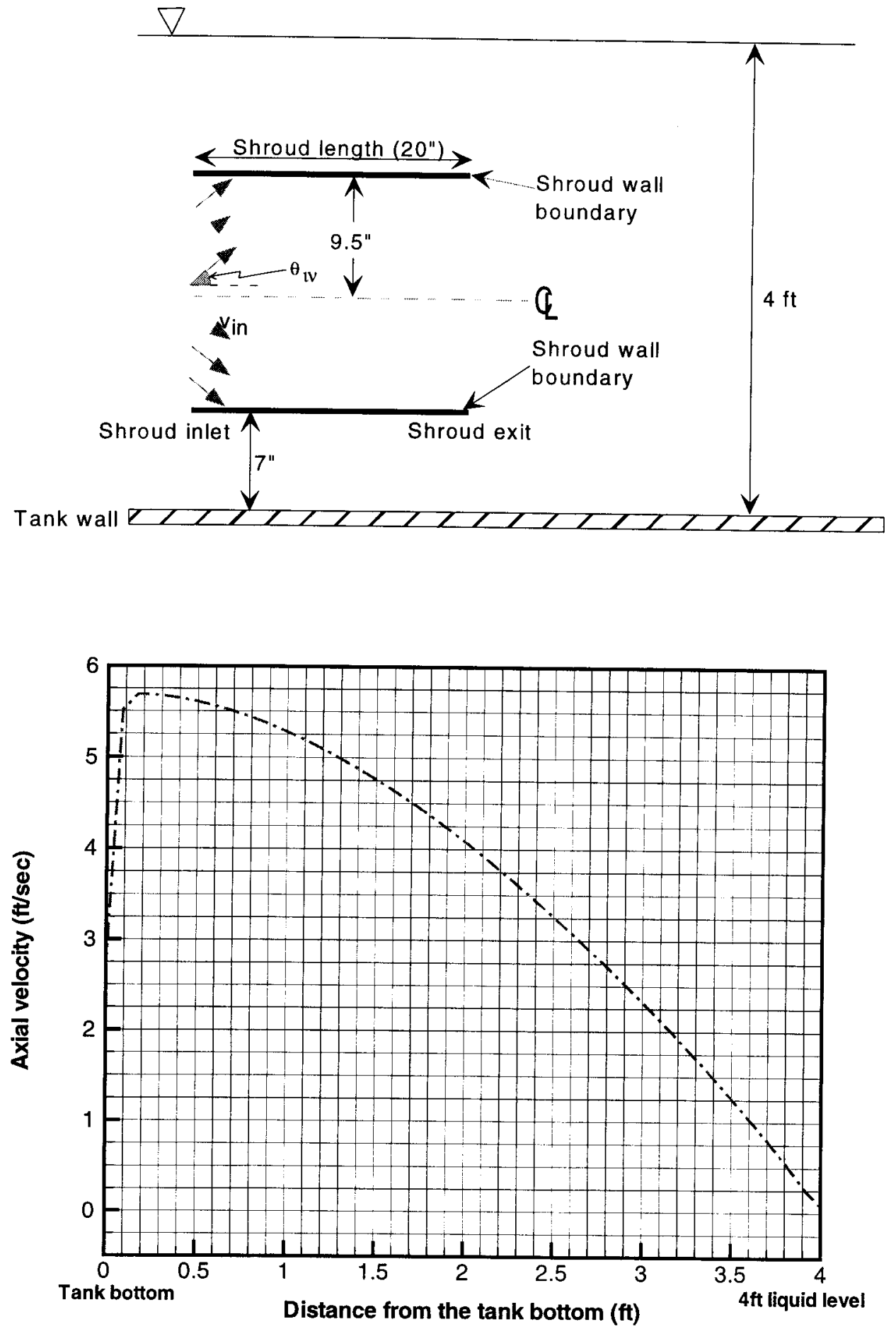

Figure 9. Axial velocity distribution as a function of liquid level at the 40 ft distance from the shroud exit based on two-dimensional wall jet model (Lee and Dimenna, 2000) for 9,000 gpm pump flowrate. Inlet velocity is uniformly $45^{\circ}$ skewed against the axial flow direction ( $\theta_{\text {in }}=45^{\circ}$ in the figure). 

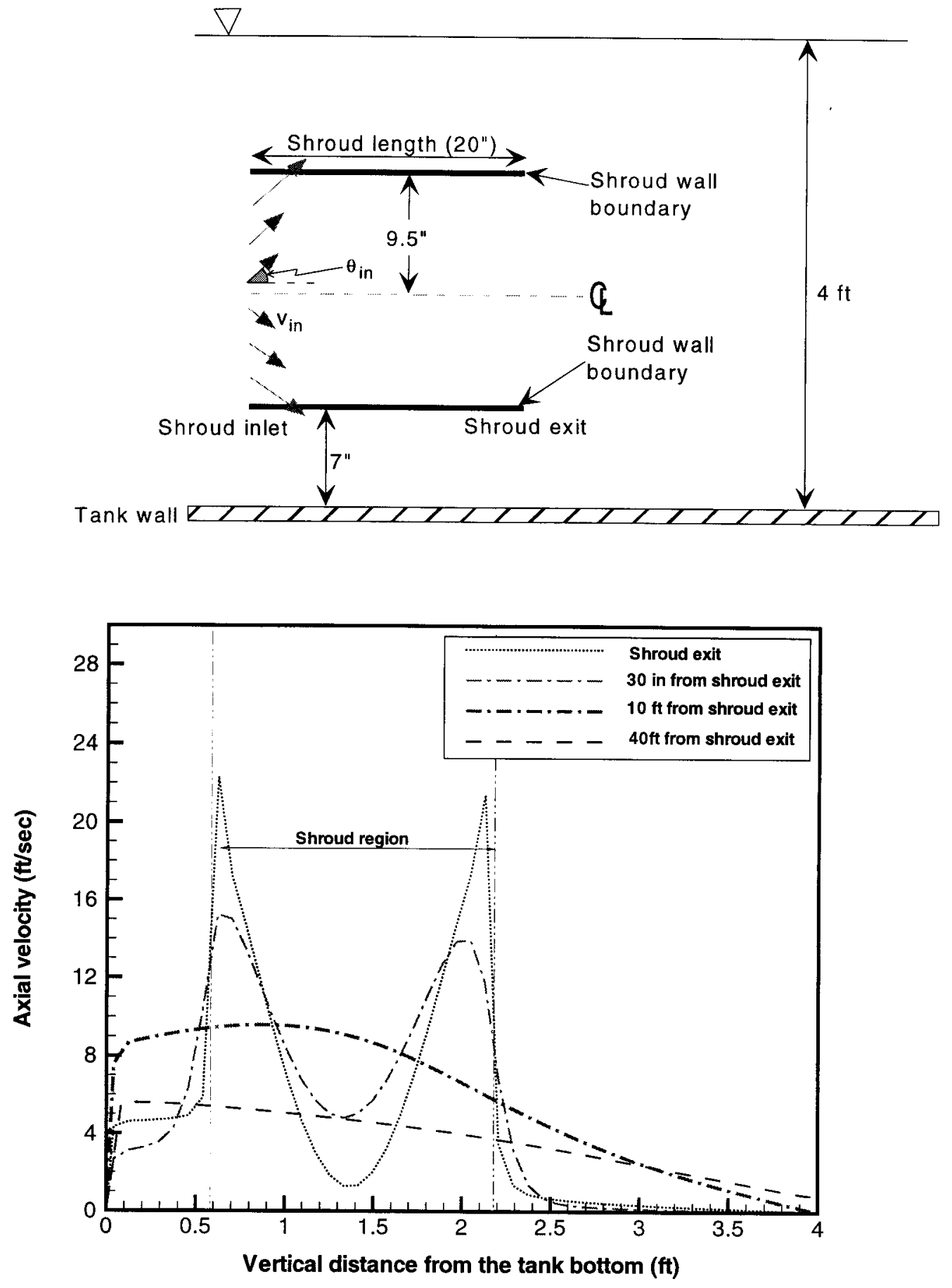

Figure 10. Axial velocity distributions as a function of liquid depth at various distances distance from the shroud exit based on two-dimensional wall jet model (Lee and Dimenna, 2000) for $9,000 \mathrm{gpm}$ pump flowrate. Inlet velocity is increased linearly along the radial direction. 

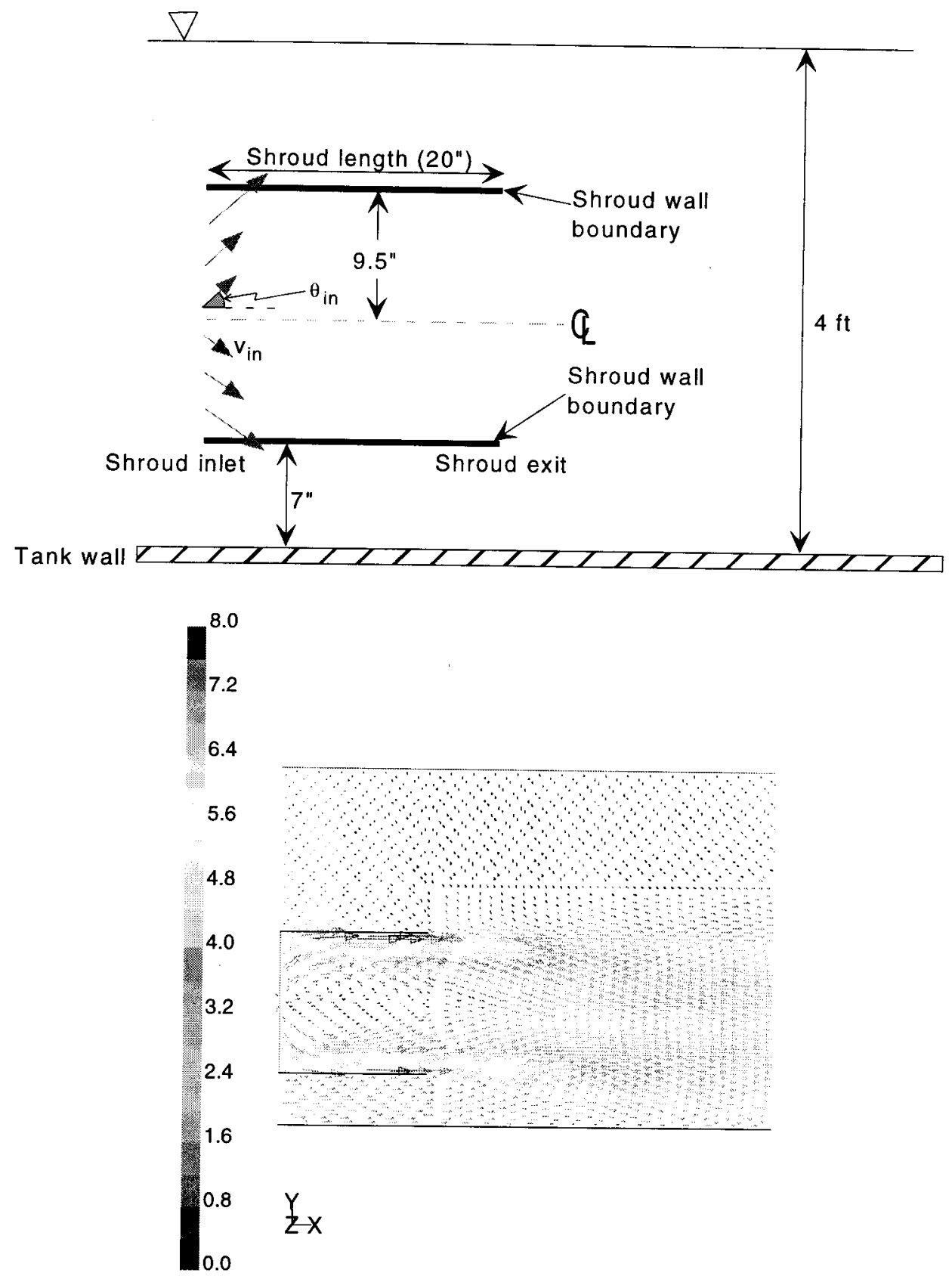

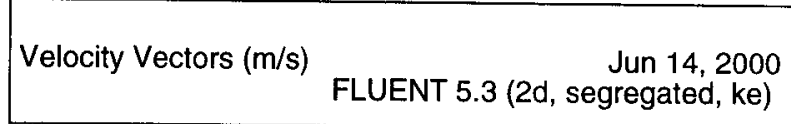

Figure 11. Velocity vector plot near the shroud region predicted by the two-dimensional wall jet model (Lee and Dimenna, 2000) for 9,000 gpm pump flowrate. Inlet velocity is increased linearly along the radial direction. 


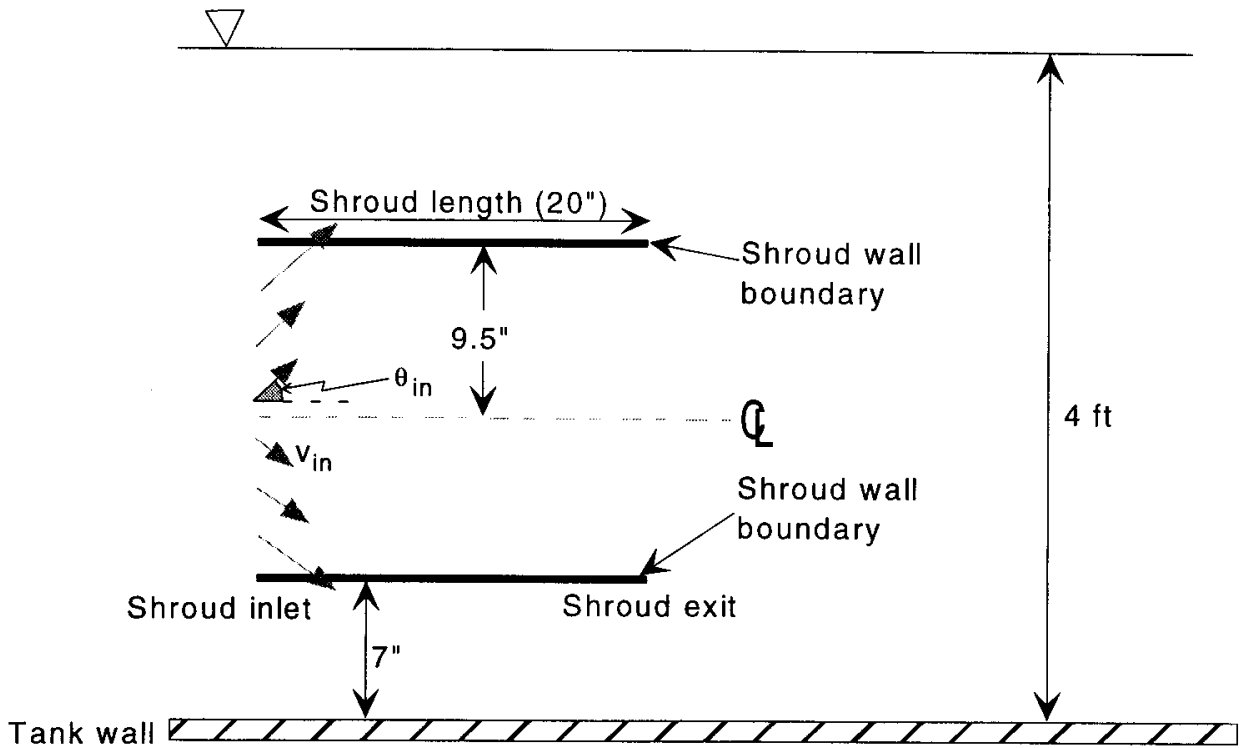

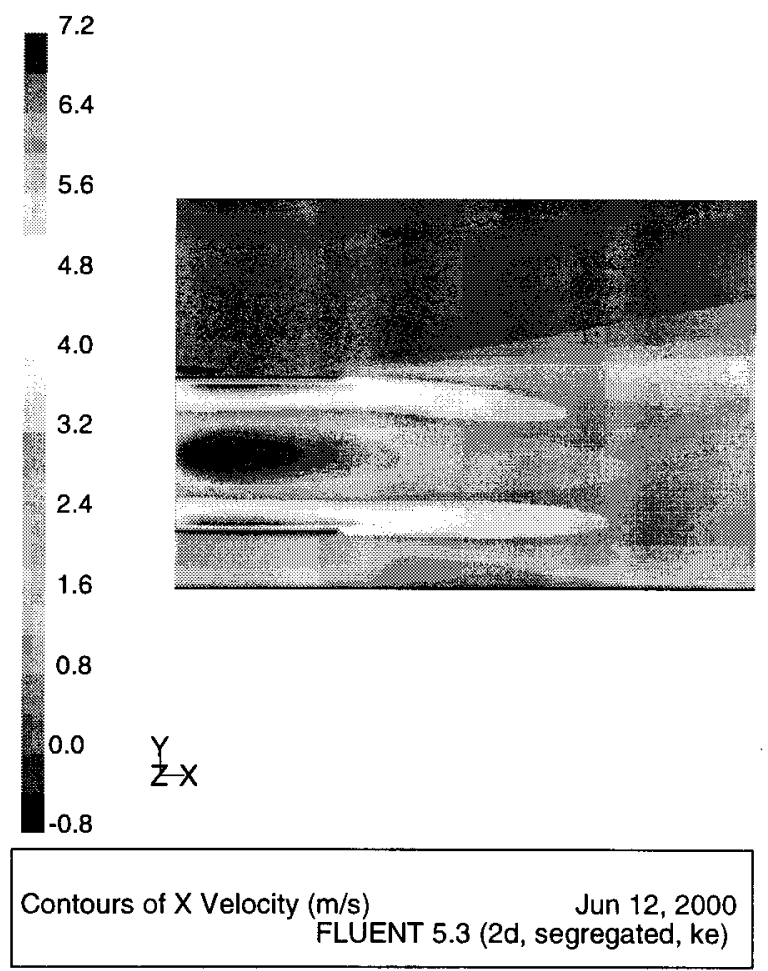

(Shroud region)

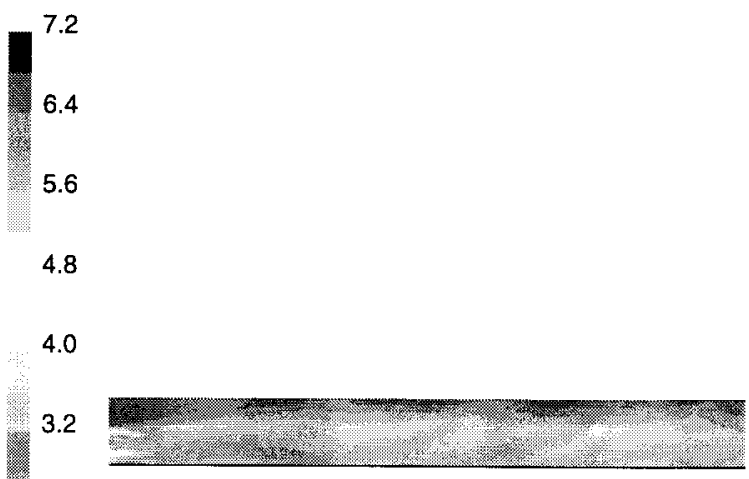

2.4

1.6

0.8

$0.0 \quad Y X$
$Z X$

$-0.8$

Contours of $X$ Velocity $(\mathrm{m} / \mathrm{s})$

FLUENT 5.3 (2d, segregated, ke)

(Whole domain)

Figure 12. Velocity contour plots near the shroud region and over the whole computational domain predicted by two-dimensional wall jet model (Lee and Dimenna, 2000) for 9,000 gpm pump flowrate. Inlet velocity is increased linearly along the radial direction. 


\section{Results and Discussions}

The results characterizing the overall flow behavior seen in the present calculations using the detailed three-dimensional CFD model with a prototypic propeller shape show a reasonably good representation of turbulent flow behavior. The three-dimensional computational domain and boundary for the model is shown in Fig. 1. The standard k- $\varepsilon$ model was used to capture the turbulent flow behavior of the FLYGT mixer. The flow velocity at the inlet of the mixer was about $3.3 \mathrm{ft} / \mathrm{sec}$ corresponding to $9,000 \mathrm{gpm}$ flow as shown in Table 2 . The inlet velocity was computed by assuming uniform flow distribution over the inlet geometry. The motor housing represents a flow blockage in the inlet region. Rotational effects of the pump propeller and hub regions were modeled by using the moving reference frame. The Reynolds number $(R e)$ at the inlet is about $1.4 \times 10^{6}$ for liquid water at room temperature, which corresponds to the fully-turbulent flow regime. Pressures shown in all contour plots of this report should be considered as gauge pressure with respect to one atmospheric pressure unless there is any description about the pressure.

\subsection{Steady-State Results}

All steady-state calculations were performed for turbulent flow using 120-deg symmetry and an unstructured mesh under a rotating reference frame. The turbulence effect in the model was represented by a two-equation model, standard $\kappa-\varepsilon$ model. The calculations have been performed under steady-state conditions to evaluate the loads on the propeller, hub, and shroud regions of the mixer under the operating flow conditions of 9,000 to $19,000 \mathrm{gpm}$. In addition, the steady-state model has been used to examine how sensitive the pump flow performance or cavitation phenomenon associated with deterioration of the pump performance is to a change of operating parameters or conditions. In this model, the density change due to pump cavitation was not considered. The main operating parameters used in the present analysis are rotational speed of the propeller, pump flowrate, flow profile entering the pump propeller, and surface roughness of shroud wall.

As mentioned earlier, the nominal operating flow condition was estimated by the steadystate CFD model using the HLW data (Adkins, 2000) measured at the shroud exit. From the present analysis and the literature, it is noted that pump cavitation is closely related to the pump performance in terms of performance deterioration and structural stability. The results showed that most cavitation occurred near the leading-edge tip of the pump blade as shown in Fig. 13. As shown in the figure, the lowest pressure is in the upstream region of the propeller blade causing the cavitation, while the highest pressure is in the downstream region of the blade. Table 3 shows minimum and maximum pressures around the leading tip of the propeller blade. The pressure difference across the leadingedge tip of the blade was about 38 psi for 860 rpm rotational speed as shown in Table 3. This is consistent with the literature data on the blade tips of ships' propellers. In the literature, this type of cavitation is often referred to as tip cavitation. Thus, this cavitation is caused by vorticity shed into the flow field just downstream of the blade tip. The results in Table 3 shows that cavitation doesn't occur when pump speed is lower than $500 \mathrm{rpm}$.

Figure 14 shows typical flow pattern and vorticity distributions near the downstream and upstream regions of the leading tip of the propeller blade. Maximum radial velocity 
occurred near the tip of the blade. Table 4 shows maximum radial and axial velocities for various pump speeds at the leading-edge tip of the propeller blade for nominal operating flow condition $(9,000 \mathrm{gpm})$. In this situation, maximum flowrate was artificially kept constant for different pump speeds for the simulation of performance deterioration. When pump speed increased under the condition of flow constraint, radial velocity at the blade tip increased quickly, but maximum axial velocity was not changed as expected.

Vorticity is a measure of the instantaneous rotation rate of the fluid on the principal axes. The vorticity $(\varpi)$ is defined as

$\vec{\varpi}=\nabla \times \vec{v}$

The usefulness of vorticity in interpreting fluid flow patterns is that vorticity tracks only the effects of viscous force. This can be seen by noting that the viscous term in the momentum equation of incompressible flow can be written as

$$
\nabla \cdot \tau=\mu \nabla^{2} \vec{v}=-\mu \nabla x \vec{\varpi}
$$

Thus, unbalanced shear stress $(\tau)$ generates vorticity. Pressure forces and gravity forces in the momentum equation act through the center of mass of a particle and can not produce vorticity.

Under a steady-state flow condition, loads on pump blades, hub, and shroud have been estimated by the present CFD pump model for a wide rage of flowrates and pump speeds. All the results are shown in Table 5. The results showed that pressure force was dominant compared to viscous force. For a given flowrate, total load for each of the three regions increased rapidly as pump speed increased.

Table 3. Pressures near the leading-edge tip of the pump blade under 9,000 gpm water flowrate of steady-state operating condition (Water saturation pressure corresponding to room temperature is about -14.3 psig.).

\begin{tabular}{|c|c|c|c|c|}
\hline Pump speed & $500 \mathrm{rpm}$ & $600 \mathrm{rpm}$ & $700 \mathrm{rpm}$ & $860 \mathrm{rpm}$ \\
\hline Upstream pressure & $-10.2 \mathrm{psig}$ & $-13.4 \mathrm{psig}$ & $-18.6 \mathrm{psig}$ & $-28.6 \mathrm{psig}$ \\
\hline Downstream pressure & $4.6 \mathrm{psig}$ & $4.6 \mathrm{psig}$ & $4.7 \mathrm{psig}$ & $9.4 \mathrm{psig}$ \\
\hline
\end{tabular}

Table 4. Maximum axial and radial velocities for various pump speeds at the leading-edge tip of the pump blade under $9,000 \mathrm{gpm}$ flow constraint of steady-state condition.

\begin{tabular}{|c|c|c|c|c|}
\hline Pump speed & $500 \mathrm{rpm}$ & $600 \mathrm{rpm}$ & $700 \mathrm{rpm}$ & $860 \mathrm{rpm}$ \\
\hline Max. radial velocity & $5.6 \mathrm{~m} / \mathrm{sec}$ & $6.8 \mathrm{~m} / \mathrm{sec}$ & $8.1 \mathrm{~m} / \mathrm{sec}$ & $11.1 \mathrm{~m} / \mathrm{sec}$ \\
\hline Max. axial velocity & $10.5 \mathrm{~m} / \mathrm{sec}$ & $10.6 \mathrm{~m} / \mathrm{sec}$ & $10.8 \mathrm{~m} / \mathrm{sec}$ & $10.8 \mathrm{~m} / \mathrm{sec}$ \\
\hline
\end{tabular}


Changes in flowrate are harder to interpret because the flowrate was used a boundary condition for the calculation. As the flowrate was decreased for a given pump speed, the propeller became starved for flow. Cavitation increased and the pressure loads on the mixer components increased. All of this indicated a deterioration in pump performance with reduced flow.

As shown in Fig. 15, load for the propeller blade is the largest among the three regions. It is noted that load for each component is not sensitive to the pump flowrate. Figure 16 shows that radial velocity at the tip of the blade is very sensitive to the rotational speed of the mixer. When pump speed increased from 500 to $860 \mathrm{rpm}$, maximum radial speed increased from $5.6 \mathrm{~m} / \mathrm{sec}$ to $11.1 \mathrm{~m} / \mathrm{sec}$ as shown in Table 4. Static pressure distributions corresponding to the velocity distributions are quite different depending on rotational speed of the mixing pump as presented in Fig. 17. These pressure distributions induce the load for each component of the mixer. As expected from our intuition, a large and localized vortex motion is generated near the leading-edge tip of the propeller blade. This large vortex motion introduces the cavitation, referred to as vortex cavitation in the literature (Knapp et al., 1970). Vorticity contour plots for the plane crossing the tip of the blade are shown in Fig. 18. The pressure coefficient along the axial flow direction crossing the leading-edge tip of the blade, as shown in fig. 19, indicates that the location of the minimum pressure is very close to the tip of the blade. This implies that the vortex cavitation inception will occur near the tip of the blade. It is seen that the negative minimum pressure coefficient $\left(-C_{p, m i n}\right)$ increases as the pump speed increases and the corresponding advance coefficient decreases, i.e., the propeller loading is increased. Comparisons of the numerical results for a wide range of non-dimensional pump speeds are summarized in Table 6 . The advance coefficient $\mathrm{J}$ is a dimensionless number associated with the propeller design condition, which is the ratio of fluid average velocity $\left(u_{\text {avg }}\right)$ to the product of pump speed $(n)$ and propeller diameter $\left(D_{\text {prop }}\right)$, that is, $J=u_{\text {avd }}(n$ $D_{\text {prop }}$ ). These results are consistent with the literature data (Hsiao and Pauley, 1999). The pressure coefficient $\left(C_{p}\right)$ is a dimensionless parameter defined by the reference pressure $\left(\mathrm{P}_{\mathrm{ref}}\right)$ and the reference dynamic pressure $\left(\mathrm{Q}_{\mathrm{ref}}\right)$. That is,

$$
C_{p}=\frac{\left(P-P_{\text {ref }}\right)}{Q_{\text {ref }}}
$$

In eq. (5), $Q_{\text {ref }}$ is defined in terms of the reference velocity $\left(V_{\text {ref }}\right)$.

$$
Q_{\text {ref }}=0.5 \rho_{f} V_{\text {ref }}^{2}
$$

In Table 6 and Fig. 19, one atmospheric pressure and $6 \mathrm{~m} / \mathrm{sec}$ flow conditions were used as the reference values. The results shown in Fig. 19 indicate that cavitation occurs near the leading-edge tip of the blade for the 700 and $860 \mathrm{rpm}$ pump speeds, but it does not occur for the $500 \mathrm{rpm}$ speed. The $600 \mathrm{rpm}$ pump speed is the critical value for the cavitation to occur. Thus, pump speed should be decreased from $860 \mathrm{rpm}$ to about 500 rpm, which is close to the original design speed 440 rpm of the FLYGT mixer, if cavitation needs to be avoided for the structural vibration or stability of the mixer. The cavitation line shown in the figure is based on the saturation pressure of vapor corresponding to the room temperature water, although real flow effects such as random turbulent fluctuation and water quality are known to influence cavitation inception. Figure 20 shows the 
distributions of the fluid vorticity, defined by eq. (8), along the axial direction crossing the tip of the blade. It is noted that there are two peaks. The first peak occurs near the tip trailing edge, and the second one is dominant near the leading-edge tip of the blade. These results are consistent with the literature observations (Knapp et al., 1970).

Turbulence intensity contour plots at the plane of the leading-edge tip are shown for three different rotational speeds of the FLYGT mixer in Fig. 21. The turbulence intensity is defined as the ratio of the root-mean-square of the velocity fluctuations, $u$ ', to the mean flow velocity, $u_{\text {avg. }}$. When pump speed increases from $600 \mathrm{rpm}$ to $860 \mathrm{rpm}$ under 9,000 gpm of nominal flow condition, the turbulence intensity changes from $34 \%$ up to $50 \%$ as shown in Fig. 21 (note that color scales change for each plot.).

As discussed earlier, the present model consists of three major regions as a modeling domain. They are propeller blade, hub (shaft region), and shroud regions. Under the steady-state model, phase change is not allowed even for the cavitating situation near the tip of the blade. However, the transient model allows phase change of liquid fluid when system pressure is below the vapor pressure corresponding to the fluid temperature. Detailed static pressure distributions at the downstream region of the blade are shown in Fig. 22. Highest pressure is at the downstream side of the leading-edge tip of the propeller blade, but lowest pressure is at the upstream side of the blade tip as discussed earlier. Figure 22 shows the viscous shear stress contour plot on the blade surface.

Typical flow pattern near the propeller region for 9,000 gpm nominal flow and $860 \mathrm{rpm}$ pump speed is presented in Fig. 24. Flow is entering the propeller zone in a convergent way and then reaches maximum velocity in the radial direction at the tip of the propeller blade. After leaving the propeller region the fluid flows in a divergent direction. Near the leading tip region of the propeller a large local vortex was generated, which is consistent with the experimental observation in the literature (Knapp et al., 1970). Vorticity contour plot near the blade of the propeller is shown in Fig. 25. Vorticity contour plot near the hub zone of the propeller is presented in Fig. 26. Highest vortex was produced near the blade connection region of the hub. Static pressure and shear stress distributions corresponding to the vorticity distribution of Fig. 26 are shown in Figs. 27 and 28.

Cavitation at the shroud wall was greatest near the upstream region of the propeller, while compression occurred most near the downstream region of the propeller. Graphical results for static pressure and shear stress distributions are shown in Figs. 29 and 30.

Velocity at the exit of the pump shroud is important in the assessment of the pump performance in terms of mixing capability. In the present work a wide range of pump flow and operating conditions were performed by using the three-dimensional pump model with the FLYGT mixer propeller model. Results of the radial velocity distributions at the shroud exit are shown in Fig. 31. The results showed that the location of the peak velocity moved to the peripheral region with decreasing pump flow. A backflow region due to flow reversal near the center of the shroud increased with decreasing flow. Figure 32 shows threedimensional steady-state velocity vector plots for $860 \mathrm{rpm}$ and 12,000 gpm flow condition at the inlet and exit regions of the pump shroud. It should be noted that velocity near the center of the shroud are much lower than the peripheral velocity at the plane of the shroud exit. This is consistent with the HLW test data (Adkins, 2000). 
Report: WSRC-TR-2000-00205

Date: $04 / 22 / 02$

Page:

Table 5. Axial forces along the flow direction for propeller, hub, and shroud regions under wide range of steady-state pump operating conditions.

\begin{tabular}{|c|c|c|c|c|c|c|c|}
\hline \multicolumn{2}{|c|}{$\begin{array}{l}\text { Pump operating } \\
\text { conditions }\end{array}$} & \multicolumn{2}{|c|}{ Propeller region } & \multicolumn{2}{|c|}{ Hub region } & \multicolumn{2}{|c|}{ Shroud wall region } \\
\hline $\begin{array}{l}\text { Pump } \\
\text { flowrate } \\
\text { (gpm) }\end{array}$ & $\begin{array}{l}\text { Pump } \\
\text { speed } \\
\text { (rpm) }\end{array}$ & $\begin{array}{l}\text { Pressure } \\
\text { (N) }\end{array}$ & $\begin{array}{l}\text { Viscous } \\
\text { (N) }\end{array}$ & $\begin{array}{l}\text { Pressure } \\
\text { (N) }\end{array}$ & $\begin{array}{l}\text { Viscous } \\
(\mathrm{N})\end{array}$ & $\begin{array}{c}\text { Pressure } \\
\text { (N) }\end{array}$ & $\begin{array}{l}\text { Viscous } \\
(\mathrm{N})\end{array}$ \\
\hline \multirow{4}{*}{9,000} & 500 & -2152 & 8.5 & 35 & -2.2 & -16 & -1.8 \\
\hline & 600 & -2896 & 10.3 & 58 & -1.5 & -19 & 8.1 \\
\hline & 700 & -4352 & 13.6 & 71 & -1.3 & -25 & 13.8 \\
\hline & 860 & -7317 & 21.3 & 92 & -0.3 & -35 & 26.4 \\
\hline \multirow{2}{*}{10,000} & 700 & -4310 & 13.6 & 72 & -1.9 & -25 & 12.5 \\
\hline & 860 & -6978 & 20.7 & 98 & -0.7 & -36 & 23.1 \\
\hline \multirow{3}{*}{12,000} & 680 & -4020 & 14.7 & 63 & -3.9 & -30 & -2.1 \\
\hline & 700 & -4290 & 15.3 & 69 & -3.9 & -30 & -0.9 \\
\hline & 860 & -6255 & 19.5 & 111 & -2.7 & -39 & 18.9 \\
\hline \multirow{2}{*}{13,000} & 700 & -4155 & 16.2 & 67 & -4.8 & -33 & -4.8 \\
\hline & 860 & -5941 & 20.0 & 118 & -3.0 & -39 & 15.1 \\
\hline \multirow{2}{*}{19,000} & 700 & -1614 & 23.1 & 189 & -8.1 & -39 & -3.6 \\
\hline & 860 & -5229 & 27.3 & 132 & -9.9 & -54 & -11.7 \\
\hline
\end{tabular}

(Note: Conversion factor 1 pound-force $=4.448 \mathrm{~N}$ )

Table 6. Comparison of minimum pressure coefficients $\left(C_{p, \min }\right)$ for the advance coefficients (J) corresponding to various rotational pump speeds.

\begin{tabular}{|c|c|c|c|c|}
\hline $\begin{array}{c}\text { Pump speed } \\
(\mathrm{rpm})\end{array}$ & $\begin{array}{c}\mathrm{J} \\
(--)\end{array}$ & $\begin{array}{c}\text { Reynolds number } \\
(---)\end{array}$ & $\begin{array}{c}-\mathrm{C}_{\mathrm{p}, \mathrm{min}} \\
(--)\end{array}$ & $\begin{array}{c}\text { Total loading on the blades } \\
\text { (pound-force) }\end{array}$ \\
\hline 860 & 0.40 & $1.4 \times 10^{6}$ & 10.4 & 1645 \\
\hline 700 & 0.49 & $1.4 \times 10^{6}$ & 7.2 & 978 \\
\hline 600 & 0.57 & $1.4 \times 10^{6}$ & 5.3 & 651 \\
\hline 500 & 0.68 & $1.4 \times 10^{6}$ & 3.9 & 484 \\
\hline 440 & 0.78 & $1.4 \times 10^{6}$ & 3.0 & 342 \\
\hline
\end{tabular}


$5.4 \mathrm{e}+04$

$3.3 e+04$

$1.2 e+04$

$-8.9 e+03$

$-3.0 e+04$

$-5.1 e+04$

Upstream side

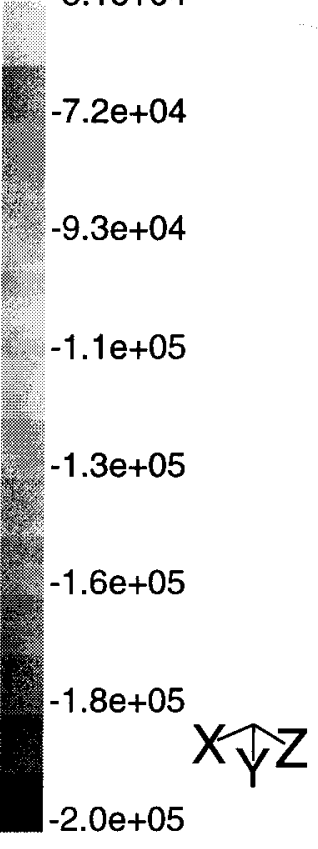

Figure 13. Typical static pressure distribution at downstream and upstream regions near the leading-edge tip of single pump blade for 860rpm of pump rotational speed and 9,000 gpm flow condition (Propeller blade shown in the figure rotates clockwise and the pressure unit is pascal in gauge.). 


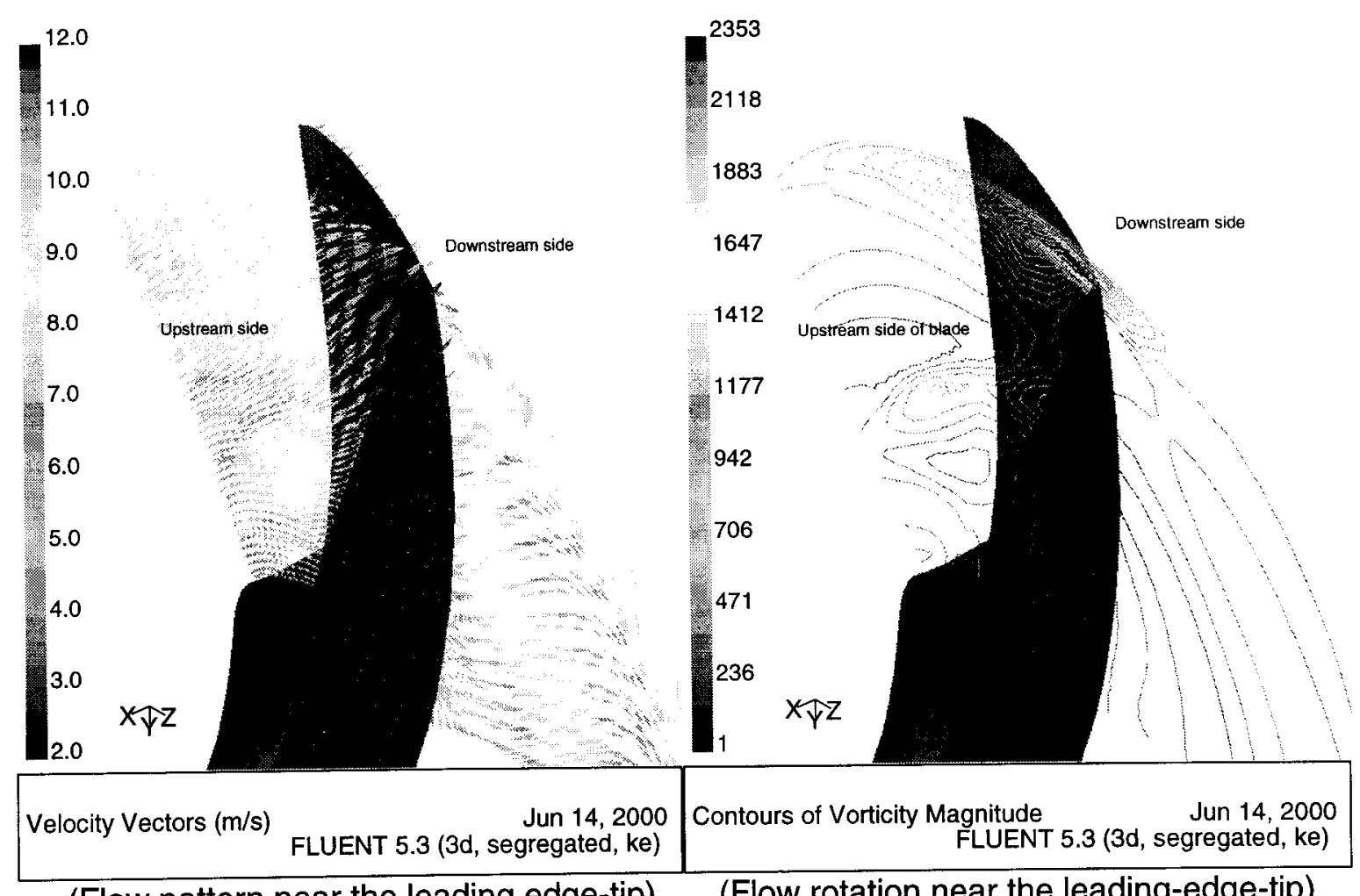

(Flow pattern near the leading edge-tip) (Flow rotation near the leading-edge-tip)

Figure 14. Typical fluid flow pattern and rotation profile at downstream and upstream regions near the leading-edge tip of single pump blade for $860 \mathrm{rpm}$ of pump rotational speed and 9,000 gpm flow condition (Propeller blade shown in the figure rotates clockwise.). 


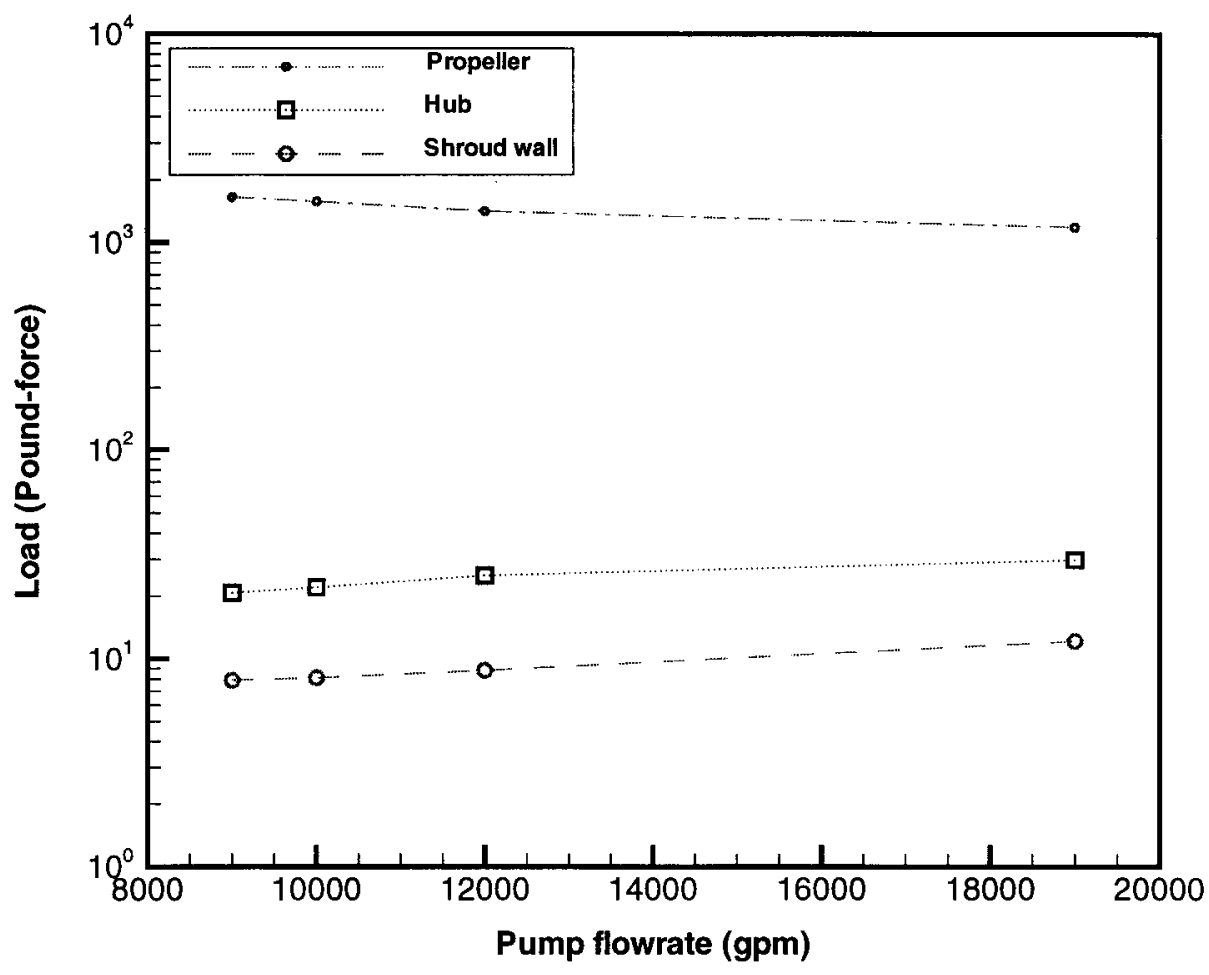

Figure 15. Load on each component of the FLYGT mixer along the axial flow direction for $860 \mathrm{rpm}$ of pump rotational speed (Loads for propeller and shroud wall region are negative forces). 

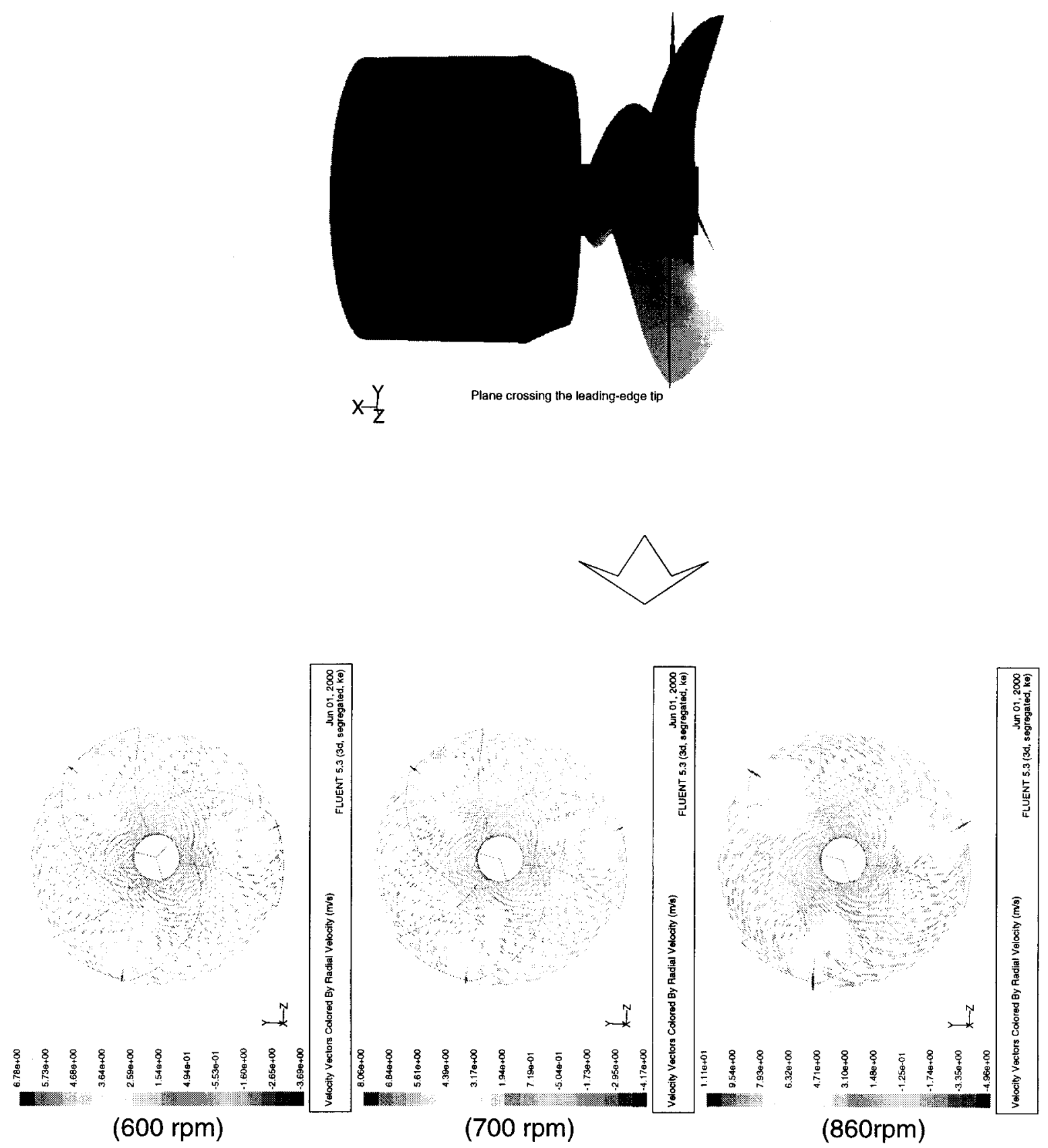

Figure 16. Radial velocity vector plots at the plane crossing the leading tip of the blade for various rotational speeds of $9,000 \mathrm{gpm}$ pump flowrate. 

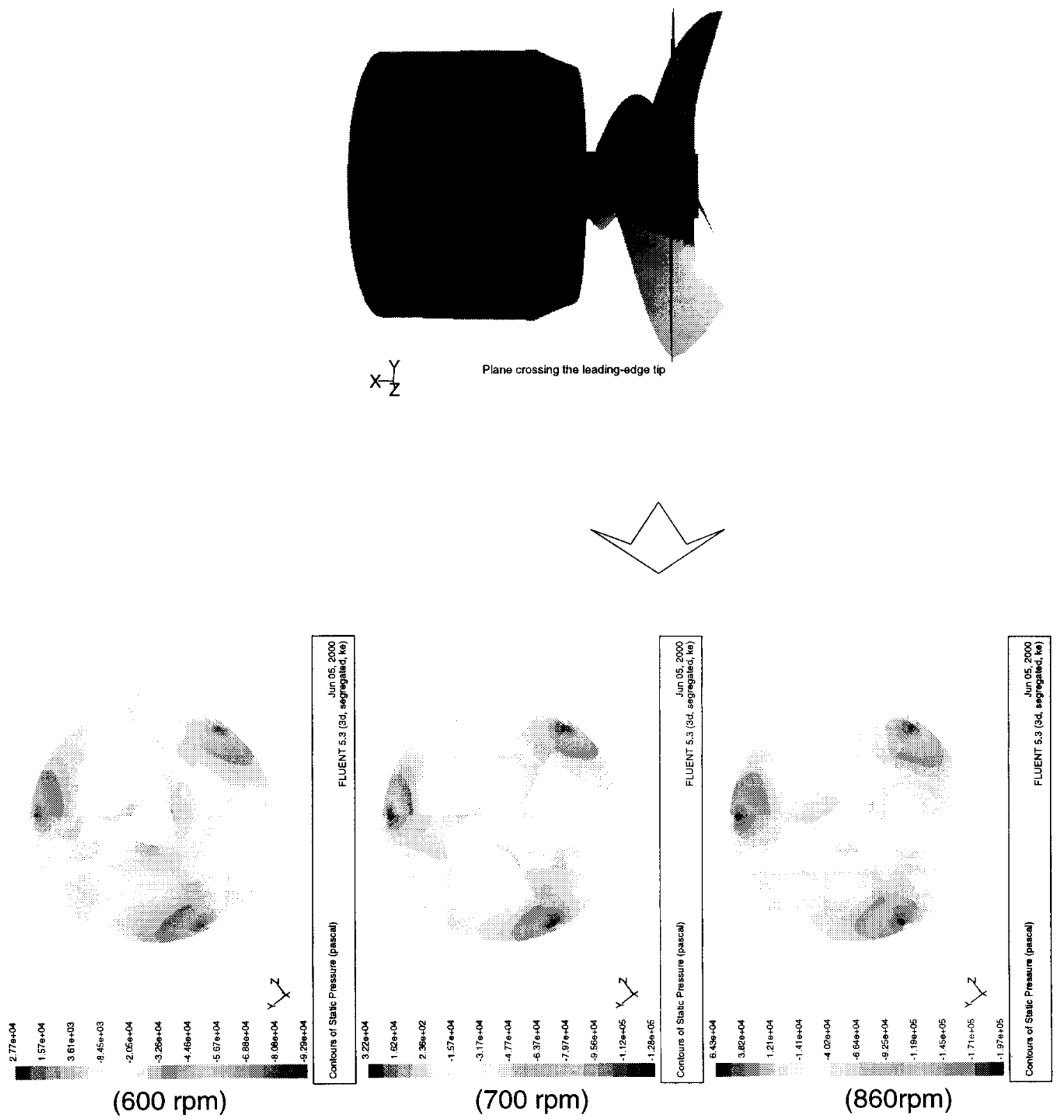

Figure 17. Static pressure contour plots at the plane crossing the leading tip of the blade for various rotational speeds of $9,000 \mathrm{gpm}$ pump flowrate. 

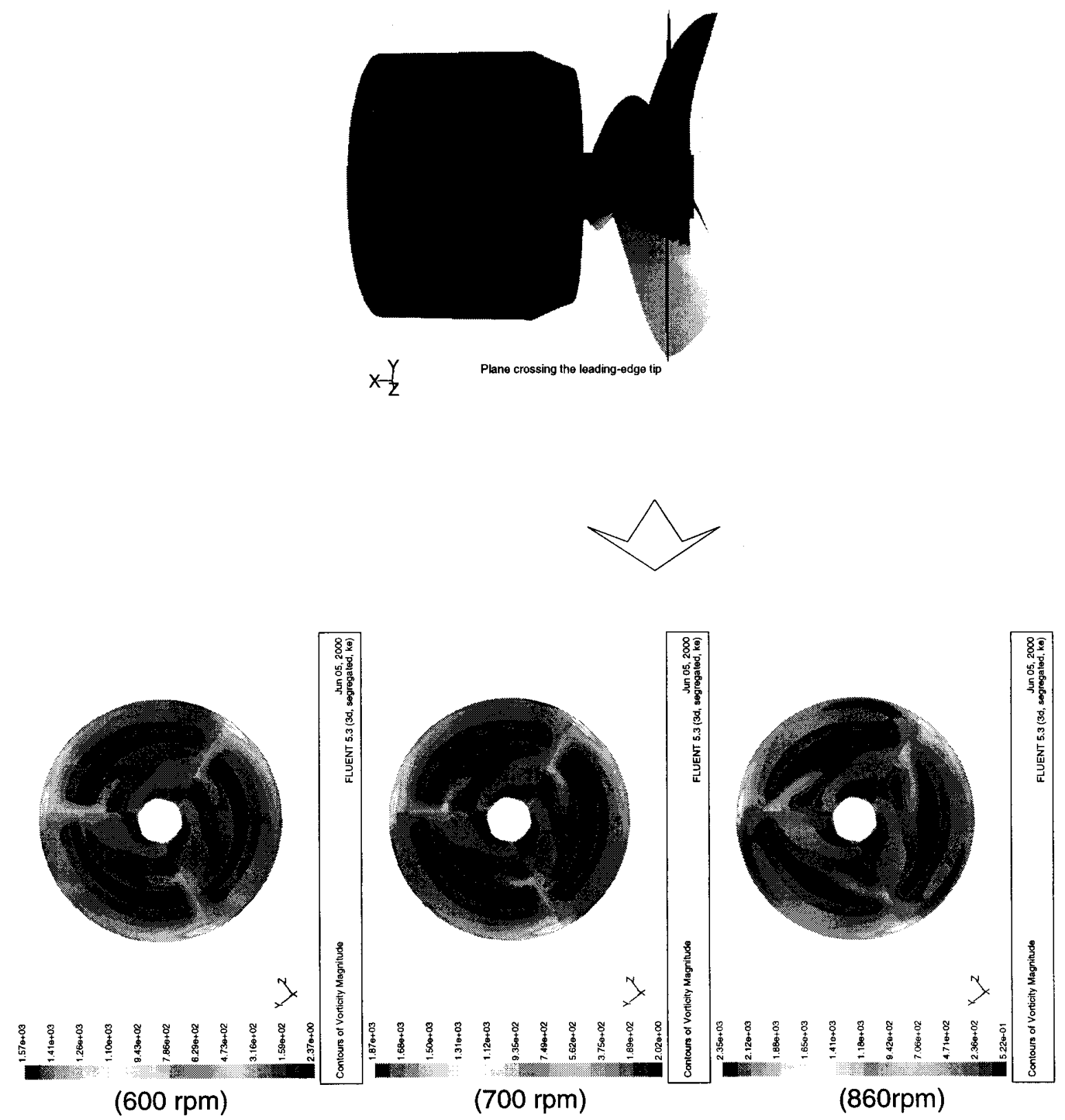

Figure 18. Vorticity contour plots at the plane crossing the leading tip of the blade for various rotational speeds of $9,000 \mathrm{gpm}$ pump flowrate. 

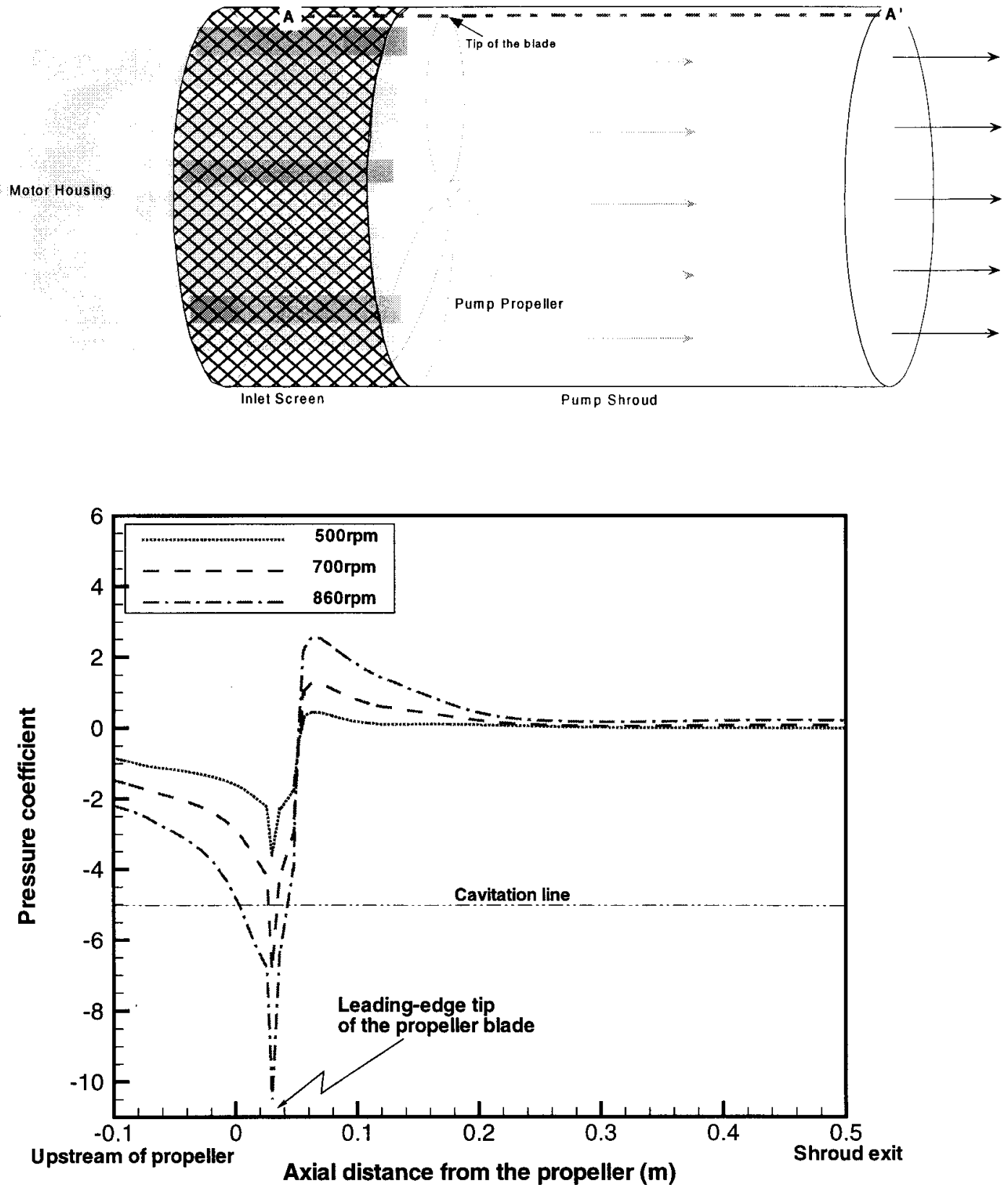

Figure 19. Pressure distributions along the axial flow direction (line A-A') crossing the leading-edge tip of the propeller blade for $9,000 \mathrm{gpm}$ flow condition as a function of pump speed. 
Report:
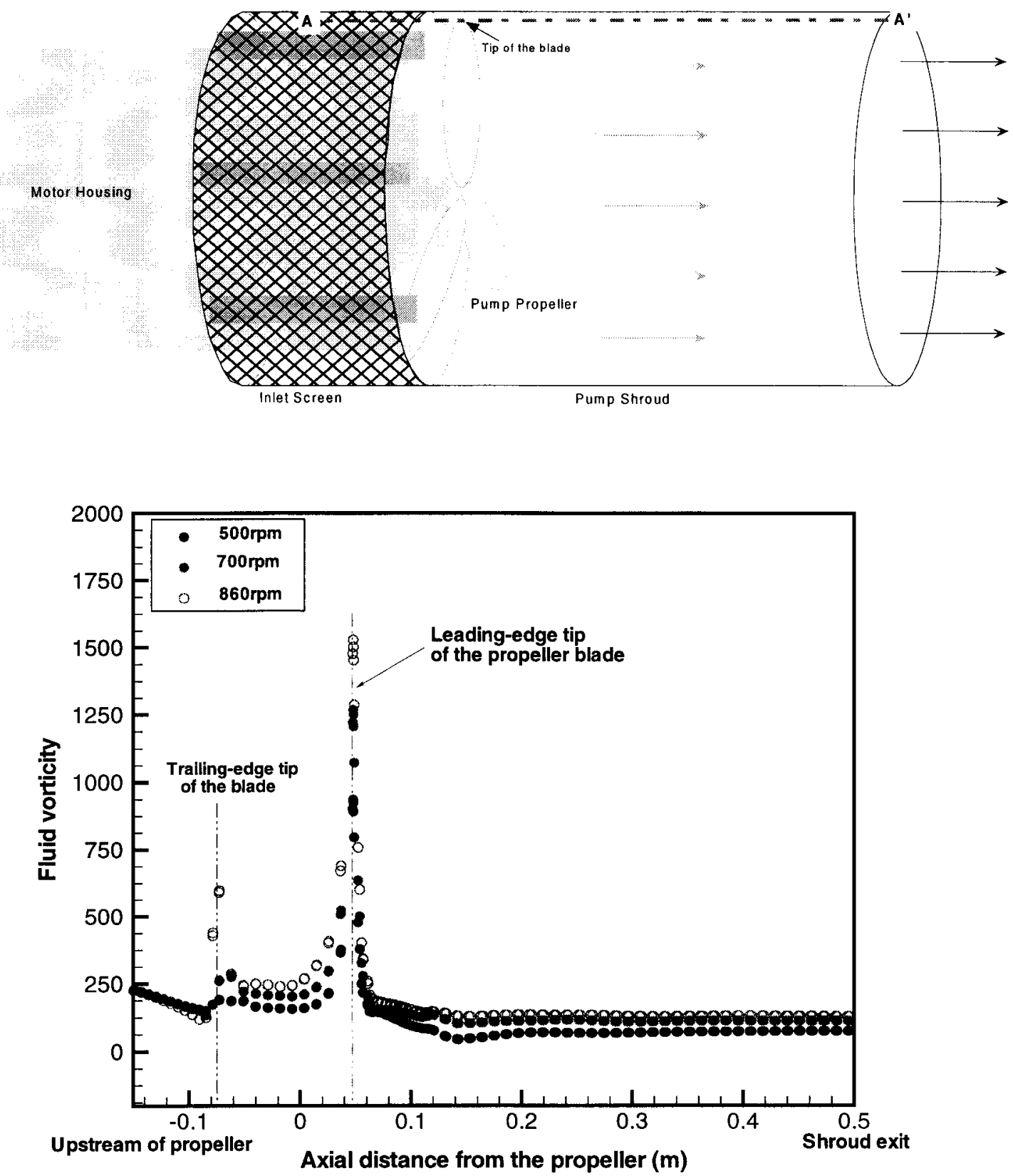

Figure 20. Fluid vorticity distributions along the axial flow direction (line A-A') crossing the leading-edge tip of the propeller blade for $9,000 \mathrm{gpm}$ flow condition as a function of pump speed. 

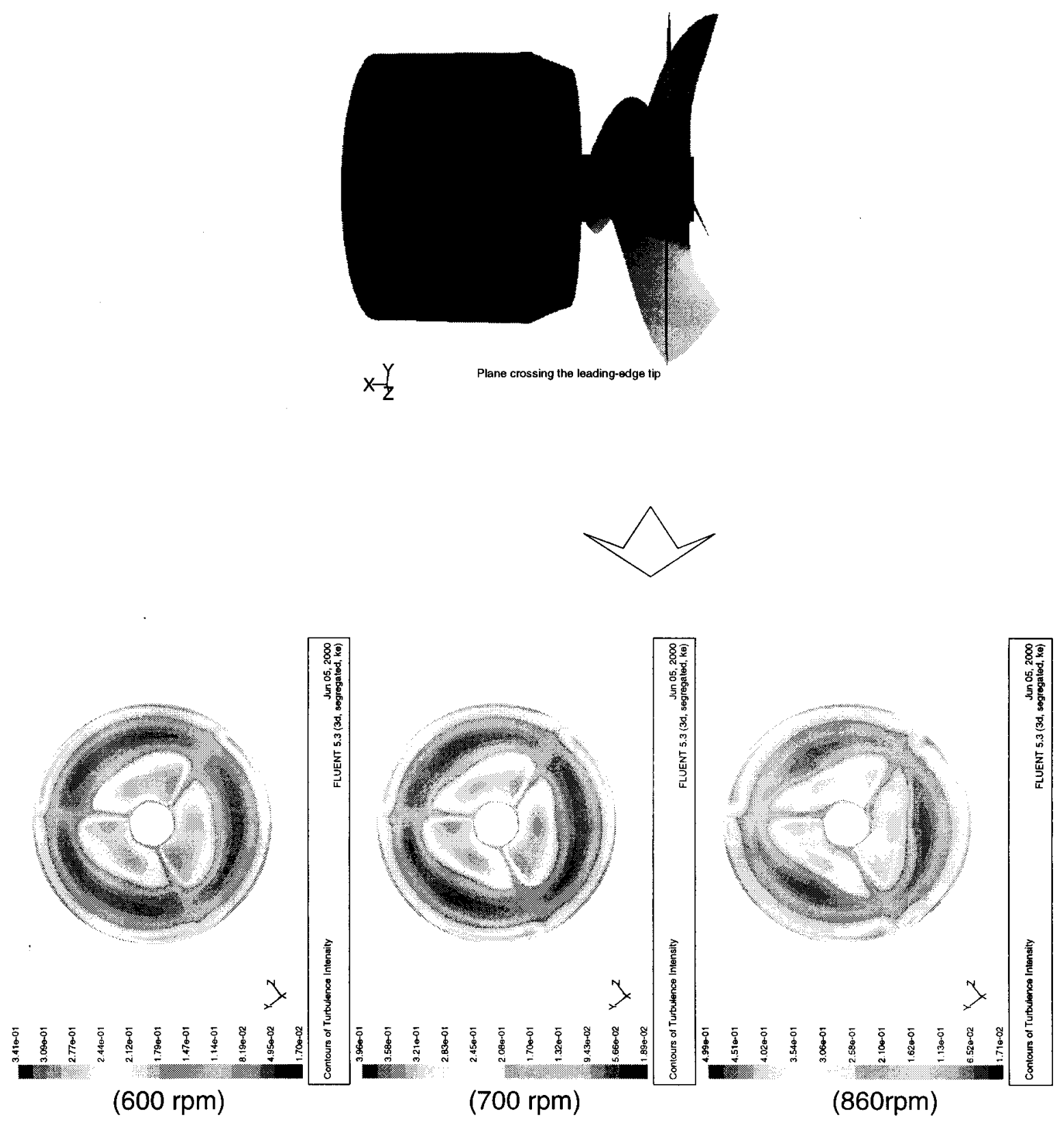

Figure 21. Turbulence intensity contour plots at the plane crossing the leading tip of the blade for various rotational speeds of $9,000 \mathrm{gpm}$ pump flowrate. 


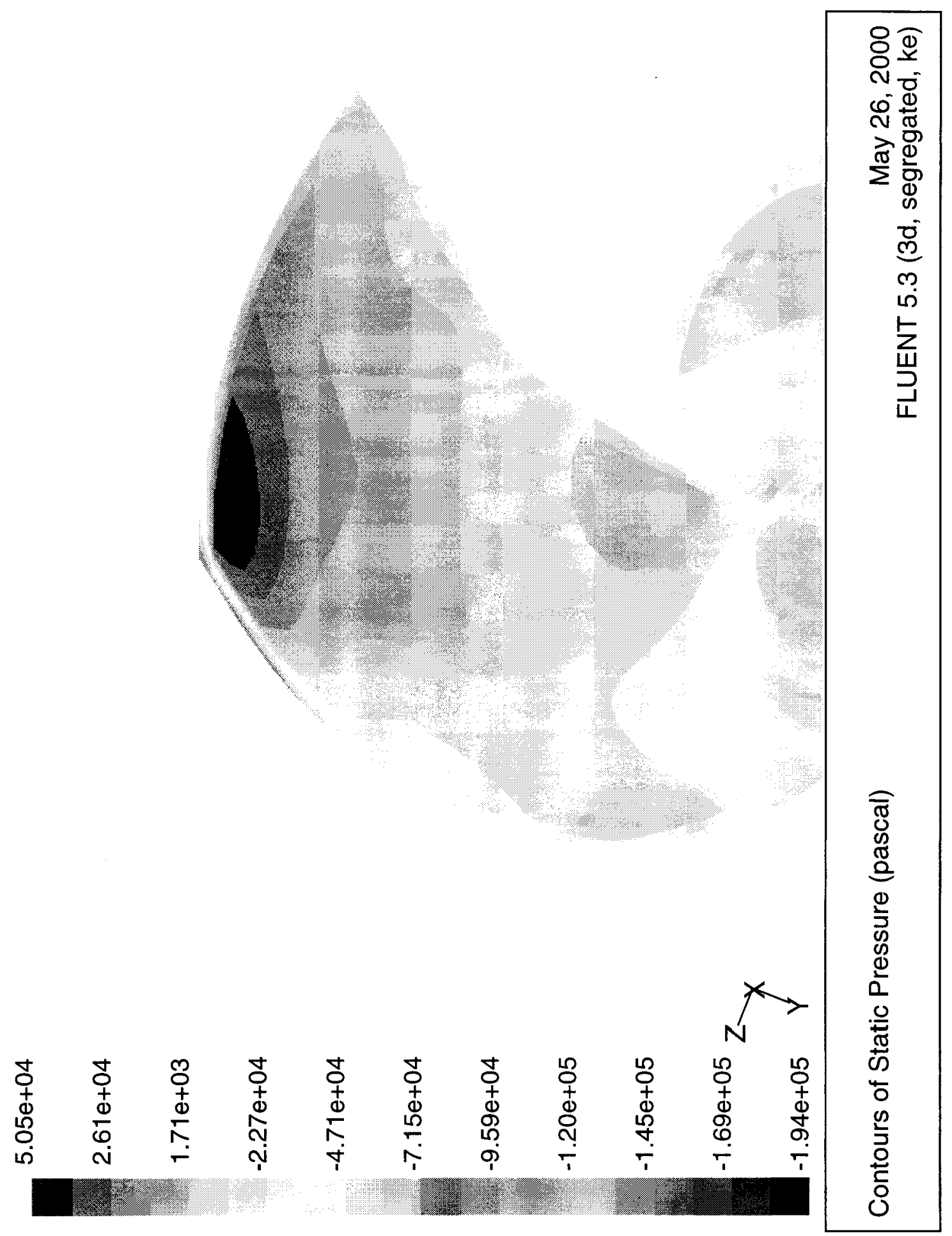

Figure 22. Static pressure contour plot on the propeller surface. 


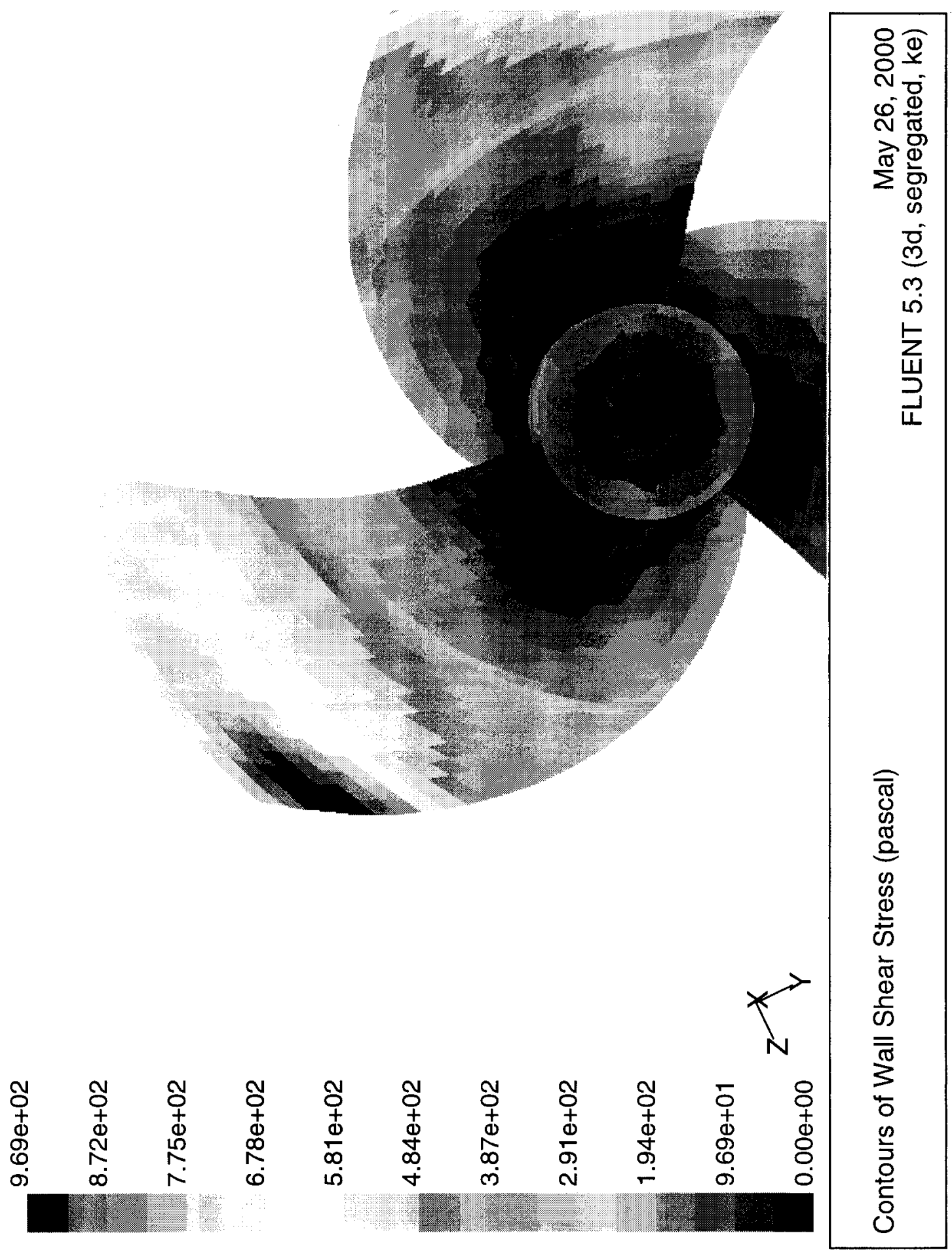

Figure 23. Shear stress contour plot on the propeller surface. 
Report:

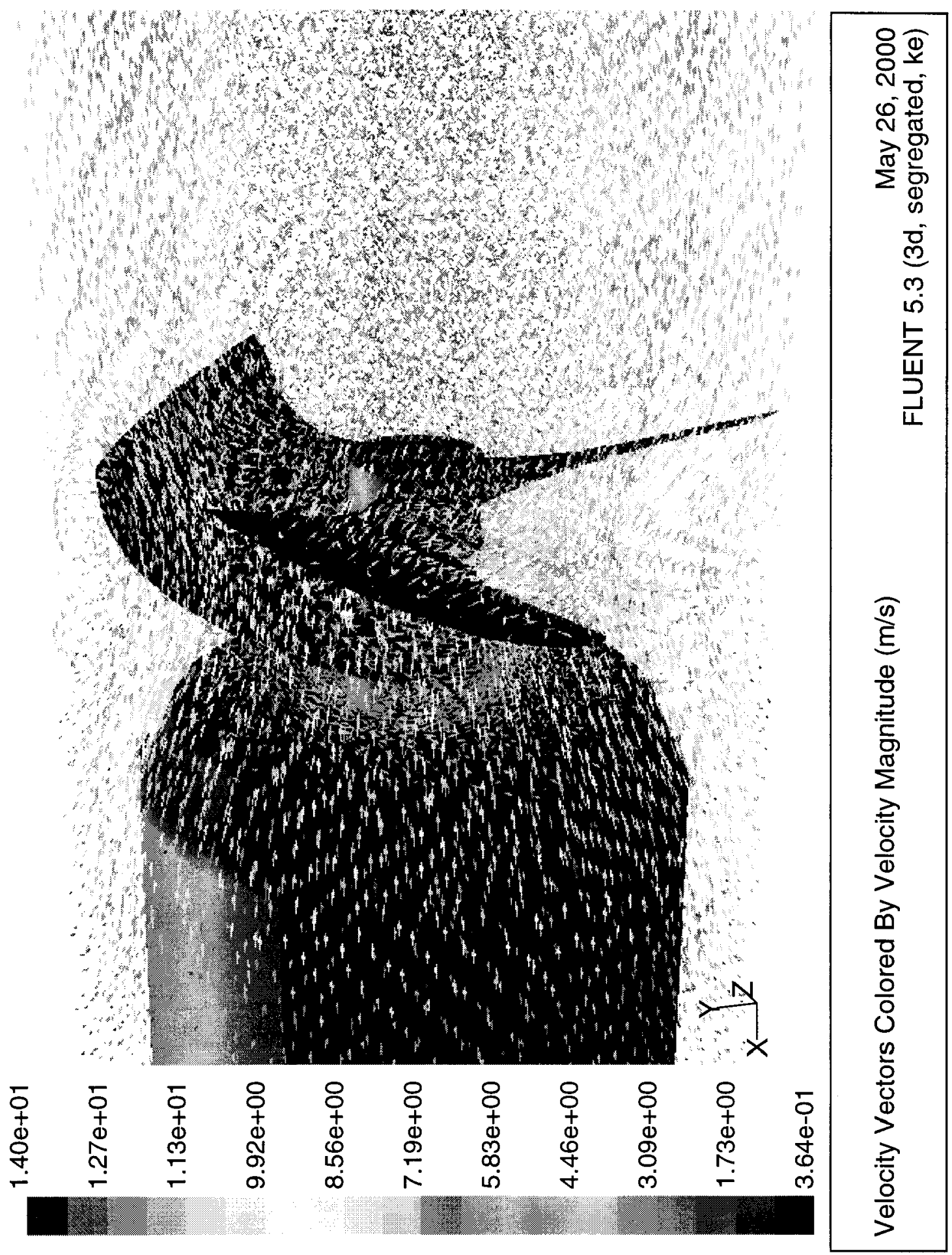

Figure 24. Velocity contour plot near the pump propeller. 


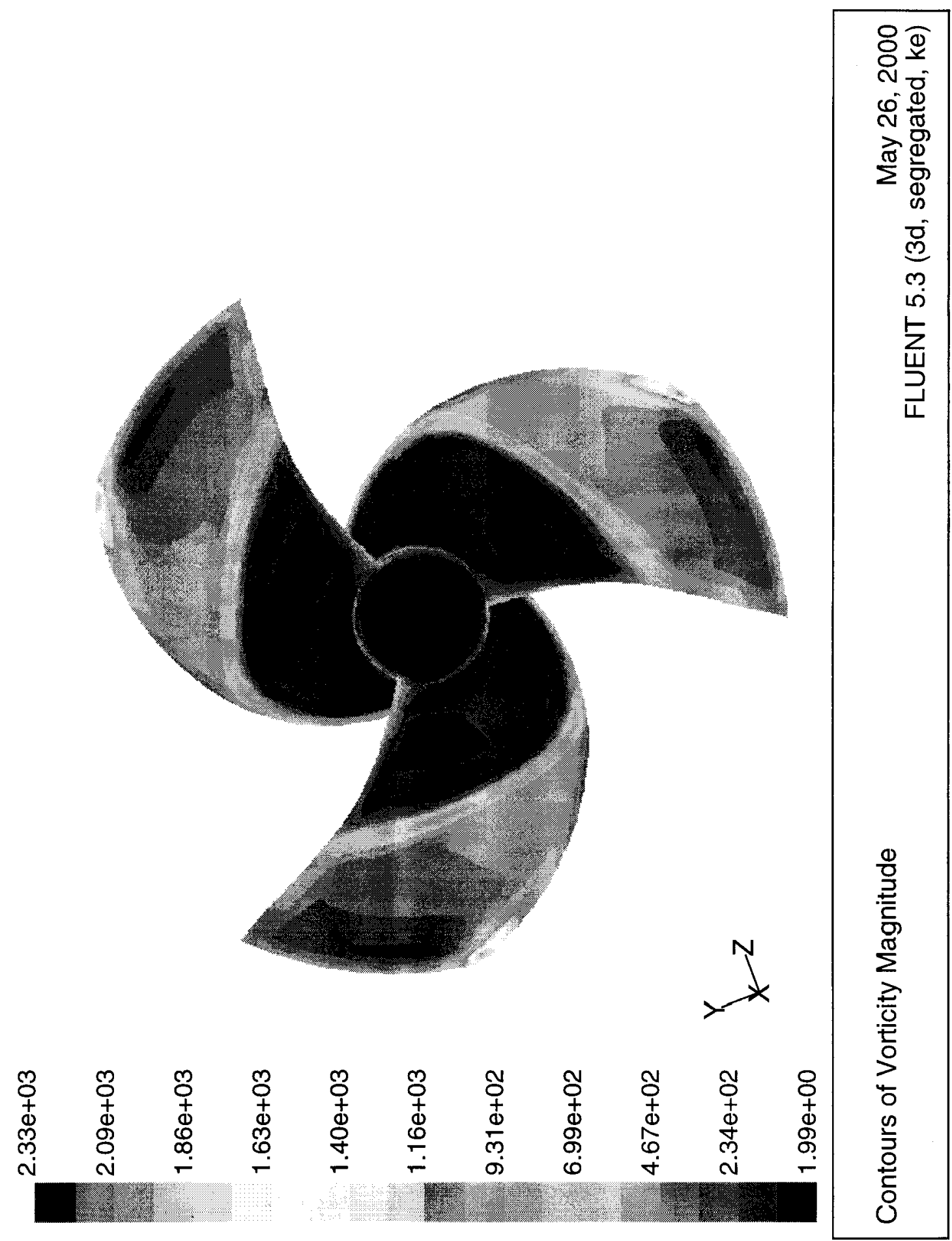

Figure 25. Vorticity contour plot at the pump propeller region (counterclockwise propeller direction). 


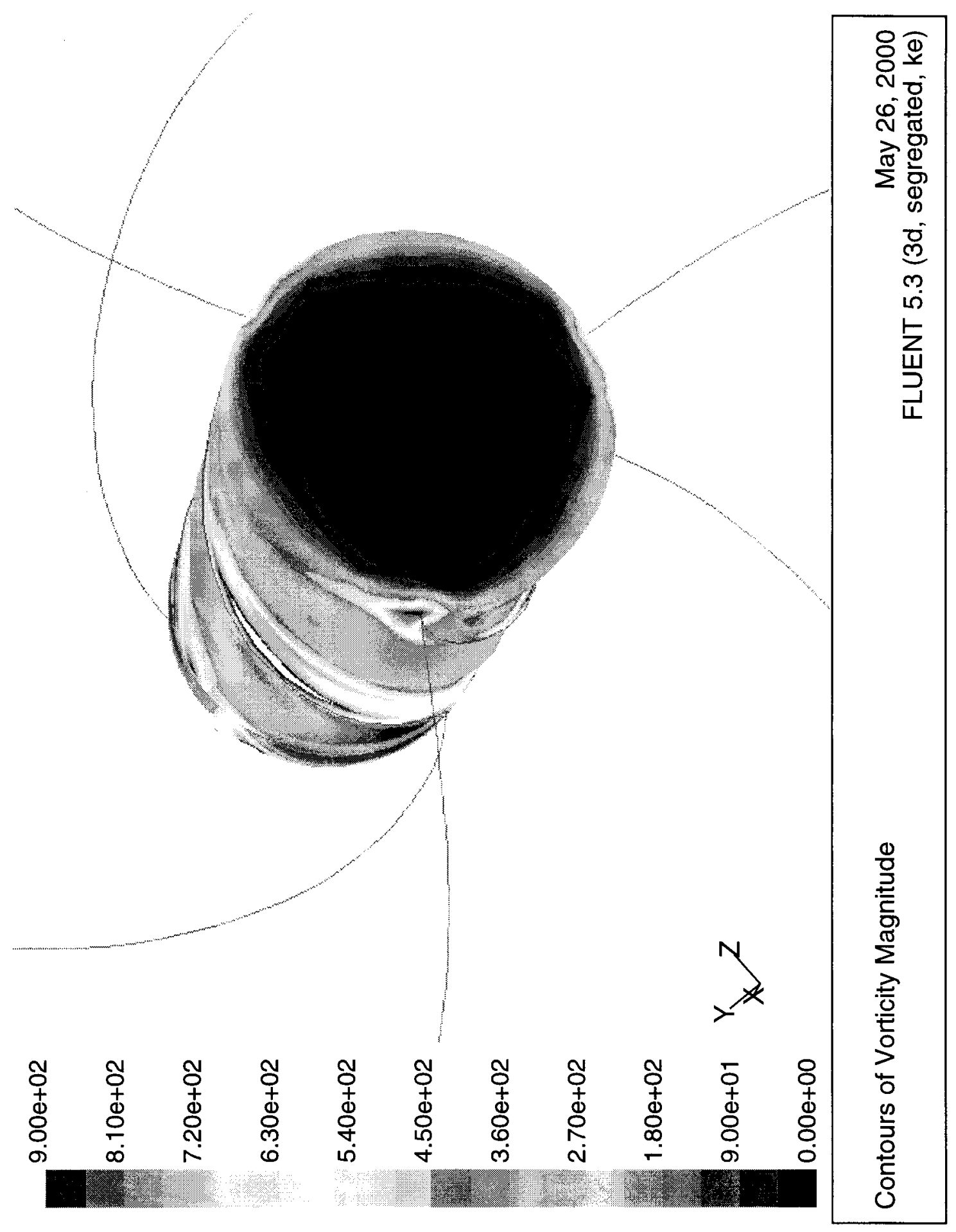

Figure 26. Vorticity contour plot at the propeller hub region (counterclockwise propeller direction). 


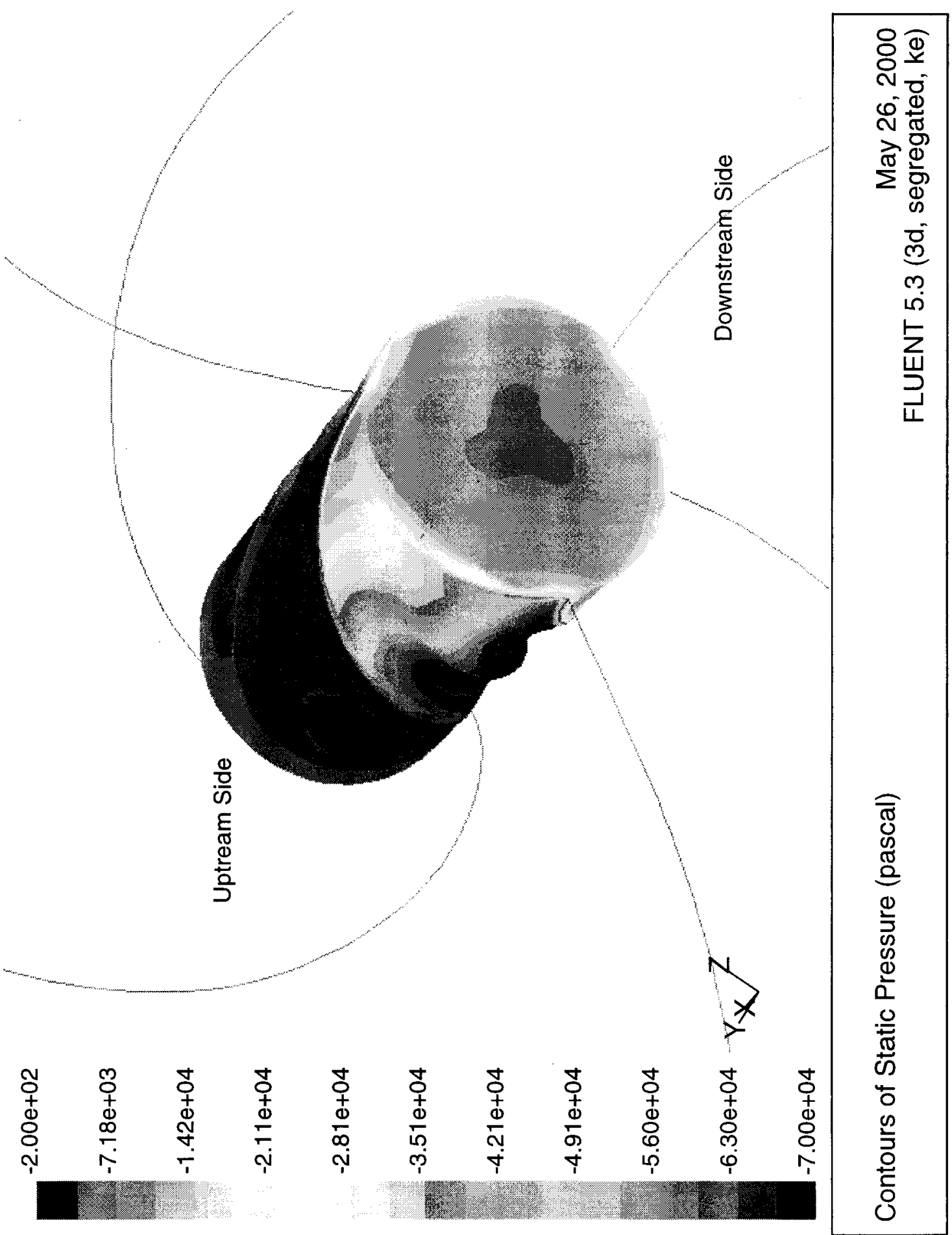

Figure 27. Static pressure contour plot at the propeller hub region (counterclockwise propeller direction). 


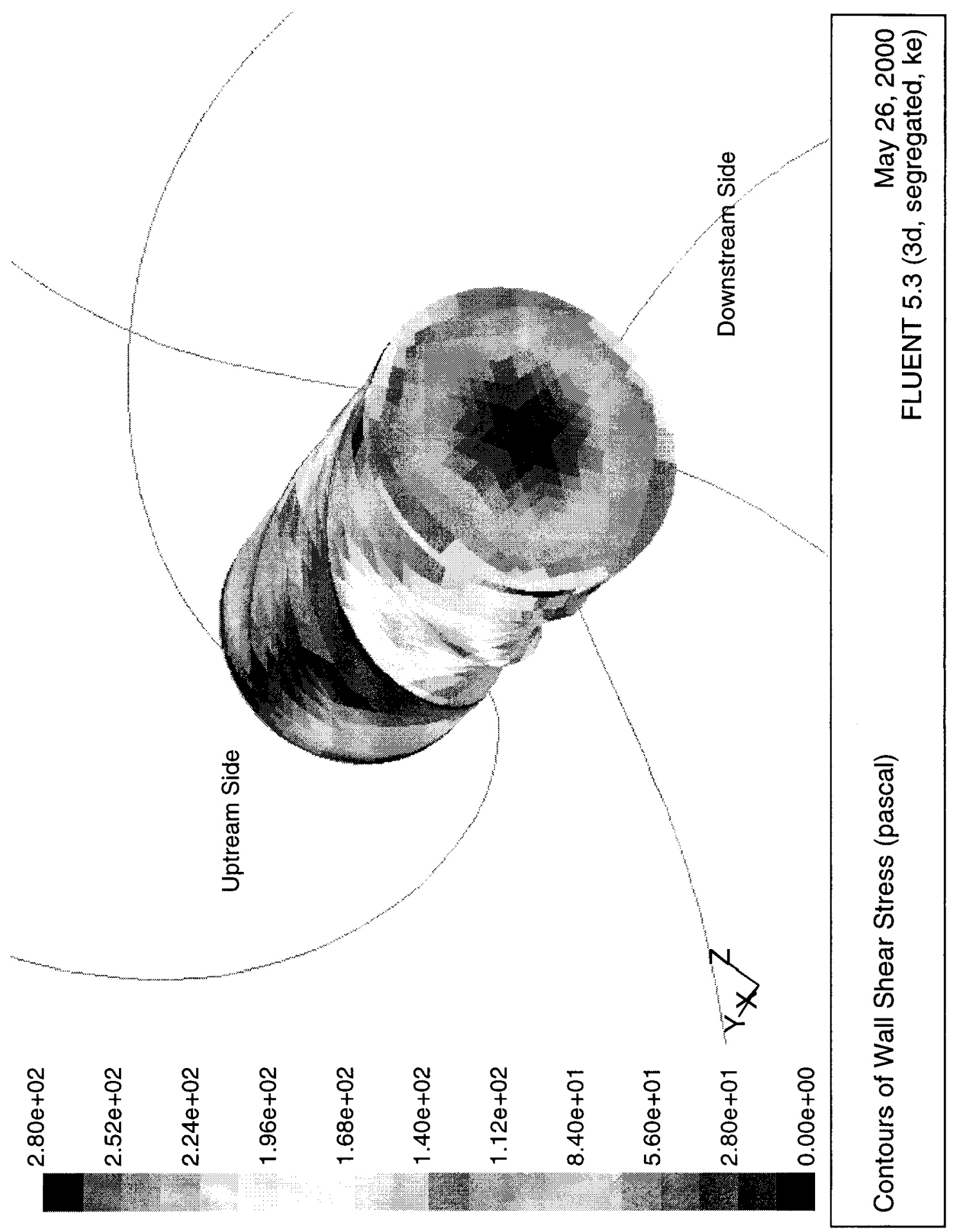

Figure 28. Shear stress contour plot at the propeller hub region (counterclockwise propeller direction). 


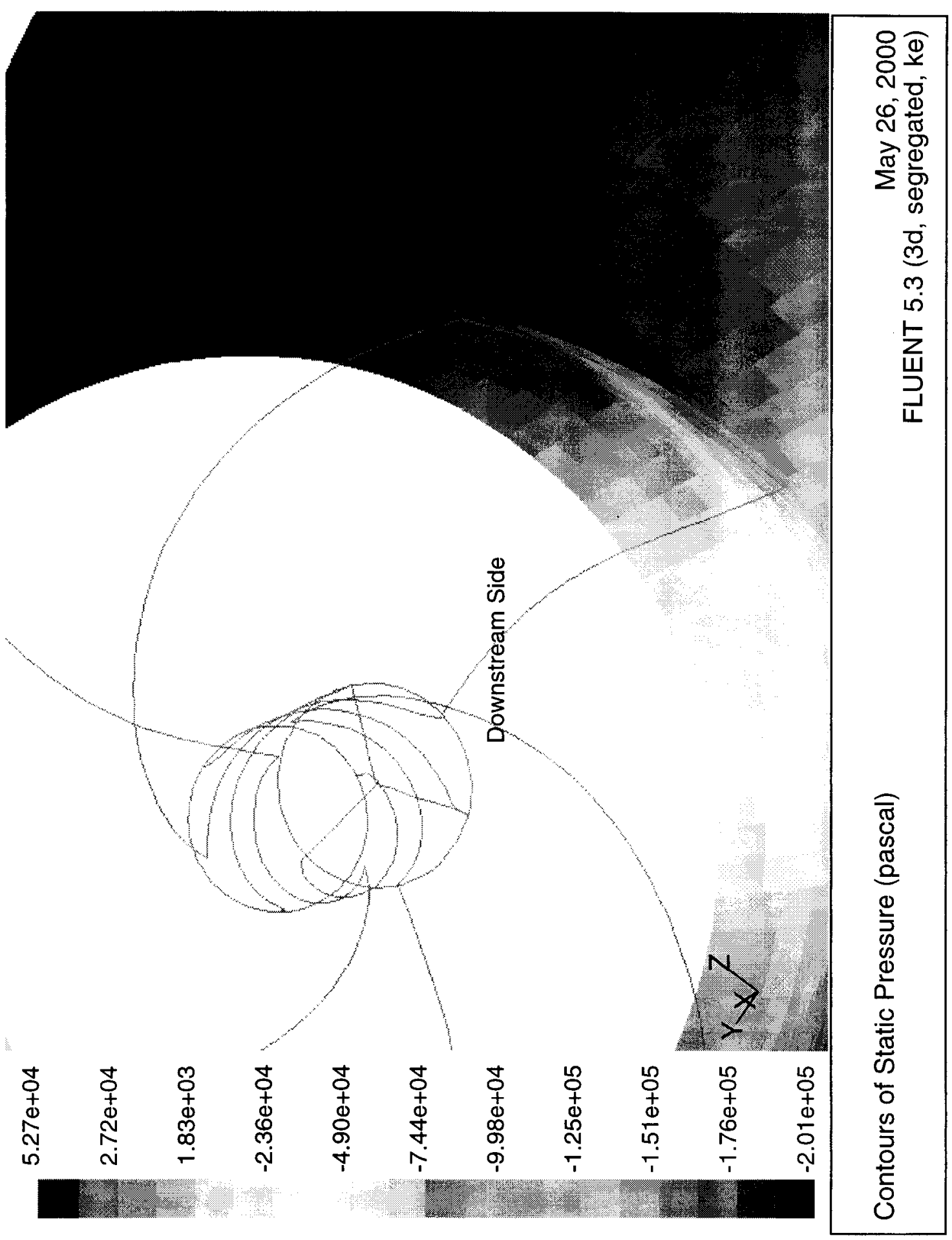

Figure 29. Static pressure contour plot at the shroud wall (counterclockwise propeller direction). 


$\begin{array}{lll}\text { Report: } & \text { WSRC-TR-2000-00205 WESTINGHOUSE SAVANNAH RIVER COMPANY } \\ \text { Date: } & 04 / 22 / 02\end{array}$

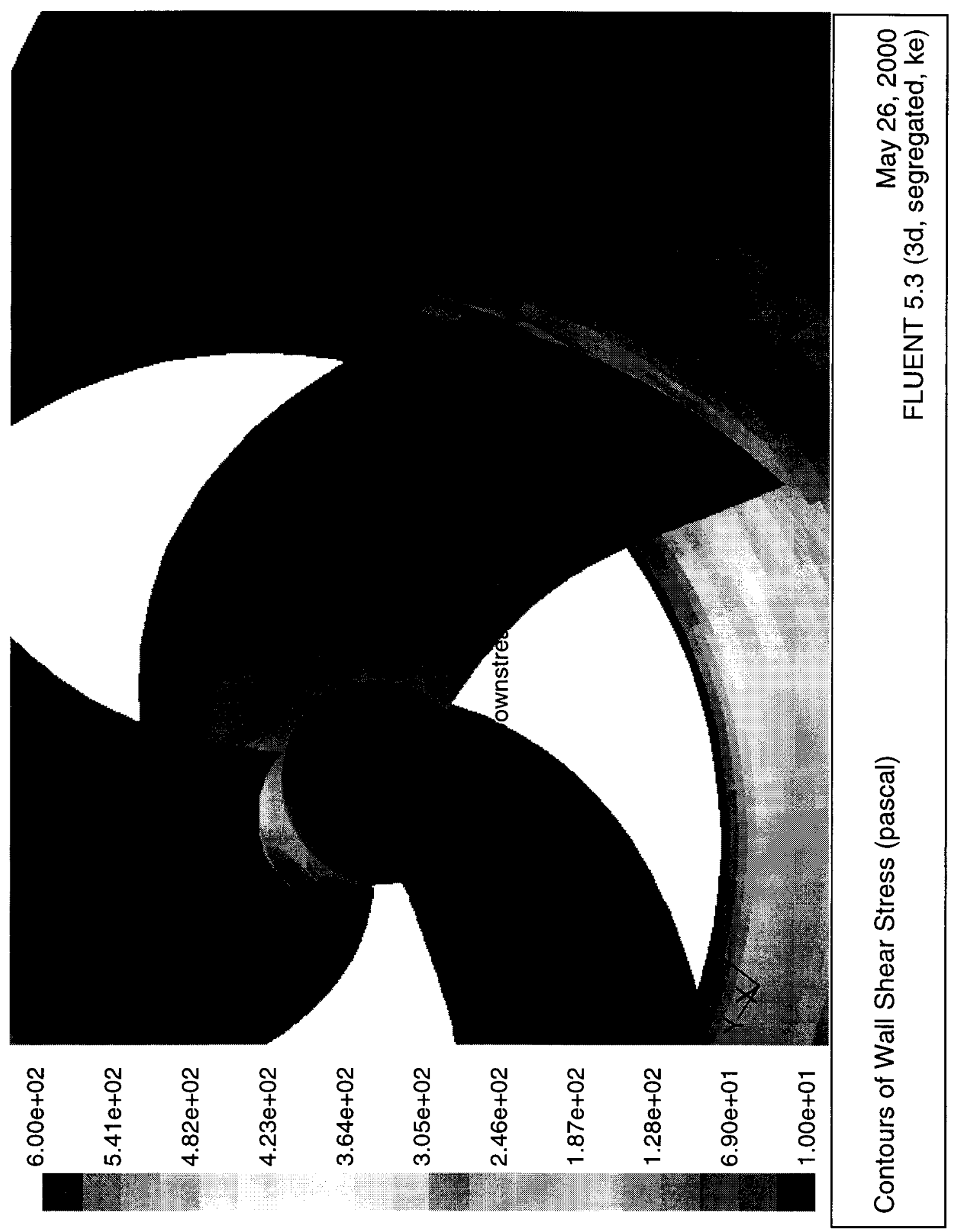

Figure 30. Shear stress contour plot at the shroud wall (counterclockwise propeller direction). 


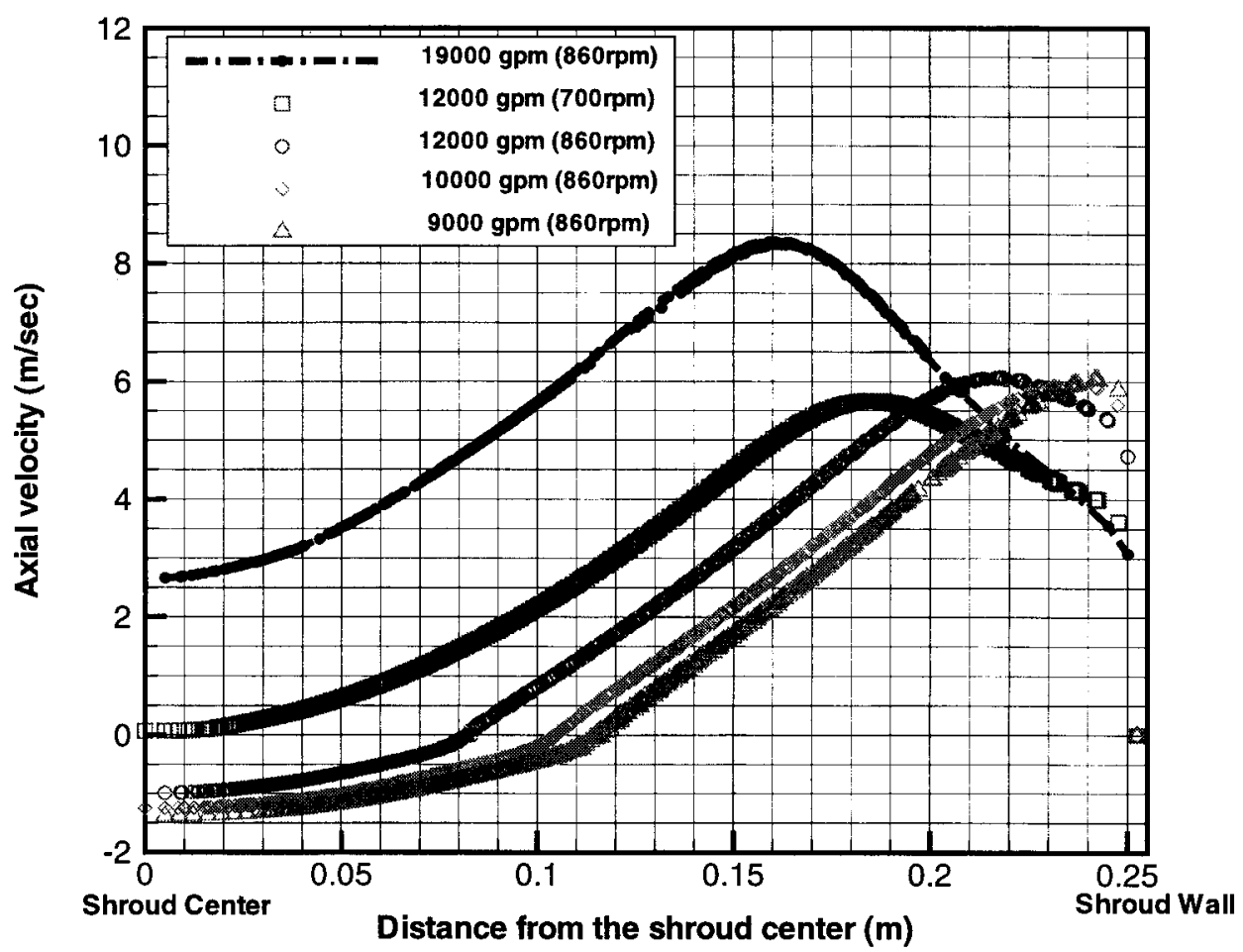

Figure 31. Velocity distributions along the radial direction. 


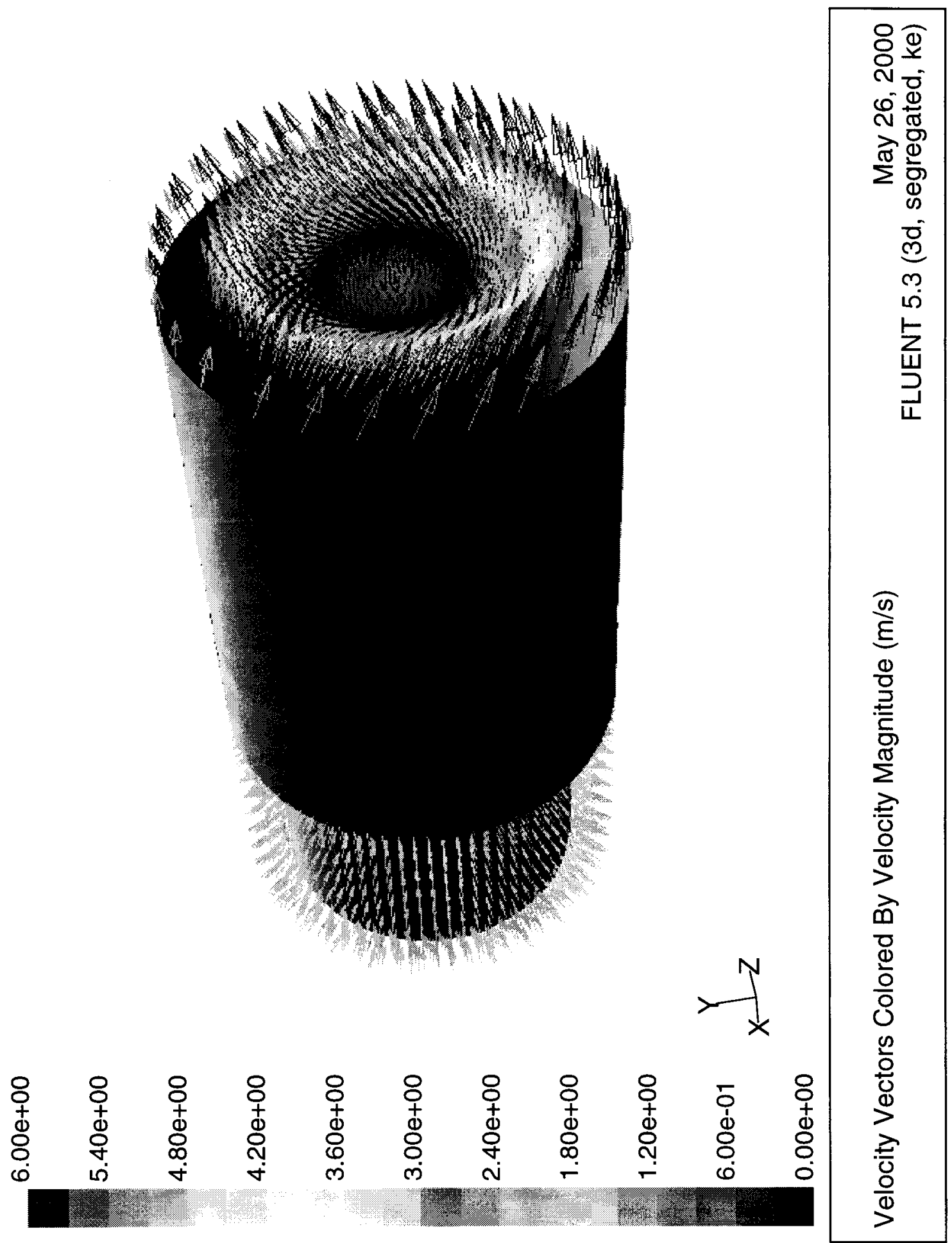

Figure 32. Velocity vector plot at the inlet and exit planes of shroud exit. 


\subsection{Sensitivity Run Results}

Sensitivity runs were performed by using the steady-state three-dimensional CFD model. The primary purpose is to investigate what physical parameters have the most impact on operating performance and system stability of the FLYGT mixer. From the steady-state model results, physical parameters such as inlet flow conditions, shroud wall roughness, and rotational speed of pump were chosen for the sensitivity studies.

- Non-uniform Inlet Flow Condition

This situation was simulated to examine the impact on the shroud exit velocity of nonuniform inlet velocity for the flow blockage caused by the motor housing upstream of the pump propeller. Non-uniform velocity was tested with an assumption that velocity around motor housing increased linearly along the flow direction toward the propeller region. That is,

$$
\begin{aligned}
& u_{\text {avg }}=\frac{1}{A} \int_{A_{1}}^{A_{2}} u_{r}(x) d A \\
& =\frac{1}{L_{\text {in }}} \int_{x=0}^{x=L_{\text {in }}}(\alpha+\beta x) d x
\end{aligned}
$$

Constants $\alpha$ and $\beta$ in the above integral, eq. (10), were determined by using the boundary condition $u_{r}(x=0)=0$ and the flow continuity $\mathrm{Q}$ (total volumetric flowrate) $=u_{\text {avg }} A$.

$u_{a v g}$ is area-averaged radial velocity at the inlet of the mixer. The area-averaged speeds for potential flow conditions are shown in Table 2. Detailed flow direction and notations used in the above equation are shown in Fig. 33. This non-uniform condition was based on the assumption that azimuthal velocity gradients at both sides of the symmetry planes in $120^{\circ}$ sector geometry of the mixer, as shown in Fig. 3, are zero. For these studies, typical operating conditions were chosen as two different operating flows (9,000 and $12,000 \mathrm{gpm}$ ) with $860 \mathrm{rpm}$ pump speed.

The results showed that at shroud exit radial flow distributions for non-uniform inlet condition is a little bit more uniform than those of uniform inlet flow condition. The sensitivity results are shown in Fig. 34. Total loads for the three components of the mixer with non-uniform inlet flow are about $7 \%$ lower than those of uniform inlet flow for the operating conditions of $12,000 \mathrm{gpm}$ flow with $860 \mathrm{rpm}$ pump speed. Three-dimensional velocity flow pattern near the inlet and the shroud exit regions is shown in Fig. 35. However, for 9,000 gpm pump flow with $860 \mathrm{rpm}$ pump speed, loads for non-uniform inlet flow are close to those of uniform inlet flow. The detailed comparison of the two cases is shown in Table 7. 


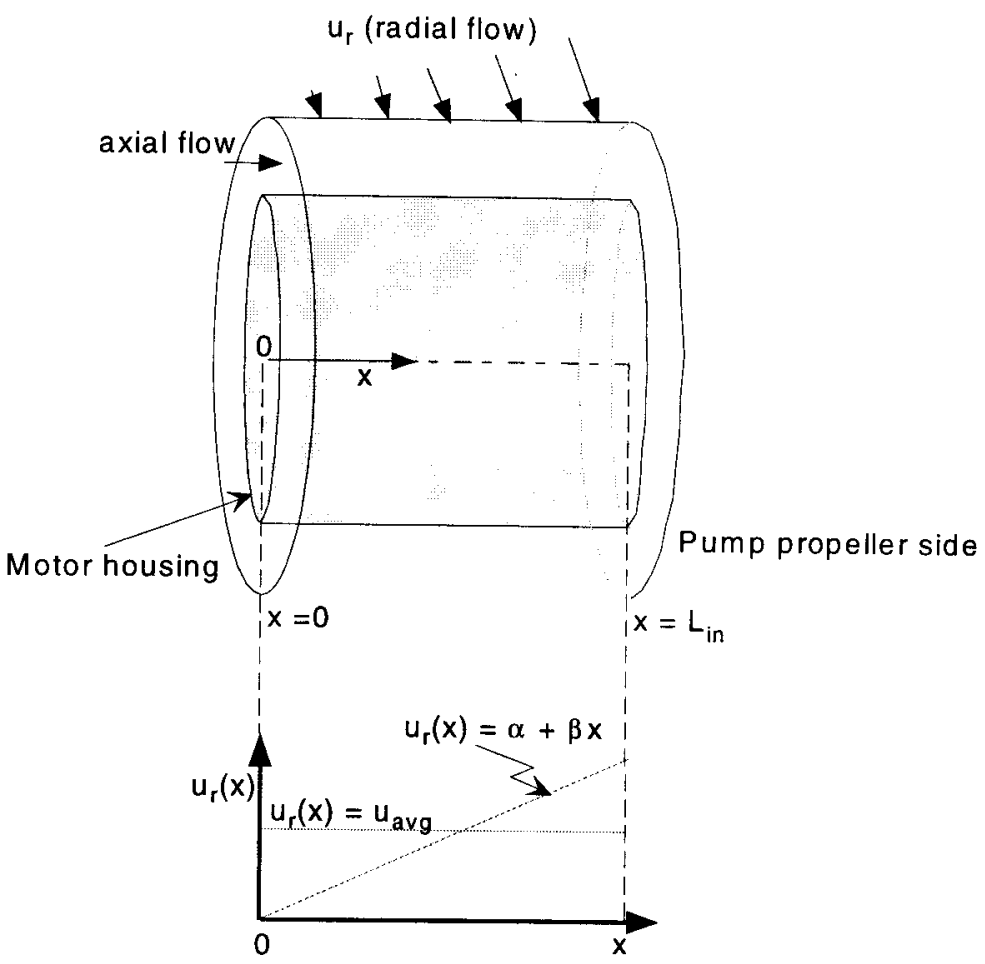

Figure 33. Non-uniform and uniform flow conditions at the pump inlet for the sensitivity runs.

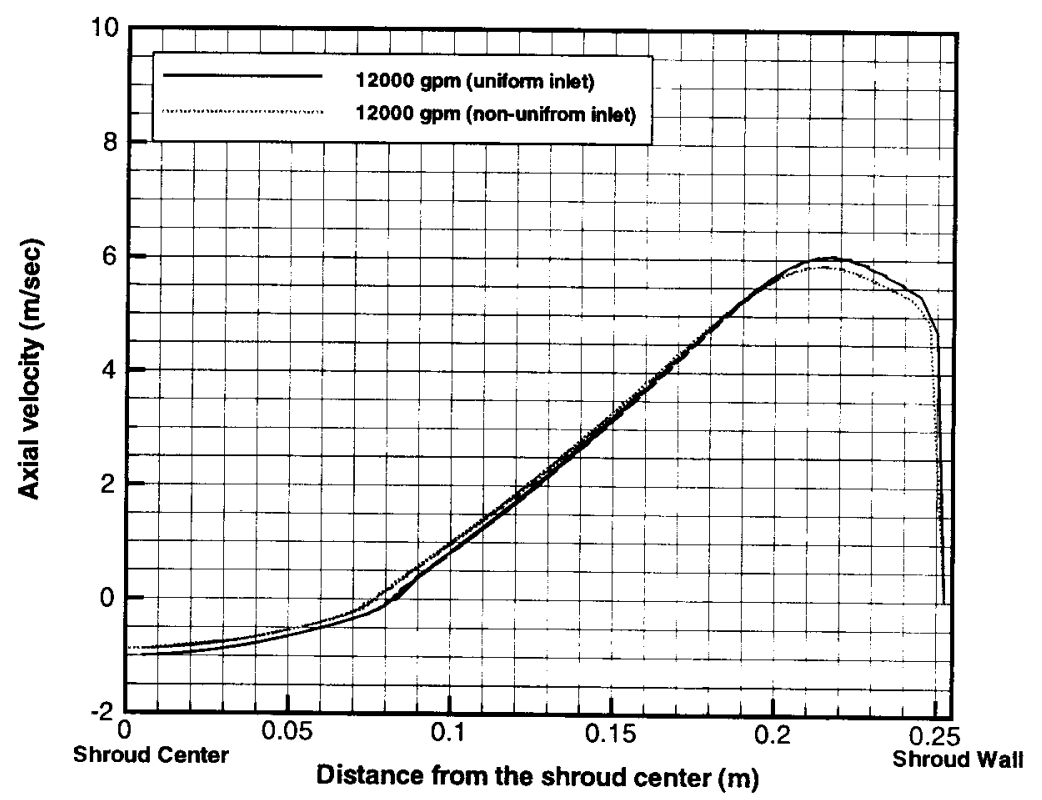

Figure 34. Sensitivity run results for axial flow velocities at shroud exit for $12,000 \mathrm{gpm}$ pump flow and $860 \mathrm{rpm}$ pump speed. 
Table 7. Influence of inlet flow non-uniformity on the axial loads of pump blade, shaft, and shroud wall regions under steady-state operating conditions of two different flows with $860 \mathrm{rpm}$ pump speed.

\begin{tabular}{|c|c|c|c|c|c|}
\hline \multirow{2}{*}{$\begin{array}{l}\text { Pump flow } \\
\text { (gpm) }\end{array}$} & \multirow{2}{*}{$\begin{array}{l}\text { Shroud wall } \\
\text { surface } \\
\text { conditions }\end{array}$} & \multirow{2}{*}{$\begin{array}{l}\text { Loads by } \\
\text { hydraulics }\end{array}$} & \multicolumn{3}{|c|}{ Components } \\
\hline & & & $\begin{array}{c}\text { Blade } \\
\text { (pound-force) }\end{array}$ & $\begin{array}{c}\text { Shaft } \\
\text { (pound-force) }\end{array}$ & $\begin{array}{c}\text { Shroud } \\
\text { (pound-force) }\end{array}$ \\
\hline \multirow{4}{*}{9,000} & \multirow{2}{*}{$\begin{array}{c}\text { Uniform inlet } \\
\text { flow }\end{array}$} & Pressure & -1645.3 & 20.7 & -7.9 \\
\hline & & Viscous & 4.8 & -0.1 & 6.0 \\
\hline & \multirow{2}{*}{$\begin{array}{l}\text { Non-uniform } \\
\text { inlet flow }\end{array}$} & Pressure & -1639.1 & 19.5 & -7.8 \\
\hline & & Viscous & 4.8 & -0.1 & 6.0 \\
\hline \multirow{4}{*}{12,000} & \multirow{2}{*}{$\begin{array}{c}\text { Uniform inlet } \\
\text { flow }\end{array}$} & Pressure & -1406.3 & 25.0 & -8.8 \\
\hline & & Viscous & 4.4 & -0.6 & 4.2 \\
\hline & \multirow{2}{*}{$\begin{array}{l}\text { Non-uniform } \\
\text { inlet flow }\end{array}$} & Pressure & -1309.6 & 23.7 & -8.2 \\
\hline & & Viscous & 4.3 & -0.5 & 3.9 \\
\hline
\end{tabular}

\section{- Shroud Wall Roughness}

One of the major concerns is the structural vibration and sturdiness associated with loading due to the high-speed pump operation. As mentioned earlier, shroud design was recently modified and tested at the TNX full tank facility (Adkins, 2000). The modifications were made in response to multiple mechanical failures. A major modification was a new, single-piece, machined shroud to improve mixer reliability. With the new geometry, loading sensitivity of the shroud needs to be examined based on the surface roughness of the shroud inner wall being smooth or rough. A rough wall condition was simulated as a concrete surface whose roughness height was $0.01 \mathrm{ft}$ (Rohsenow and Choi, 1961). Maximum velocity was decreased by about $4 \%$ due to turbulence increase compared to the smooth surface. Load of the shroud was increased by about $2 \%$ compared to the smooth surface of the shroud wall. Results are compared between two cases in Table 8 . The graphical results are shown in Fig. 36.

Table 8. Influence of shroud wall roughness on the axial loads of pump blade, shaft, and shroud wall regions under $9,000 \mathrm{gpm}$ flow and $860 \mathrm{rpm}$ pump speed of steadystate operating condition.

\begin{tabular}{|c|c|c|c|c|}
\hline \multirow{2}{*}{$\begin{array}{l}\text { Shroud wall surface } \\
\text { conditions }\end{array}$} & \multirow{2}{*}{$\begin{array}{l}\text { Loads by } \\
\text { hydraulics }\end{array}$} & \multicolumn{3}{|c|}{ Components } \\
\hline & & $\begin{array}{c}\text { Blade } \\
\text { (pound-force) }\end{array}$ & $\begin{array}{c}\text { Shaft } \\
\text { (pound-force) }\end{array}$ & $\begin{array}{c}\text { Shroud } \\
\text { (pound-force) }\end{array}$ \\
\hline \multirow{2}{*}{ Smooth surface } & Pressure & -1645.3 & 20.7 & -7.9 \\
\hline & Viscous & 4.8 & -0.1 & 6.0 \\
\hline \multirow{2}{*}{$\begin{array}{l}\text { Rough surface } \\
\text { (roughness }=0.01 \mathrm{ft} \text { ) }\end{array}$} & Pressure & -1677.5 & 20.5 & -8.3 \\
\hline & Viscous & 4.7 & -0.1 & 19.4 \\
\hline
\end{tabular}


Report:
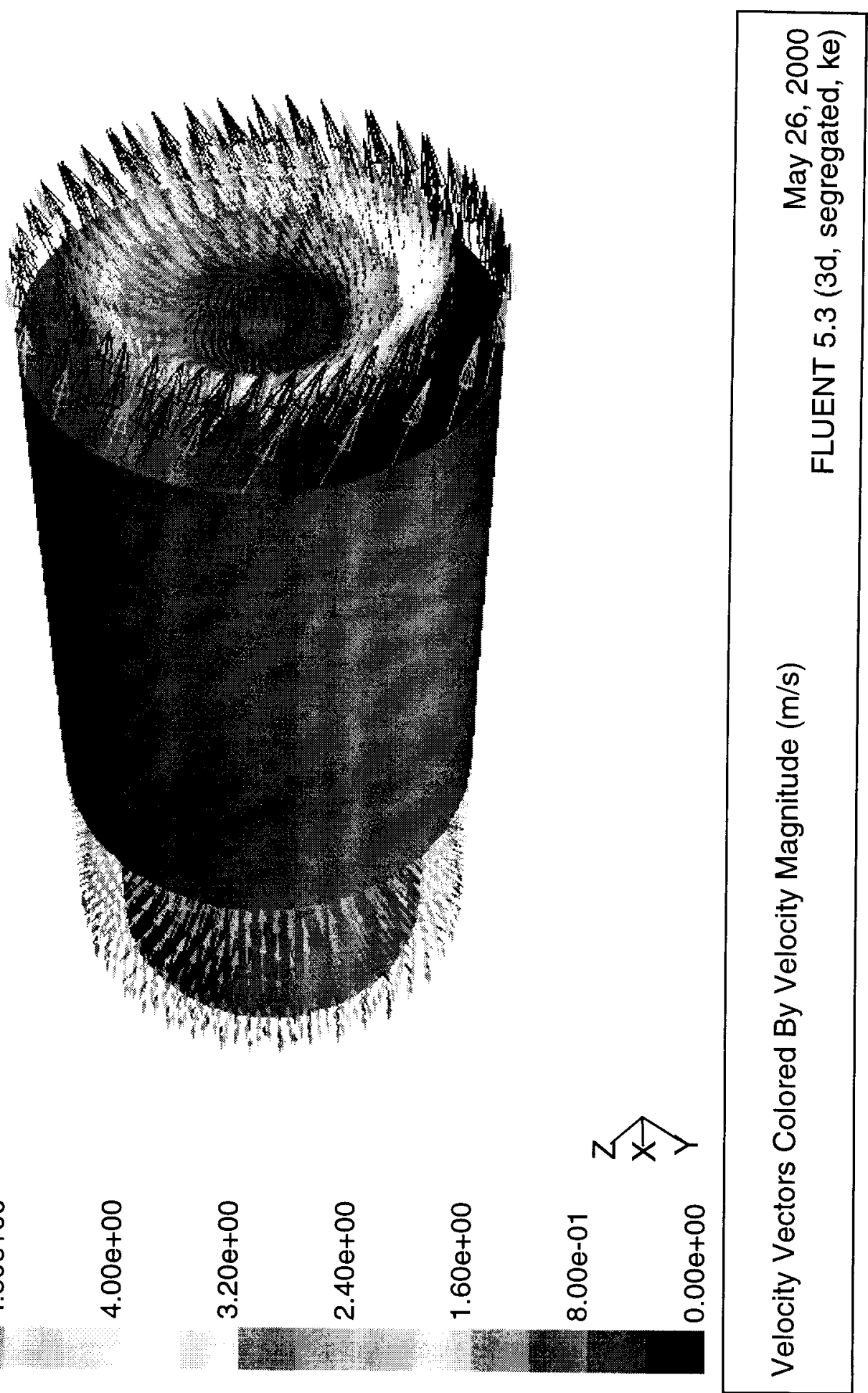

Figure 35. Velocity vector plot at the inlet and exit planes for non-uniform inlet flow condition. 


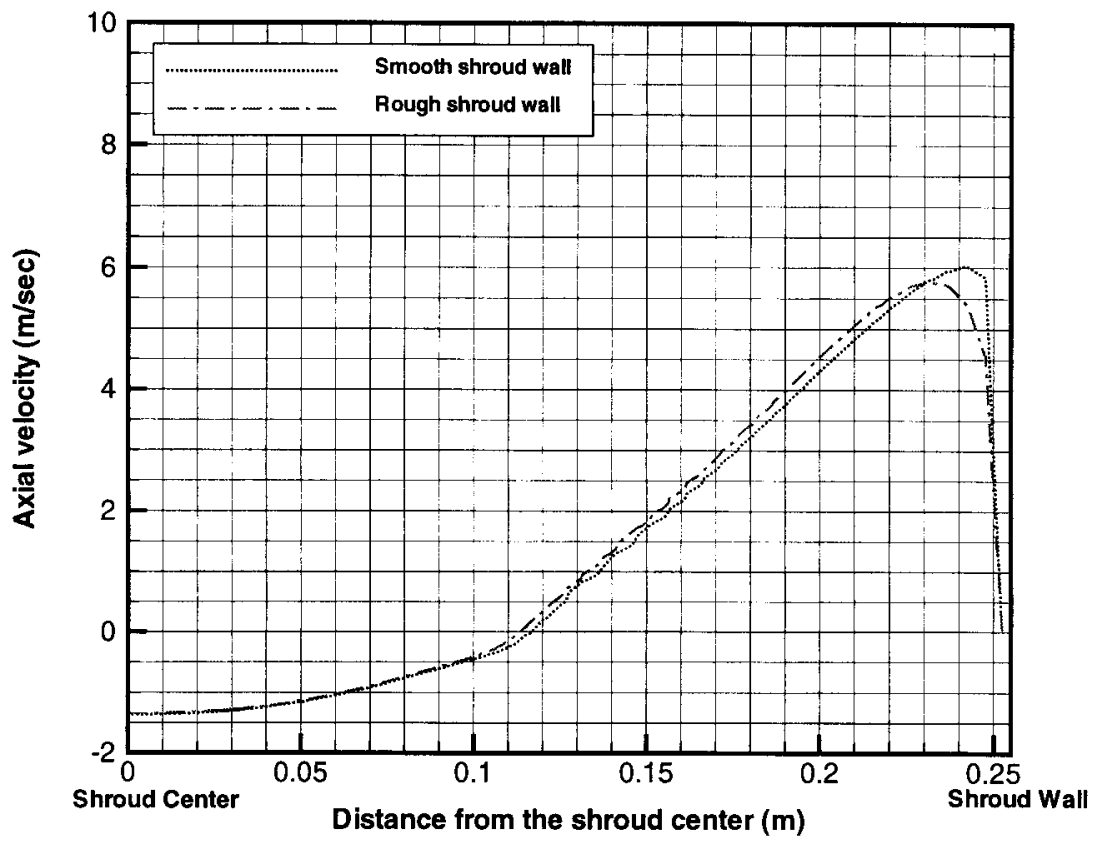

Figure 36. Sensitivity run results for surface roughness effects of shroud wall on axial flow velocities at shroud exit (Roughness height of rough wall used in this figure was $0.01 \mathrm{ft}$ equivalent to concrete surface.).

\section{- Pump Rotational Speed}

From the TNX test (Adkins, 2000) of the modified design, as mentioned earlier, overall vibration levels on the motor and shroud were high compared to industry standards for rotating machines such as pumps. Vibration testing with variable speed revealed a dramatic increase ( 3 to 4 times) in motor and shroud vibration between 700 and $860 \mathrm{rpm}$. A pump speed of $860 \mathrm{rpm}$ corresponds to about 42 periodic cycles. This large increase in vibration appears to be the result of a system natural frequency (about 40 periodic cycles). The model predicted that the maximum axial velocity for $700 \mathrm{rpm}$ at the shroud exit was slightly decreased compared to that of $860 \mathrm{rpm}$ as shown in Fig. 37. However, loads on the pump were found to be very sensitive to the rotational speeds of the pump. Table 9 shows the model results for 500 to $860 \mathrm{rpm}$ ranges of the motor speed. These results are qualitatively consistent with the HLW test results (Adkins, 2000) in a qualitative way.

Table 10 summarizes typical sensitivity results obtained from the present study. As expected, the results showed that pump speed is the most sensitive parameter among the three parameters in terms of loading change for the pump system during steady-state operation. 


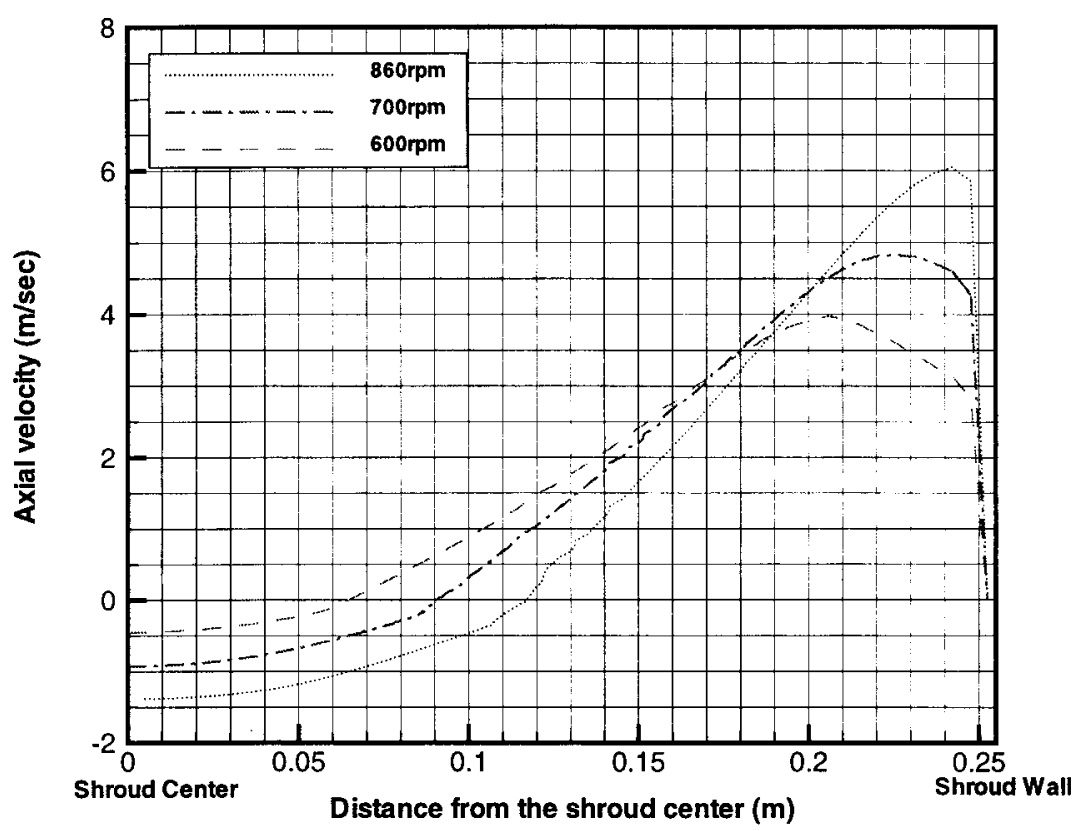

Figure 37. Sensitivity run results for pump speed effects on axial flow velocities at the exit of the pump shroud under 9,000 gpm flow and $860 \mathrm{rpm}$ pump speed.

Table 9. Influence of rotational pump speed on the axial loads of pump blade, shaft, and shroud wall regions under $9,000 \mathrm{gpm}$ flow of steady-state operating condition in a smooth shroud wall.

\begin{tabular}{|c|c|c|c|c|}
\hline \multirow{2}{*}{$\begin{array}{l}\text { Pump rotational } \\
\text { speed } \\
\text { (rpm) }\end{array}$} & \multirow{2}{*}{$\begin{array}{l}\text { Loads by } \\
\text { hydraulics }\end{array}$} & \multicolumn{3}{|c|}{ Components } \\
\hline & & $\begin{array}{c}\text { Blade } \\
\text { (pound-force) }\end{array}$ & $\begin{array}{c}\text { Shaft } \\
\text { (pound-force) }\end{array}$ & $\begin{array}{c}\text { Shroud } \\
\text { (pound-force) }\end{array}$ \\
\hline \multirow{2}{*}{500} & Pressure & -483.9 & 7.8 & -3.6 \\
\hline & Viscous & 1.9 & -0.5 & -0.4 \\
\hline \multirow{2}{*}{600} & Pressure & -651.1 & 12.9 & -4.2 \\
\hline & Viscous & 2.3 & -0.3 & 1.8 \\
\hline \multirow{2}{*}{700} & Pressure & -909.6 & 16.9 & -5.7 \\
\hline & Viscous & 3.1 & -0.4 & 2.8 \\
\hline \multirow{2}{*}{860} & Pressure & -1645.3 & 20.7 & -7.9 \\
\hline & Viscous & 4.8 & -0.1 & 6.0 \\
\hline
\end{tabular}


Table 10. Summary of load sensitivities to the changes of the physical parameters for the propeller blade for $9000 \mathrm{gpm}$ flow and $860 \mathrm{rpm}$ operating condition (Negative number means decrease in load.).

\begin{tabular}{|c|c|c|c|c|}
\hline \multirow{2}{*}{ Parameters } & $\begin{array}{c}\text { Change of inlet flow } \\
\text { distribution }\end{array}$ & $\begin{array}{c}\text { Roughness change } \\
\text { of shroud wall } \\
\text { surface }\end{array}$ & \multicolumn{2}{|c|}{$\begin{array}{c}\text { Change of rotational pump } \\
\text { speed (rpm) }\end{array}$} \\
\cline { 2 - 5 } & (from uniform to non-uniform) & (from smooth to rough ${ }^{*}$ ) & from 600 to 860 & From 700 to 860 \\
\hline $\begin{array}{c}\text { Relative } \% \\
\text { change }\end{array}$ & $<-1 \%$ & $+2 \%$ & $+153 \%$ & $+81 \%$ \\
\hline
\end{tabular}

Note: *Rough surface corresponds to rough concrete surface (0.01ft of roughness height (Rohsenow and Choi (1961)).

\subsection{Transient Flow Behavior of FLYGT Mixer Associated with Pump Cavitation}

Cavitation is generally caused by fast-moving bodies in liquid, either with a free surface (propeller) or in closed conduits (pump impeller). The word "cavitation" is used in the literature to signify either the hydrodynamic phenomenon of the formation of vapor-filled bubbles or cavities at low pressures and the subsequent disappearance of such bubbles, or the physical damage to materials which form the boundaries of the fluid passages in which this bubble formation occurs and it is carried away by the liquid phase.

In this report, attention is limited to the hydraulically controlled vapor generation phenomenon. That is, vapor bubble formation is caused by pressure reduction within the water at room temperature. By this usage, an object such as a pump blade is said to cavitate if such vapor bubbles are formed, even though no physical damage occurs. It is assumed that cavitation will occur whenever the pressure at some point in the fluid becomes equal to the vapor pressure. Local boiling results in vapor-filled cavities which grow so long as they are in a low-pressure environment. Assuming the inception of cavitation when the pressure equals the vapor pressure exactly implies that the fluid will not support a tension and ignores the possibility of dissolved gases being released to cause premature cavitation at pressures higher than the vapor pressure. Nevertheless, there is considerable experimental information to indicate that with water containing ordinary amounts of impurities and dissolved air, cavitation does occurs at pressures that are very close to the vapor pressure.

In the literature experiment (Wood, 1963), the cavitation formations in the vane channels of the pump impellers were observed to be cyclic in nature, whereas the cavitation near the leading edge of the blade was more stable. It was noted that the observed cyclic behavior might be the principal source of noise and vibration in cavitating hydraulic machinery. The cavitation formations associated with loss of hydraulic performance were cyclic in nature. The cavitation was observed to occur initially near the tip leading edge of the blades for the axial mixed flow pump impellers tested (Wood, 1963). Thus, cavitation initiated by the tip vortex may be the principal cause of performance deterioration. These 
phenomena are consistent with the present CFD results as shown in the subsequent figures.

The primary objective of the transient cavitation model is to evaluate the changes in nominal loads introduced by a sudden change in fluid density from the cavitation development near the tip of the propeller blade and to investigate the time-dependent behavior of the high-speed mixer performance. As mentioned earlier, the bubble condensation effect in the model was neglected since bubble residence time in the 20" shroud was assumed to be very short and all cavitation bubbles inside the shroud remained tiny (less than $1 \mathrm{~mm}$ from the literature data) and bubbly flow. No slip velocity between the two phases was assumed.

All transient simulations in this report used $12,000 \mathrm{gpm}$ flowrate and $860 \mathrm{rpm}$ pump speed as pump operating condition in 20 " long, single-piece, smooth shroud. Steady-state results without interphase mass transfer due to cavitation effect were used as initial pump condition. Figure 38 shows the transient response of total velocity along the radial direction at the plane of shroud exit. Transient response of axial velocity along the radial direction at shroud exit, in relation to the pump performance, is shown in Fig. 39. Transient model results showed that transient flow velocity developed to steady-state condition within about 1 second, corresponding to about 40 cycles of the propeller blade after the initiation of cavitation.

Transient response of total pressure load for all the three blades of pump propeller are presented in Fig. 40. During initial period of transient time, the load on the blades increased rapidly and then was established to steady-state in about 1 second. Figure 41 shows transient loads of the hub region for $12,000 \mathrm{gpm}$ and $860 \mathrm{rpm}$ operating conditions. Transient response of the shroud load is shown in Fig. 42.

Transient flow behaviors of the FLYGT mixer such as fluid pressure, density distributions, fluid velocity, and vorticity are important in relation to the deterioration of pump performance and system stability since, in reality, fluid flow behavior is closely coupled with structural stability of the system such as pump vibration and noise. The results for the key fluid parameters related to the operating performance of the mixer are presented in Fig. 43 through 46 . The results showed that cavitation occurred due mainly to the fluid vortex near the leading-edge tip of the propeller blade. It is noted that a low density region was established near the peripheral region adjacent to the shroud wall because all vapor bubbles generated as a result of cavitation migrated to the high-velocity fluid region near the tip of the blade.

From the transient cavitation model, transient flow and loading behaviors of the mixer were examined for two different pump speeds (860 rpm and $700 \mathrm{rpm}$ ) under the same flow $(12,000 \mathrm{gpm})$ condition. The results showed that the transient time for $700 \mathrm{rpm}$ pump to reach steady-state condition is longer by about 0.5 second, corresponding to 20 cycles of the blade, than that of the $860 \mathrm{rpm}$ pump. The transient results for the velocity profiles at the shroud exit and the loading at the propeller blade are shown in Figs. 47 and 48. 


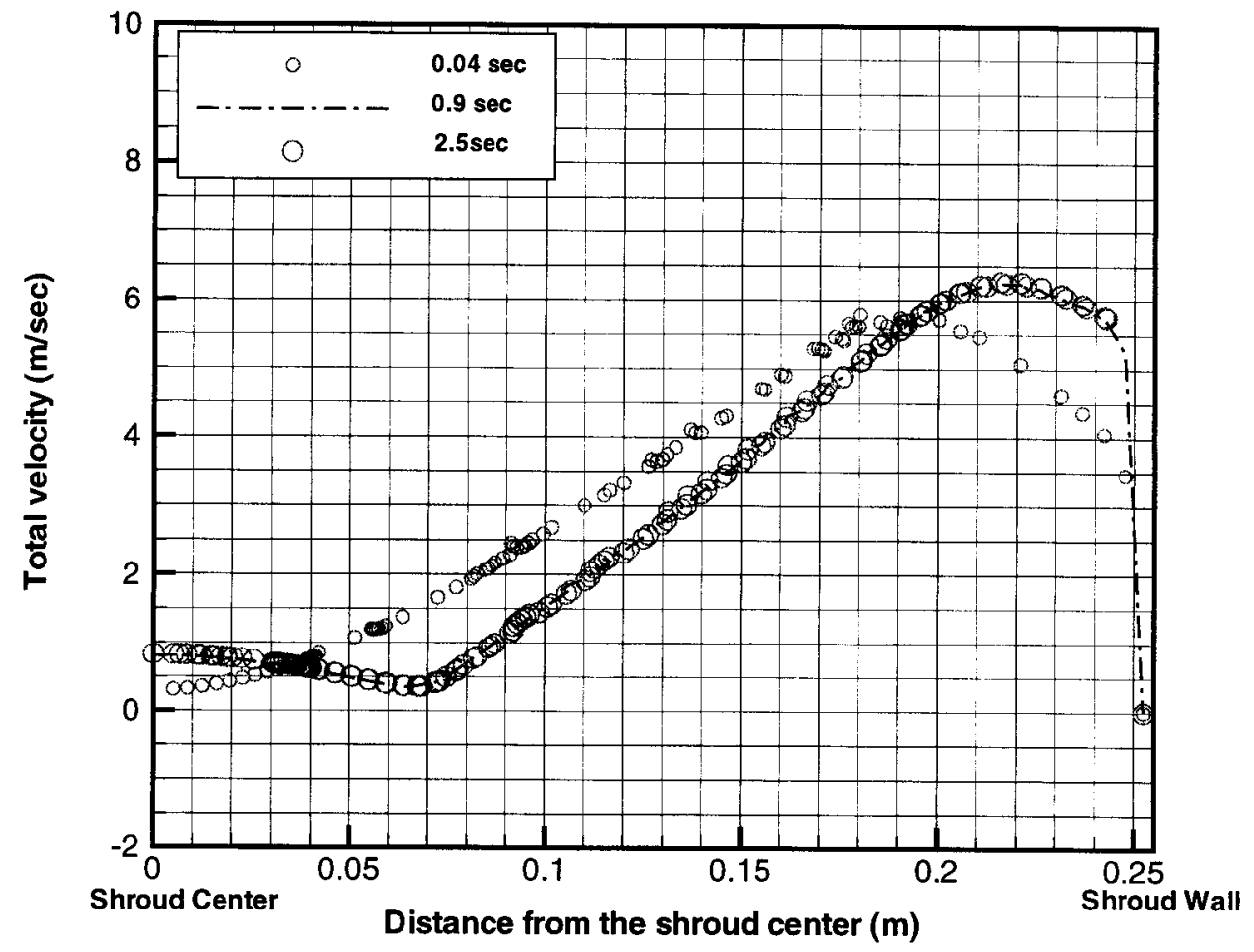

Figure 38. Transient response of flow velocity along the radial direction of the shroud exit plane for 12,000 gpm and $860 \mathrm{rpm}$ operating condition. 
Report:

Date:

Page:

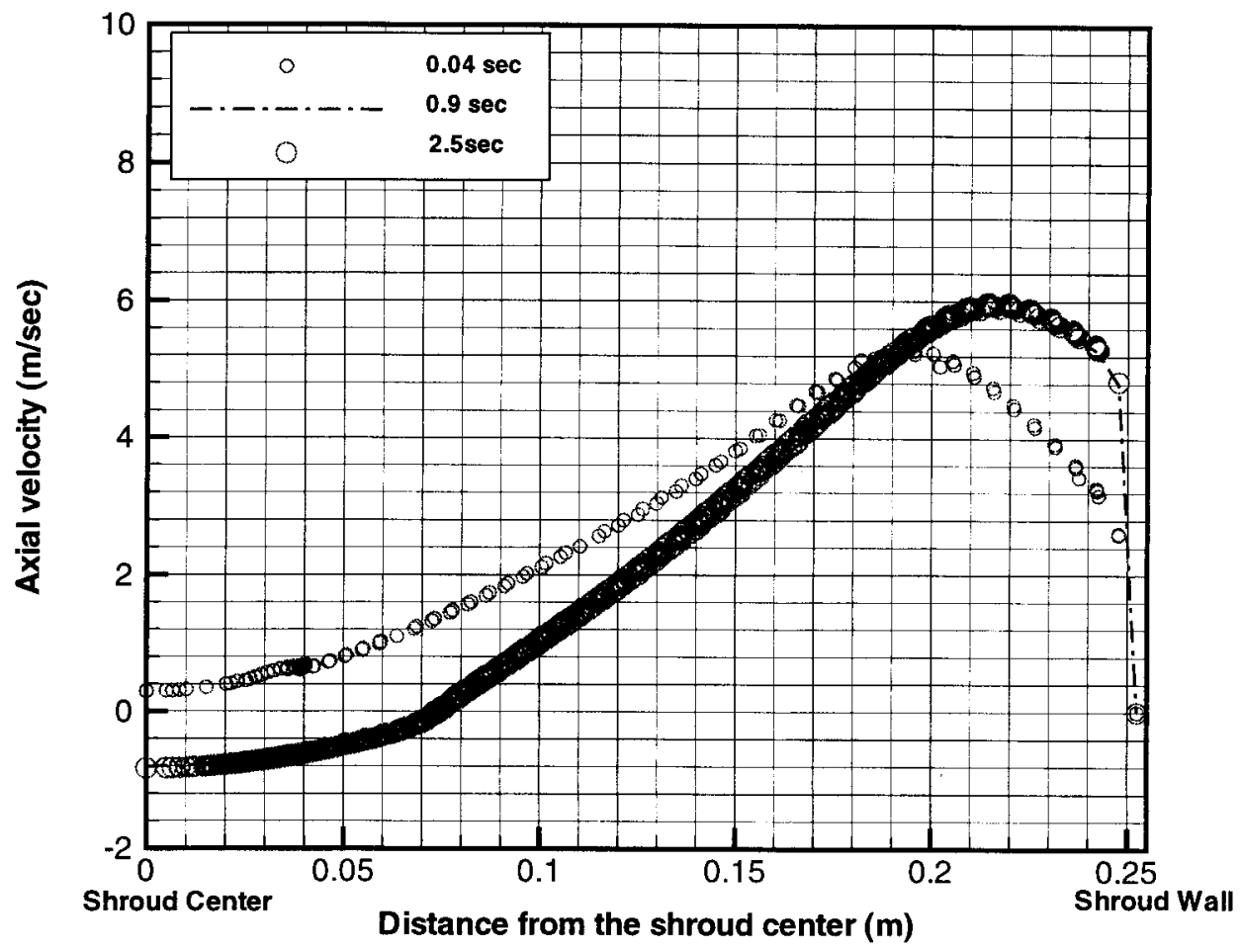

Figure 39. Transient response of axial flow velocity along the radial direction of the shroud exit plane for $12,000 \mathrm{gpm}$ and $860 \mathrm{rpm}$ operating condition. 


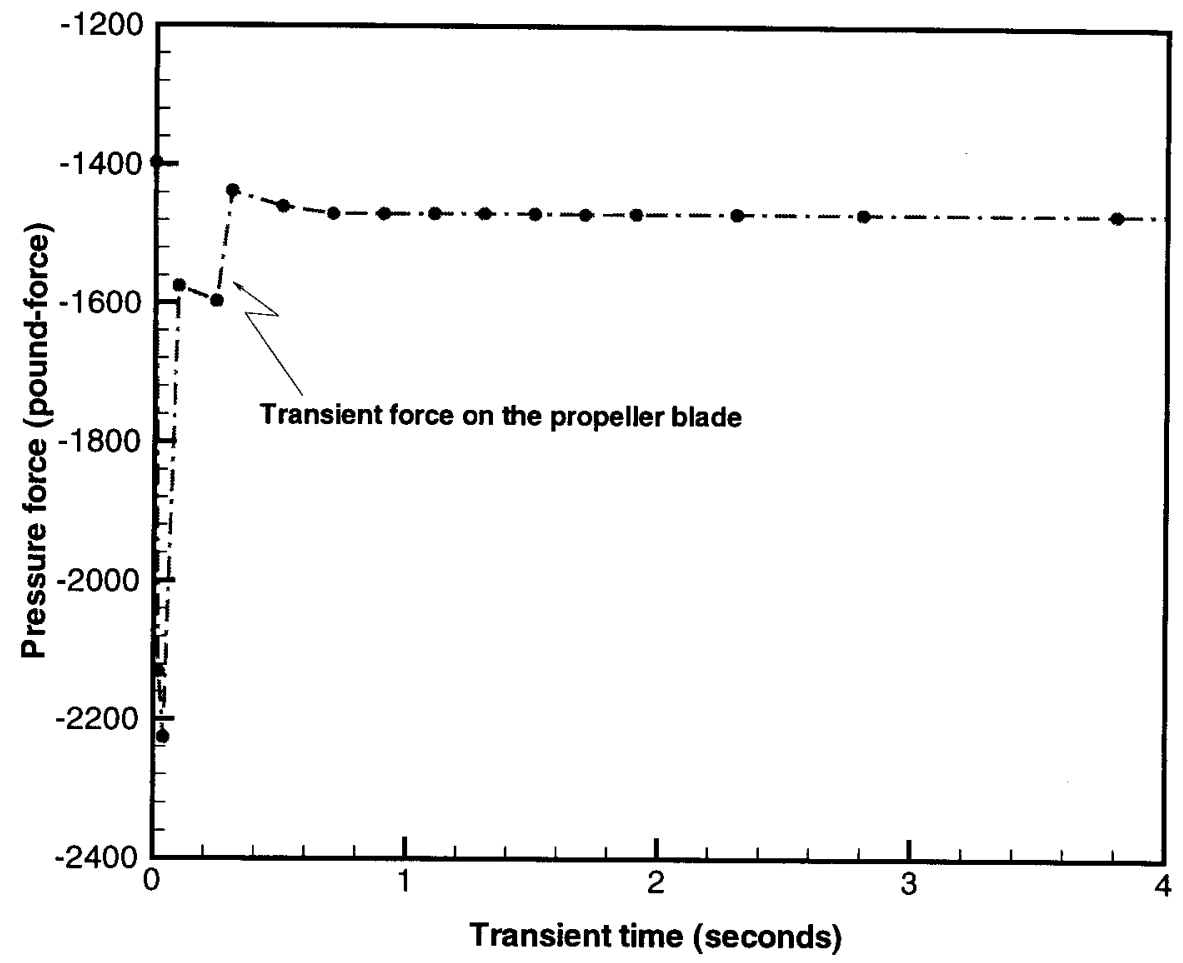

Figure 40. Transient response of total pressure force for three propeller blades under $12,000 \mathrm{gpm}$ and $860 \mathrm{rpm}$ operating condition. 
Report:

Date:

Page:

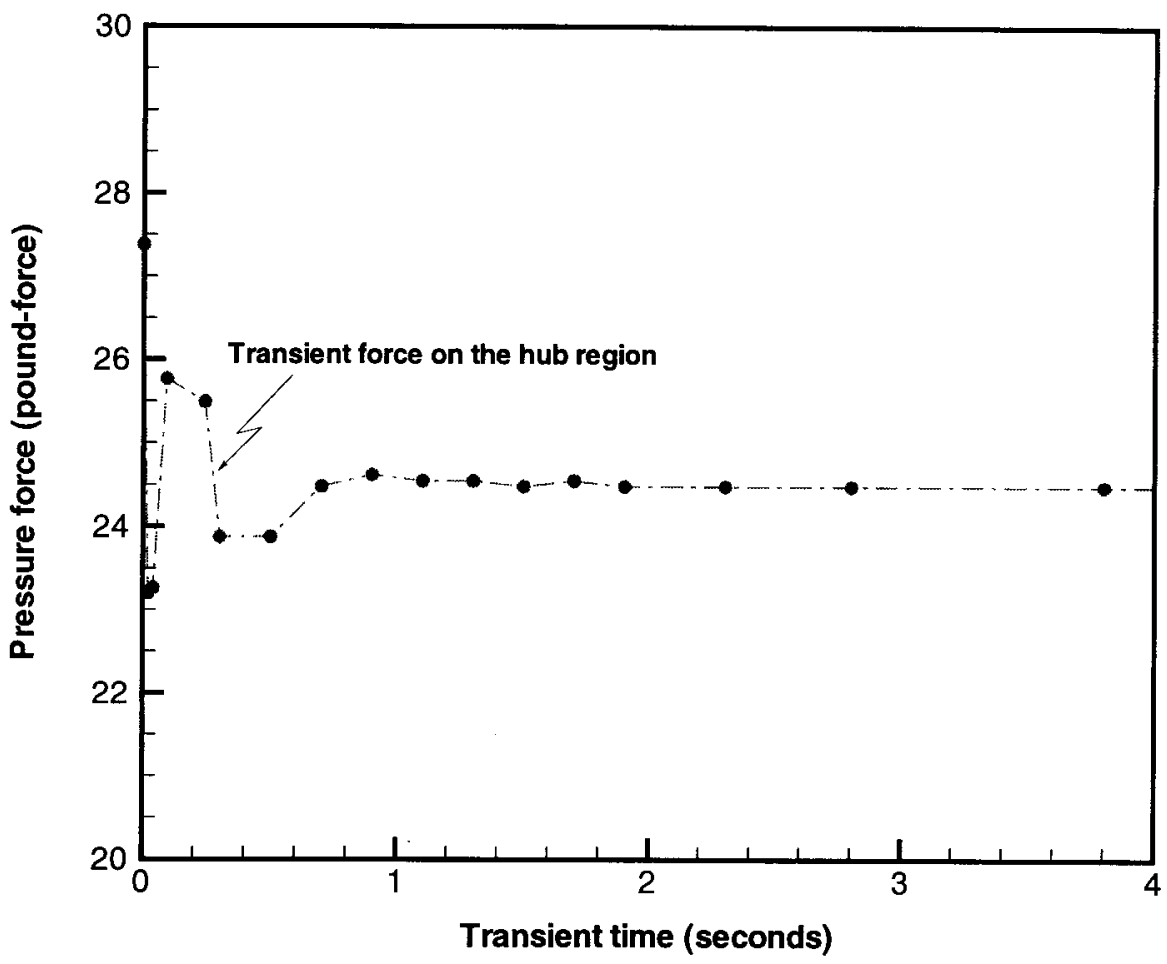

Figure 41. Transient response of pressure force for hub under $12,000 \mathrm{gpm}$ and $860 \mathrm{rpm}$ operating condition. 


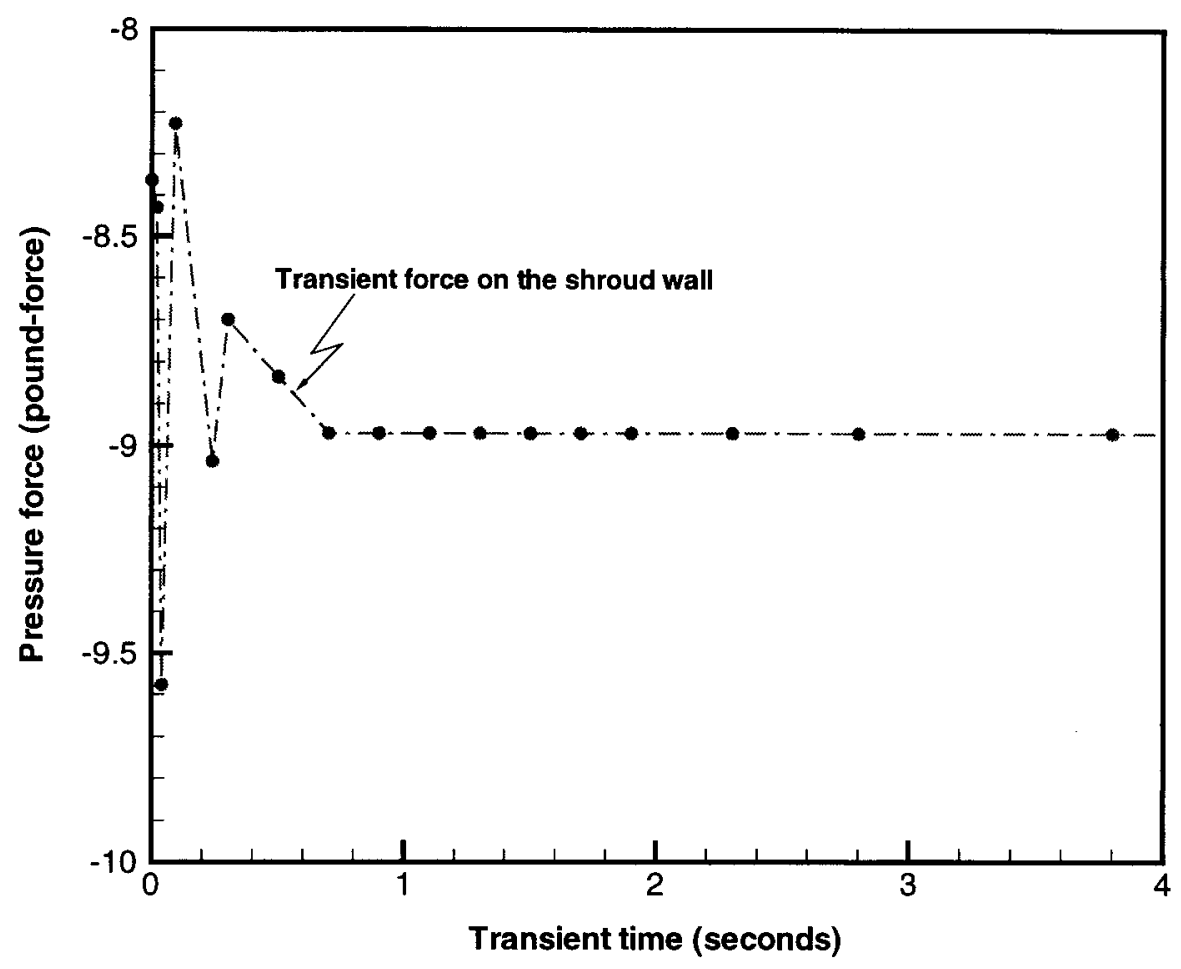

Figure 42. Transient response of pressure force for shroud wall under 12,000 gpm and $860 \mathrm{rpm}$ operating condition. 
Report:

Date:

Page:

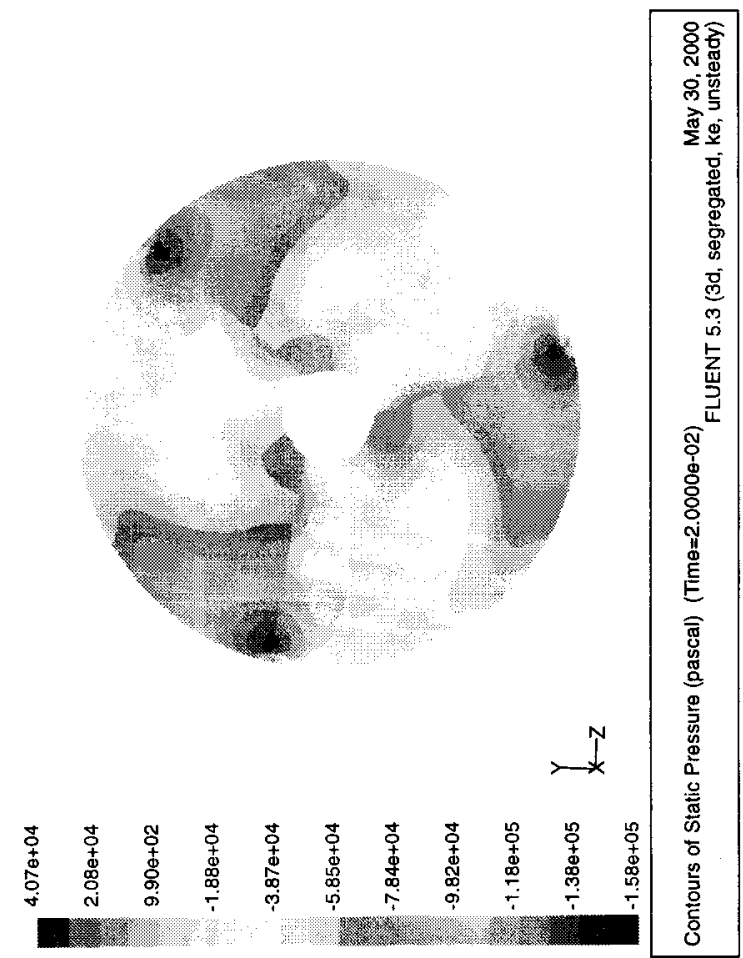

(transient time $=0.02 \mathrm{sec}$ )

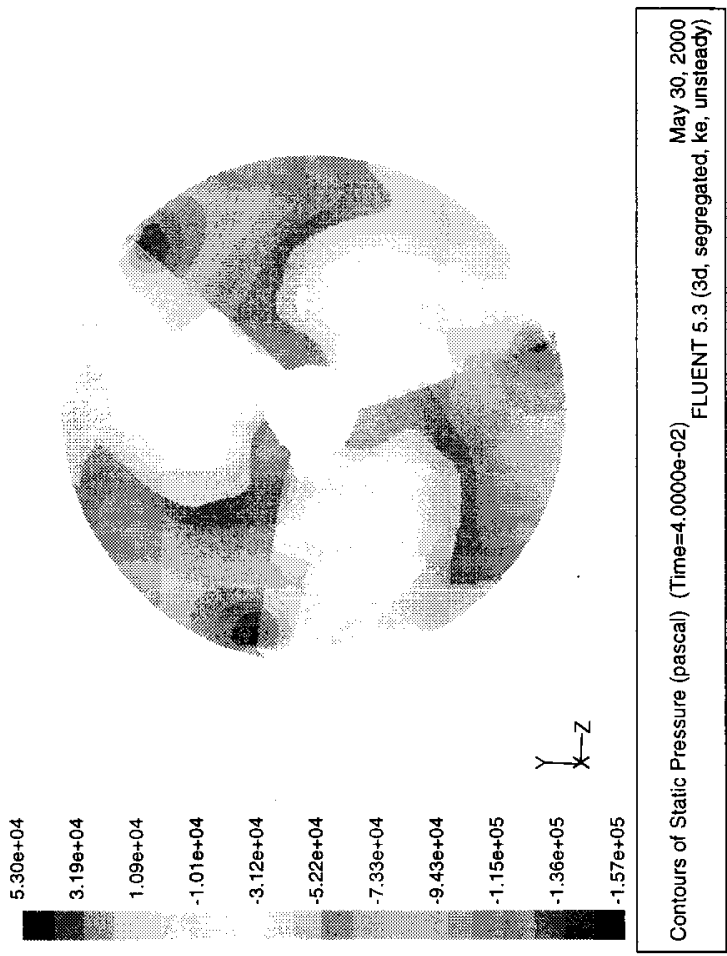

(transient time $=0.04 \mathrm{sec}$ )

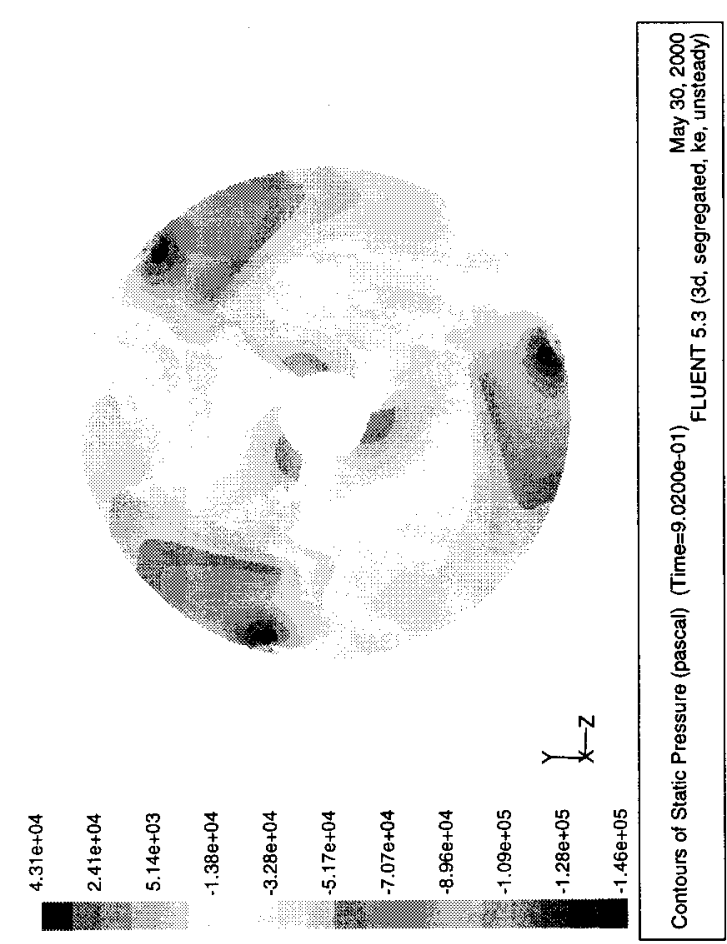

(transient time $=0.90 \mathrm{sec}$ )

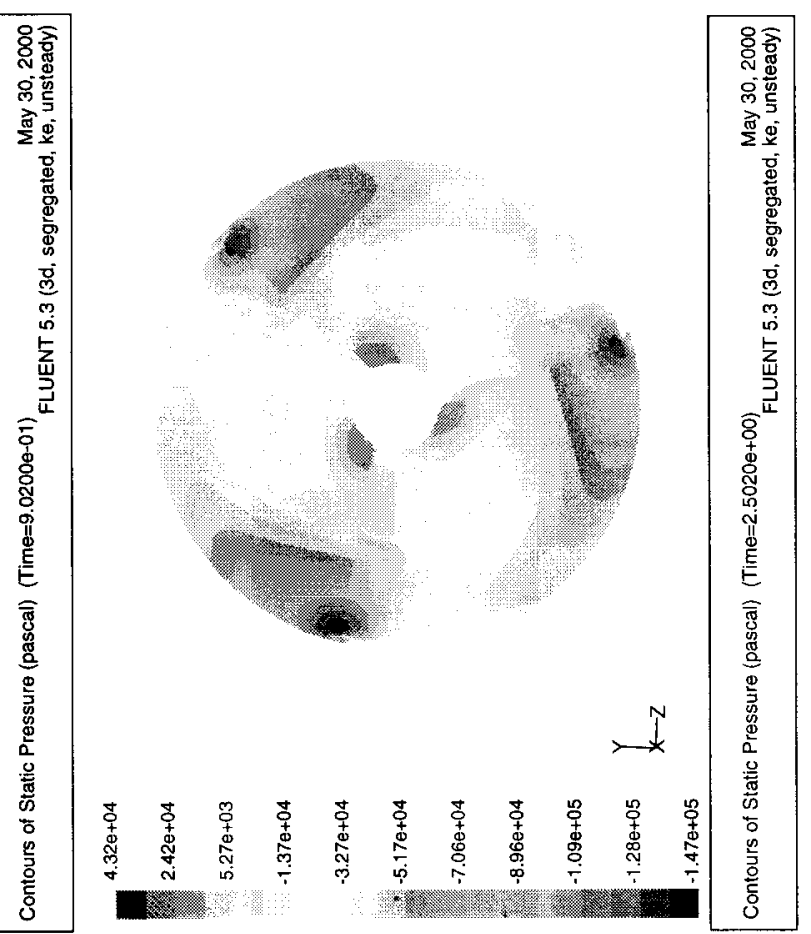

(transient time $=2.50 \mathrm{sec}$ )

Figure 43. Transient response of static pressure at leading-tip edge plane of the propeller. 


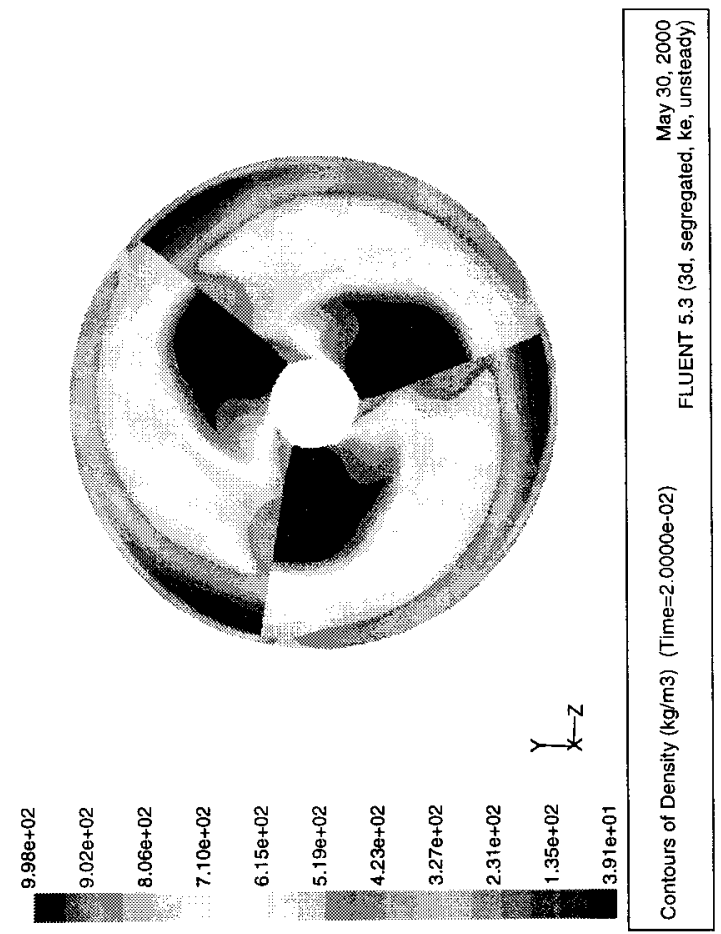

(transient time $=0.02 \mathrm{sec}$ )

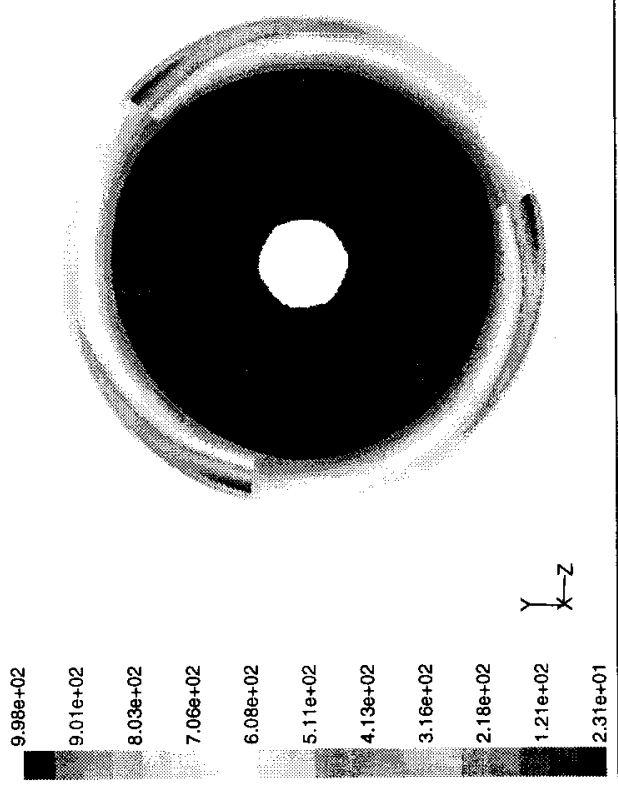

(transient time $=0.90 \mathrm{sec}$ )

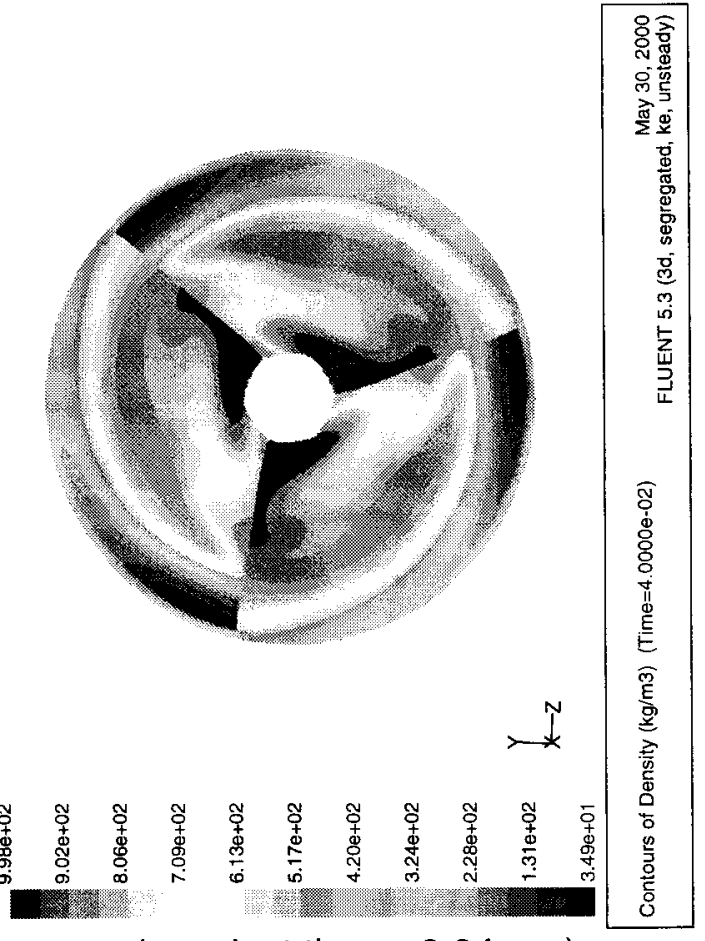

(transient time $=0.04 \mathrm{sec}$ )

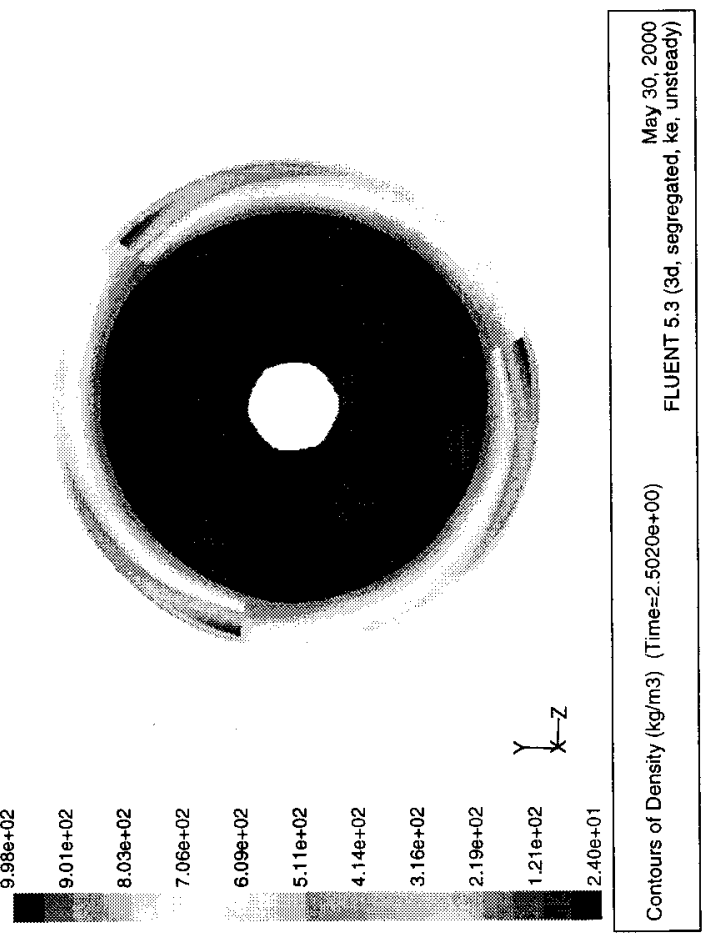

(transient time $=2.50 \mathrm{sec}$ )

Figure 44. Transient response of fluid density at leading edge plane of the propeller tip. 


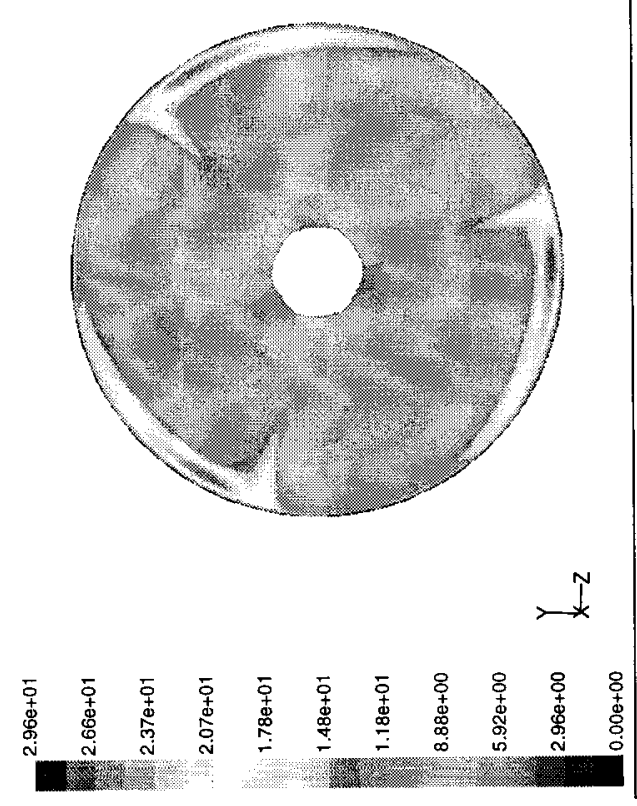

(transient time $=0.02 \mathrm{sec}$ )

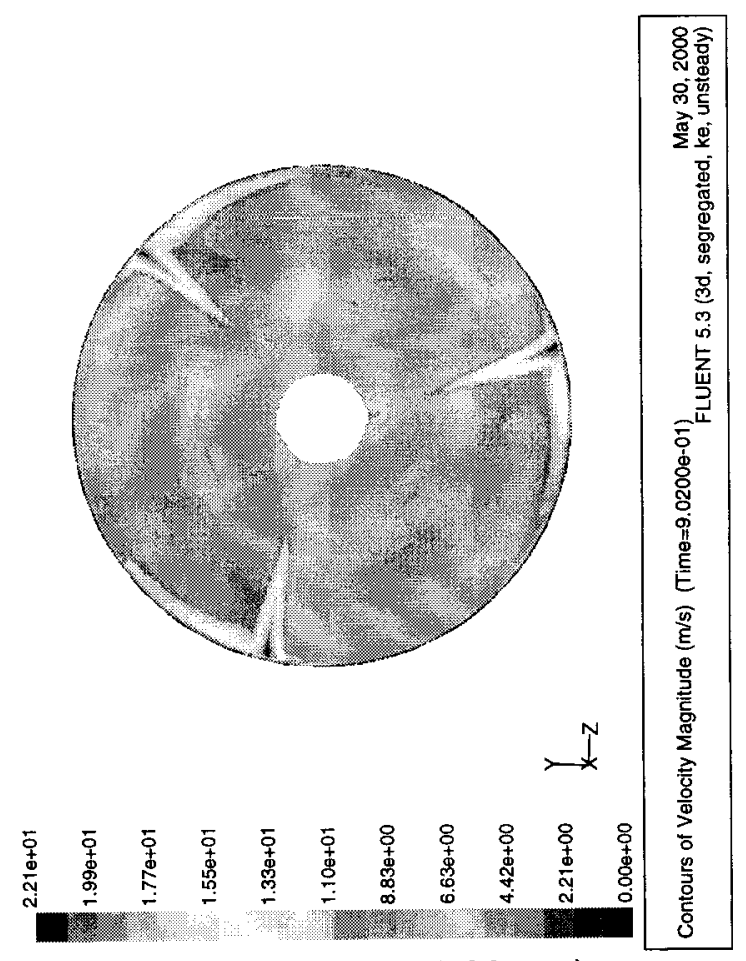

(transient time $=0.90 \mathrm{sec}$ )
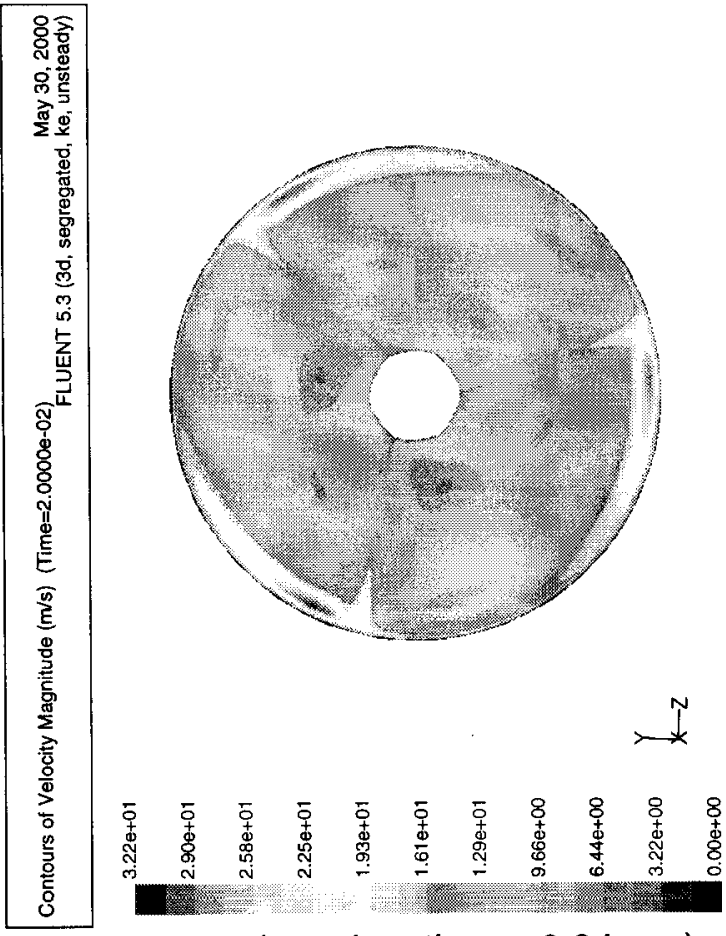

(transient time $=0.04 \mathrm{sec}$ )

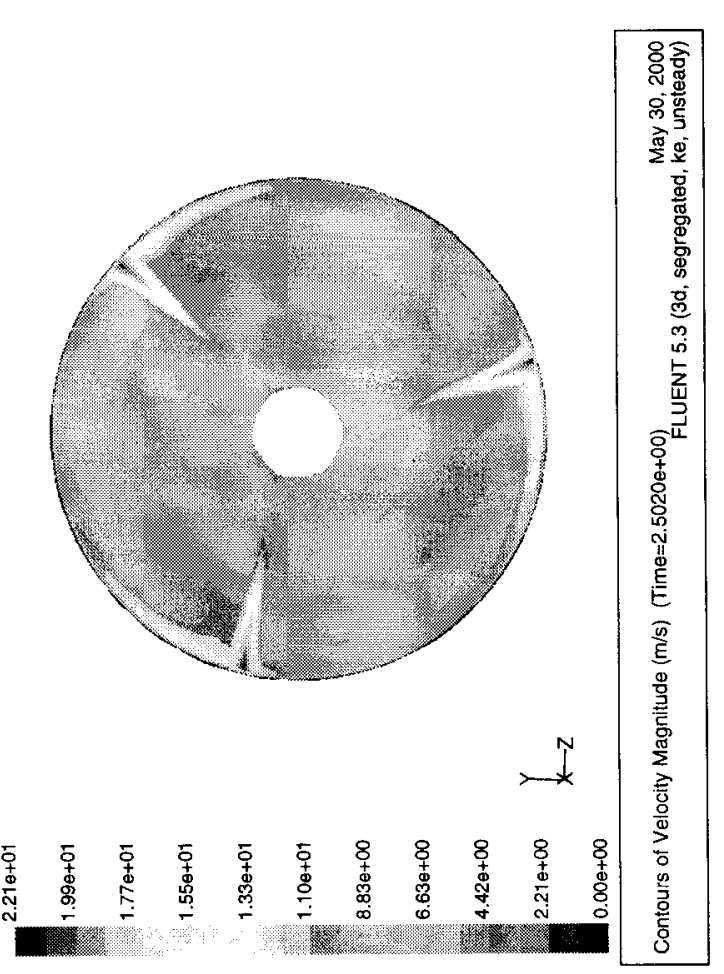

(transient time $=2.50 \mathrm{sec}$ )

Figure 45. Transient response of fluid velocity at leading edge plane of the propeller tip. 


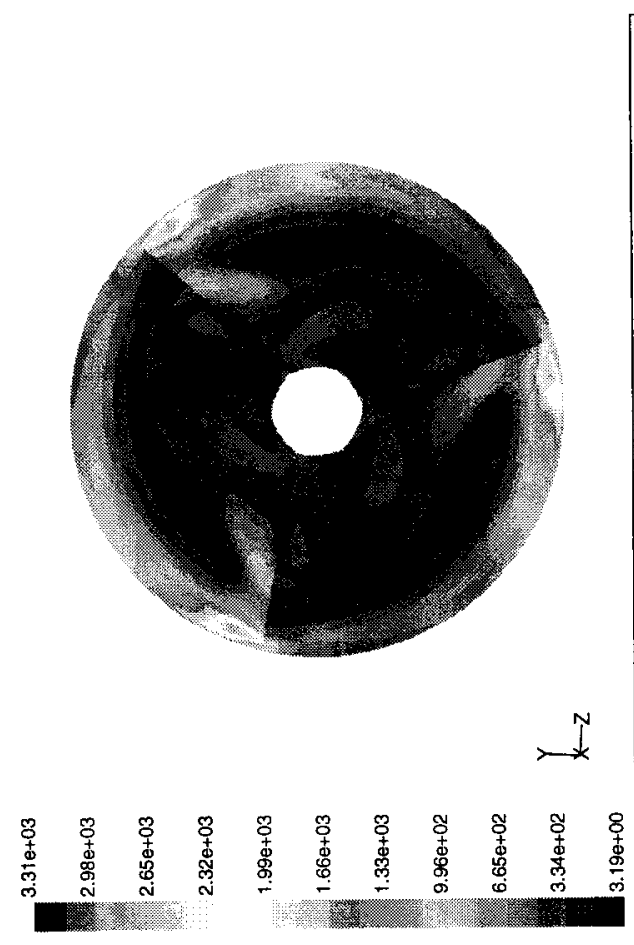

(transient time $=0.02 \mathrm{sec}$ )

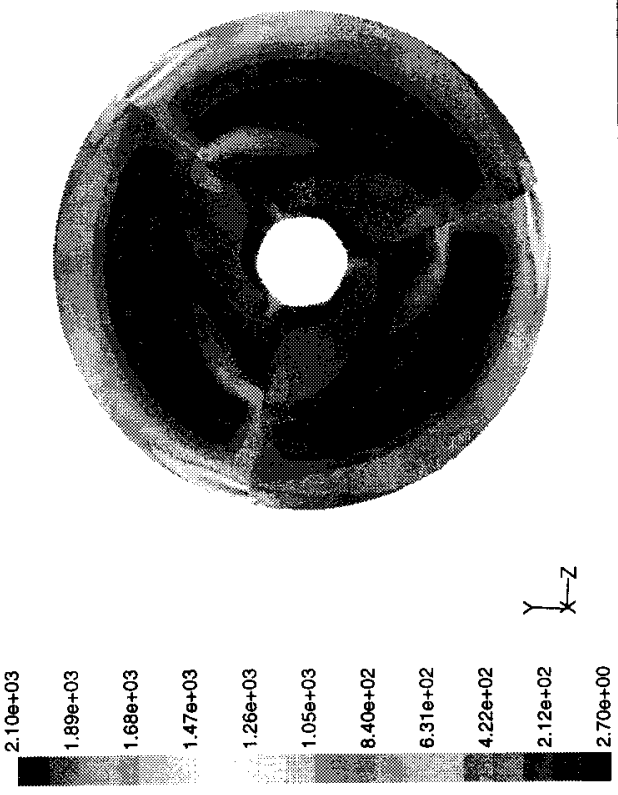

(transient time $=0.90 \mathrm{sec}$ )

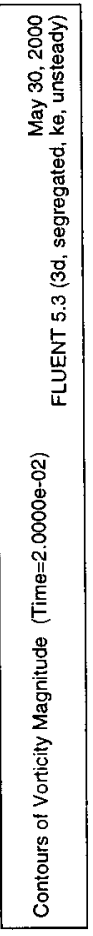

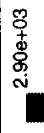

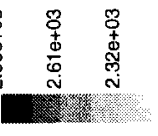

(transient time $=0.04 \mathrm{sec}$ )
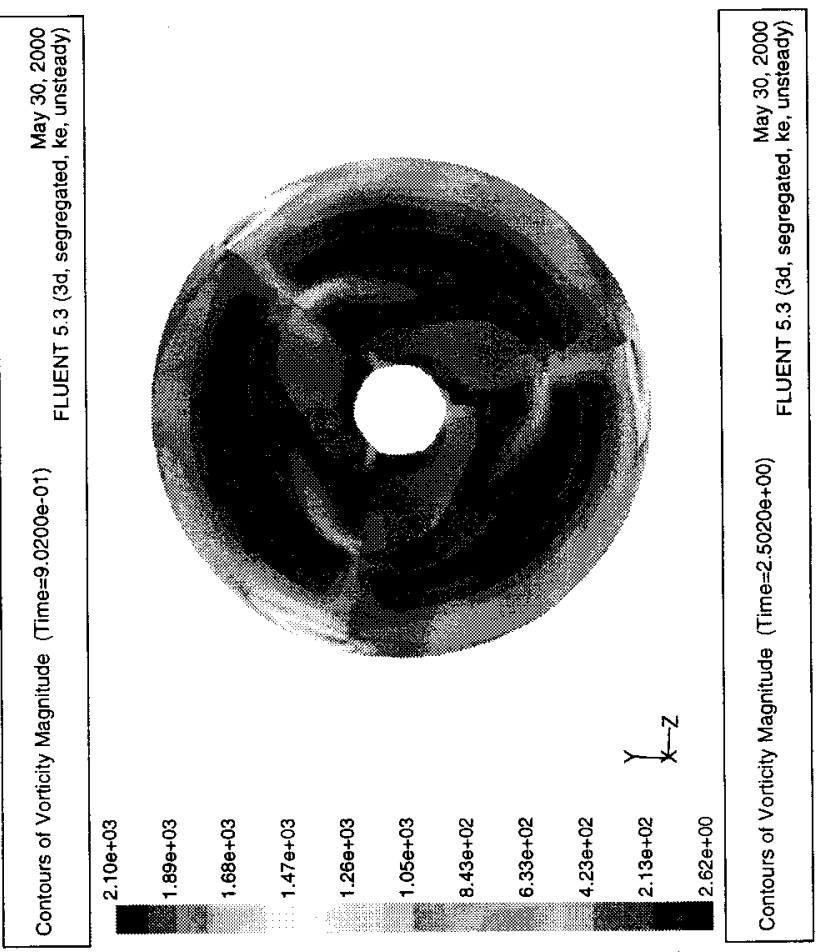

(transient time $=2.50 \mathrm{sec}$ )

Figure 46. Transient response of fluid vorticity at leading edge plane of the propeller tip. 


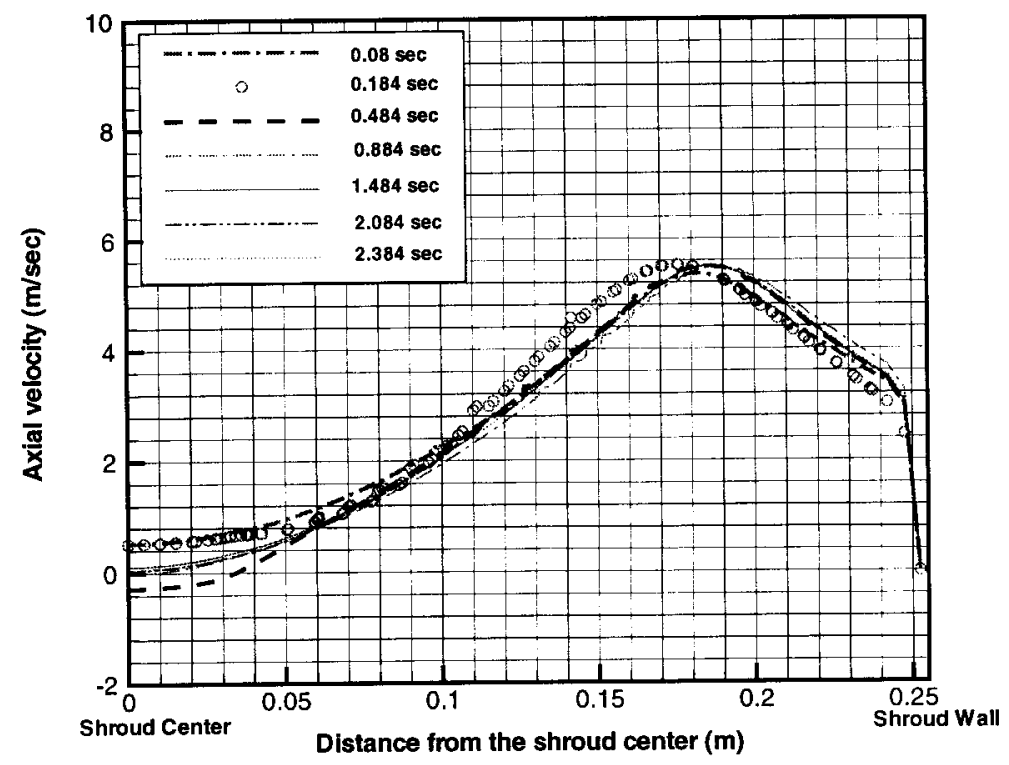

Figure 47. Transient velocity distributions at the exit of the shroud for $700 \mathrm{rpm}$ pump speed and $12000 \mathrm{gpm}$ flow condition.

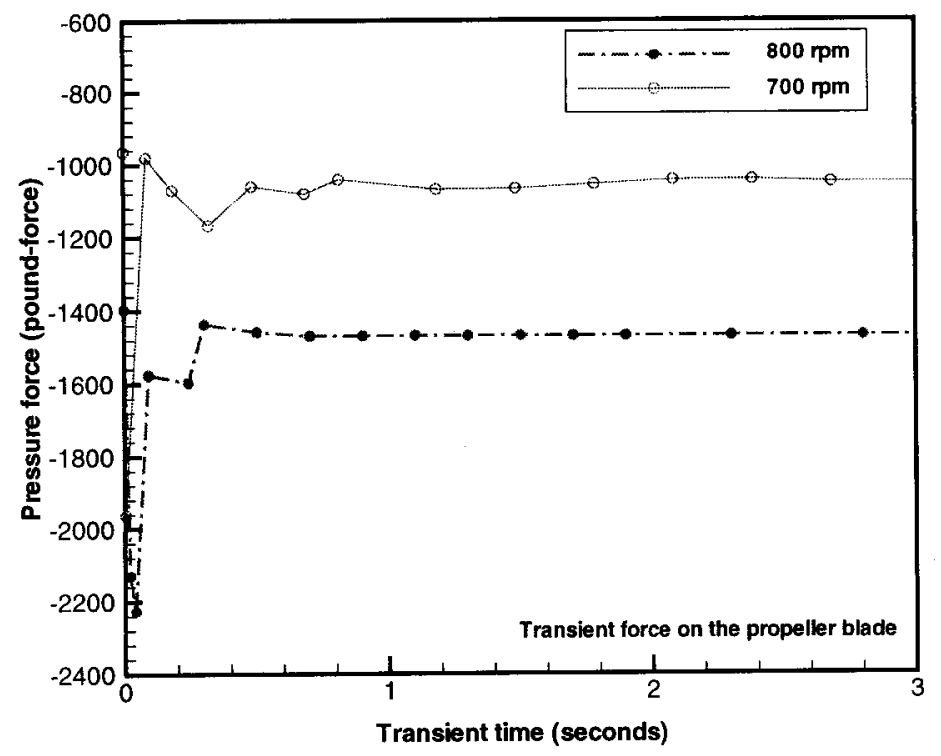

Figure 48. Comparison of transient total pressure force for the $700 \mathrm{rpm}$ propeller blades with that of the $860 \mathrm{rpm}$ mixer for $12000 \mathrm{gpm}$ flow condition. 


\section{Conclusions}

The present analysis took two modeling approaches with a Computational Fluid Dynamics (CFD) method. They are steady-state pump model and transient cavitation model considering density change effect due to local cavitation. The computations were performed for a three-dimensional detailed model of the FLYGT mixer with the prototypic propeller shape. The models mainly include propeller region, inlet region upstream of the propeller, and downstream shroud region as a computational domain. The general characteristics of the propeller flow including the blade-to-blade rotational flow, cavitation with tip vortex, and flow pattern at shroud exit were well predicted by the present CFD method using computational domain of one-third FLYGT mixer for better computational efficiency.

From the analysis results for the three-dimensional models of the Tank 19 FLYGT mixer, the following conclusions are drawn:

1. Nominal operating conditions were measured by the pitot-tube test data (Adkins, 2000). Comparisons with calculated results showed that nominal pump flow conditions were about $9000 \mathrm{gpm}$, and pump flow distribution was not sensitive to rotational speed of the mixer (600 to $860 \mathrm{rpm}$ ), which is consistent with the test data.

2. From the results of the present detailed model and the previous wall jet model, the pump with a 20" long modified shroud showed reasonable flow performance at the exit of the shroud in terms of backflow prevention and mixing capability.

3. The detailed steady-state model results showed that load for each of the three major component (propeller blade, hub and shroud) of the mixer increased as speed of the mixer increased. It was noted that cavitation occurred near the leading-tip of the pump blade and the degree of cavitation increased rapidly with pump speed increase.

4. The steady-state results show that cavitation doesn't occur for $9,000 \mathrm{gpm}$ flowrate when the pump speed is lower than $500 \mathrm{rpm}$. This is consistent with the original blade design of the FLYGT mixer to avoid the cavitation. The original design speed was 440 rpm.

5. From the sensitivity study, it was noted that the pump speed was a sensitive parameter for the load for each pump component, but it was not sensitive for the pump flow performance. The propeller blade has the largest load among the three components of the FLYGT mixer under a wide range of pump operating conditions.

6. The transient results showed that transient pump behaviors such as change of fluid density and loadings of mixer were developed to steady-state conditions within about 1 second, corresponding to about 40 cycles of the propeller blade after the initiation of the pump cavitation. In addition, a low density region was established near the peripheral region adjacent to the shroud wall because all bubbles generated as a result of cavitation migrated to the high-velocity fluid region near the tip of the blade. 


\section{Recommendations}

From the detailed model and the previous two-dimensional wall jet model, it was noted that the pump speed could be decreased without reducing the flow performance of the mixer under the current 20" single-piece machined shroud. From the present steady-state results, it is recommended that the pump speed decrease from $860 \mathrm{rpm}$ down to about $500 \mathrm{rpm}$ when cavitation needs to be avoided for better pump performance.

The present model used azimuthal symmetrical assumption at the inlet region. One third of the mixer was modeled by using symmetrical planes at both-end sides of the model for computational efficiency. Thus, the present model can not predict non-uniform inlet flow condition in an azimuthal direction. In reality, non-uniform water flow and air entrained by a vortex at the inlet region may cause periodical choking before entering the propeller region.

It is recommended that the current $120^{\circ}$ symmetry, single-blade model be extended to a three-blade mixer with the tank bottom and free liquid surface (about $4 \mathrm{ft}$ level) included in the full three-dimensional computation domain. Thus, boundary conditions at the pump inlet for the CFD model would be closer to the real situation, which is non-uniform flow distribution at the screen inlet due to the flow blockage and due to the presence of the tank bottom. This change would allow us to:

1. Remove the fixed flow boundary condition at the pump inlet.

2. Analyze pump performance as a function of operating speed.

3. Determine the optimum operating point for the existing propeller shape.

This model could also simulate three-dimensional axial flow dissipation after the shroud exit above the tank bottom to assess mixing performance. The current two-dimensional jet model has been used to estimate this performance, but it can not capture the physics of three-dimensional swirling phenomena. 


\section{References}

1. Adkins, B., "Flygt Mixer Design Optimization Test Summary", WSRC Memorandum, HLW-STE-2000-00196, May 8, 2000.

2. Davis, M. R. and Winarto, H., "Jet Diffusion From a Circular Nozzle Above a Solid Plane”, Journal of Fluid Mechanics, Vol. 101, pp. 201 - 221, 1980.

3. FLUENT User's Guide, V.5.0, Fluent Incorporated, Lebanon, NH, January 2000.

4. Hsiao, C. and Pauley, L. L., "Numerical Computation of Tip Vortex Flow Generated by Marine Propeller", Transactions of the ASME, Journal of Fluid Engineering, Vol. 121, pp. 638-645, September 1999.

5. Knapp, R. T., Daily, J. W., and Hammitt, F. G., Cavitation, McGraw-Hill Book Company, New York, 1970.

6. Kubota, A., Kato, H., and Yamaguchi, H., "A New Modeling of Cavitating Flows: A Numerical Study of Unsteady Cavitation on a Hydrofoil Section", Journal of Fluid Mechanics, Vol. 240, pp. 59-96, 1992.

7. Lee, S. Y. and Dimenna, R. A., "Validation Analysis for a Computational Fluid Dynamics calculation of a Turbulent free Jet in water" Using CFDS-FLOW3D and FLUENT (U)", WSRC-TR-95-0170, May 1995.

8. Lee, S. Y. and Dimenna, R. A., "Two-Dimensional FLYGT Mixer Shroud Model and Results", WSRC-TR-2000-00106, May 2000.

9. Poirier, M. R. and Rodwell, P. O., "Phase C Flygt Mixer Test Results (U)", WSRC-TR99-00097, March 1999.

10. Personal communications with Brannen J. Adkins, March 2000.

11. Rajaratnam, N. and Pani, B. S., "Three-Dimensional Turbulent Wall Jets", Journal of The Hydraulics Divison, Vol. 100, pp. 69 - 83, January, 1974.

12. Rohsenow, W. M. and Choi, H. Y., Heat, Mass, and Momentum transfer, Prentice-Hall, Inc., Englewood Cliff, New Jersey, 1961.

13. Schlichting, H., Boundary-Layer Theory, Translated by J. Kestin, McGraw-Hill Book Company, New York, 1979.

14. Wood, G. M., "Visual Cavitation Studies of Mixed Flow Pump Impellers", Transactions of the ASME, Journal of Basic Engineering, pp. 17-28, March 1963. 
Report: WSRC-TR-2000-00205 WESTINGHOUSE SAVANNAH RIVER COMPANY

Date: $\quad$ 04/22/02

Page: $\quad 66$ of 66

THREE-DIMENSIONAL CFD FLYGT MIXER MODEL AND RESULTS

(This Page Intentionally Left Blank) 